

Methodological developments and clinical applications of pharmacokinetic models in contrast-enhanced magnetic resonance perfusion studies

Roberto Sanz Requena

Valencia (Spain), 2010 
The research described in this thesis was carried out between July 2006 and January 2010 at the Radiology Departments of the Hospital Quirón Valencia (Valencia, Spain) and the Hospital Universitari Dr. Peset (Valencia, Spain). 


\title{
Methodological developments and clinical
}

\section{applications of pharmacokinetic models in contrast- enhanced magnetic resonance perfusion studies}

\section{Roberto Sanz Requena}

\author{
Dissertation submitted \\ in partial fulfillment of the requirements \\ for the degree of
}

\section{Doctor in Philosophy}

Supervisors:

Dr. David Moratal Pérez

Center for Biomaterials and Tissue Engineering \&

Electronic Engineering Department,

Universitat Politècnica de València, Valencia, Spain

Dr. Luis Martí-Bonmatí

Radiology Department, Hospital Quirón Valencia \&

Hospital Universitari Dr. Peset, Valencia, Spain

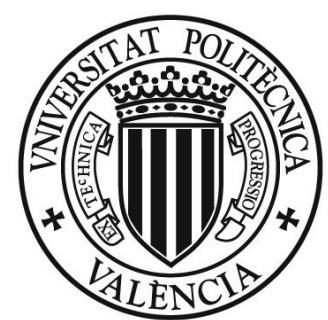

Universitat Politècnica de València 


\section{Reviewers}

Dr. Thierry Metens

Université Libre de Bruxelles

Dr. Javier Sánchez González

Philips Healthcare

Dr. Bernard Van Beers

Université Catholique de Louvain

\section{Members of the board}

Dr. Vicente Hernández García

Universitat Politècnica de València

Dr. José Vicente Manjón Herrera

Universitat Politècnica de València

Dr. Antonio Pellicer Martínez

Universitat de València

Dr. José Luis Rodrigo Pérez

Universitat de València

Dr. Javier Sánchez González

Philips Healthcare 
"De la discussió naix la llum", diuen. I l'experiència demostra que, efectivament, cadascú sol quedar-se amb les mateixes conviccions que tenia abans de discutir, però més clares.

Joan Fuster i Ortells (1922-1992) 



\section{Acknowledgements}

I can perfectly remember the day, the very moment this thesis started. Of course I still didn't know at that time, but like most relevant changes in life, it came sort of unexpected. It was spring, 2006, a young couple, a car journey on the way back from Granada to Altea, and a telephone call. Lots of stories start like that, in books, in movies, but probably few of them end up with a PhD dissertation. I can't tell what that telephone call supposed at that moment, what it meant, at least not here, so make up your own story if you wish. What concerns us now is that it was David and Luis who were behind that call, so I ended up working at Quirón Hospital, of which my aunt used to say "Quirón, cada escaló un milló". I haven't ever seen those milions, but I got the opportunity to work on a project which was at that time an incredible mixture of wonder, mistery and risk. I'm deeply grateful to my two supervisors for having opened those doors which have led me here, without their priceless help you wouldn't have this little bit of science in your hands.

After four years of tough work I've come to meet many people and I guess I owe plenty of things to all of them, but there are some souls which clearly shine with their own light, my battle mates of the underground: Àngel and Gracian, Gracian and Àngel, I was alone and they came, and it was never the same again, long life to CQV!; the radiologists, who have helped me to learn how beautiful we are inside: Salva and Juani (the "sosios"), Cristina, Vicent, Toni, Estanis, Rosario, Enrique and Elena (please, don't pay much attention to the order!); the nurses and technicians, to whom I've had to explain once and again why the hell we sometimes annoy them with strange MR sequences: Noelia, Yolanda, Juani, Eva, Ángel, Susi, Majosé, Pedro, Rubén, Sandra, Míriam, Saray and our "super", 
Chelo; our "admin" staff, especially to Dani, with whom I've spent endless afternoons working half-silent and half-listening to all those great classics; the people at Dr. Peset Hospital: Ana, Jose, Salud, Carmen(s) and Jose Luis; and the ones who have been coming and going or passing by: Javier, who should be living in Valencia instead of Madrid, Sergio, Juan, Natalia, Fabián and Clara, I hope our roads meet again in the future. I also thank Dr. Thierry Metens and Dr. Bernard Van Beers for their wonderful predisposition and rewarding comments. Although they know very little about magnetic resonance or pharmacokinetics, my parents and my brother have known how to give me their unconditional support through all these years "away from home", for showing me the value of work, honesty and humbleness. It doesn't matter how much science we make, how high we rise or how many rivers we cross, in the end our origins will always remain within a mother's womb.

Finally, and therefore most important of all, the person who started this journey with me, in that car, that spring of 2006, on our way back from Granada to Altea. Sometimes it's difficult to know for whom we do the things we do, when we mix one and two, and the 'me' becomes the 'you'. After walking so many long and winding roads you have showed me that there's always somebody waiting for me in the end, no matter how tired, no matter how late, we always make the time for one of our long walks in the bulevar. To you, I owe it all.

R. 


\section{Contents}

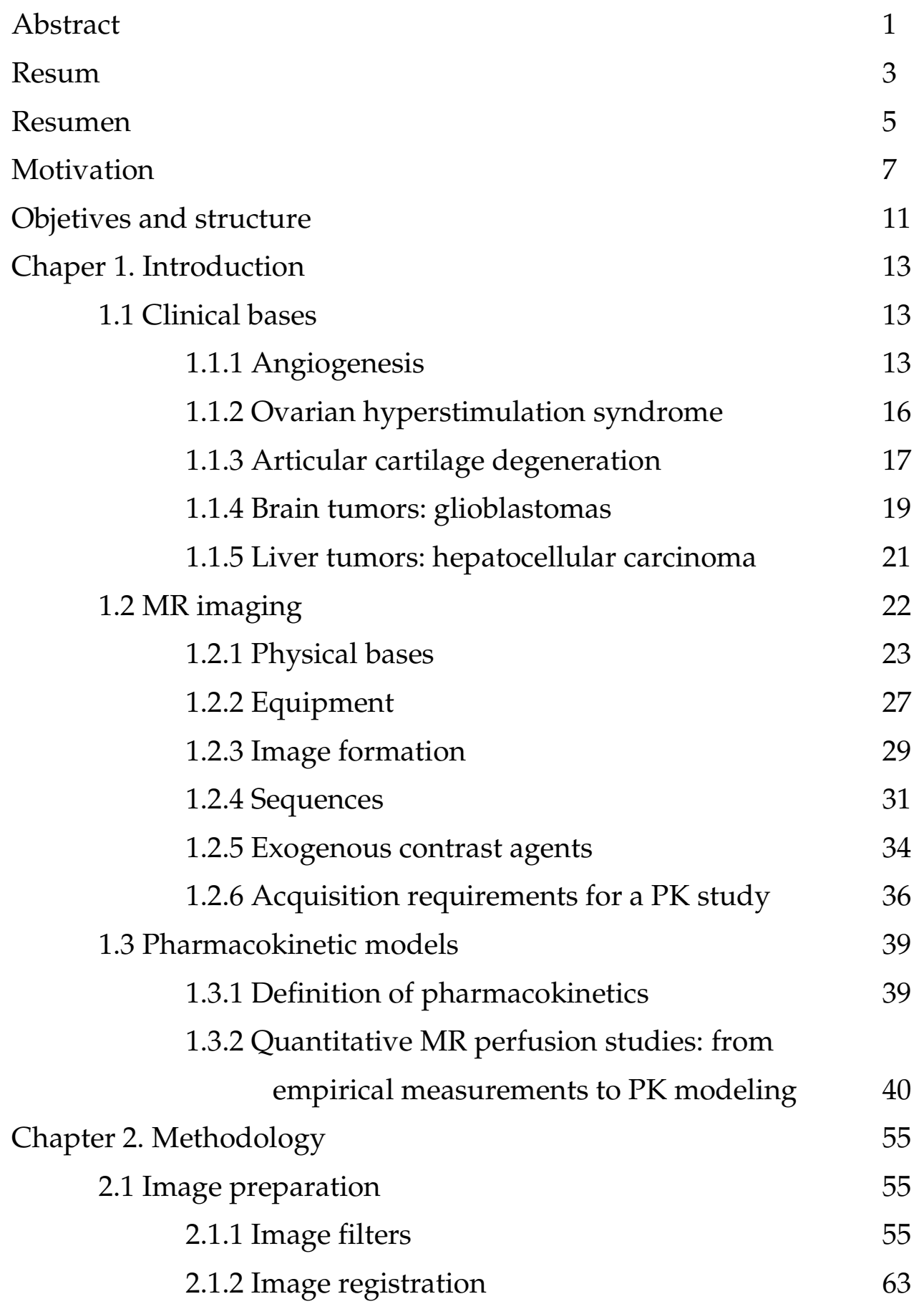


2.2 Application of PK modeling 70

$\begin{array}{ll}\text { 2.2.1 Study cases } & 70\end{array}$

2.2.2 Curve fitting 92

2.2.3 New developments 93

$\begin{array}{ll}\text { Chapter 3. Results and discussion } & 109\end{array}$

3.1 Evaluation of ovarian hyperstimulation syndrome 109

3.2 Assessment of cartilage degeneration in $1.5 \mathrm{~T} \quad 112$

3.3 Assessment of cartilage degeneration in 3.0 T 115

3.4 Evaluation of glucosamine sulfate for the oteoarthritis treatment $\quad 117$

3.5 Characterization of grade-IV astrocytomas 123

3.6 Magnetic field strength influence 127

3.7 Assessment of the arterial contribution in liver carcinomas $\quad 129$

3.8 Assessment of region heterogeneities in hepatocarcinomas 135

$\begin{array}{ll}\text { Chapter 4. Future work } & 137\end{array}$

4.1 Arterial input function 137

4.2 PK modeling of the kidneys 138

4.3 Multivariate image analysis 138

4.4 Analysis of curve fitting results 139

4.5 Methodological standardization 139

4.6 Comparison of DCE-MRI PK modeling with

IVIM and ASL 140

4.7 Development of a post-processing integrated clinical tool 141

Chapter 5. Conclusions 143

References 147

$\begin{array}{ll}\text { Publications } & 159\end{array}$

$\begin{array}{ll}\text { Appendix } & 165\end{array}$ 


\section{Abstract}

Angiogenesis and neovascularization are biological processes which take place in tissues and that are associated to an increase of the demands in oxygen and nutrients. In healthy adults these processes hardly ever occur. However, in disease conditions, such as inflammations or tumor developments, the VEGF (vascular endothelial growth factor), the signaling protein causing angiogenesis, is highly expressed. Under these circumstances new vessels and capillaries are rapidly formed. This new vasculature network is chaotic and has no normal structure, especially in the case of tumors.

The quantification of the angiogenesis is essential in order to assess the degree of tumor aggressiveness and the effectiveness of treatments. It is necessary to develop reliable and reproducible tools which are sensitive to early changes so that more individualized treatments can be used. In this sense, the pharmacokinetic modeling of perfusion magnetic resonance (MR) images is a valuable tool for the evaluation of the tissue microvascular properties, as it allows calculating parameters such as the capillary permeability, the extraction rate, the interstitial volume and the vascular volume. These models have been extensively used for the analysis of breast, liver and brain tumors; and they have also been proposed as accurate imaging biomarkers, both for the evaluation of a disease and a treatment.

In this thesis, new methodological developments and clinical applications are proposed. Regarding methodological developments, firstly a new model approached is proposed to assess arterial vascularization, based on the introduction an arterial index which quantifies the degree of arterial vascularization of any tissue in comparison to a reference arterial enhancement curve. Secondly, a temporal filter is proposed in order to improve the quality of 
the enhancement curves. Finally, a set of visualization and statistical tools is also introduced in order to improve the analysis of the results.

Regarding clinical applications, the pharmacokinetic models are implemented and applied in novel scenarios, such as the treatment of the ovarian hyperstimulation syndrome, the assessment of cartilage degeneration and the treatment of osteoarthritis. Then, further applications on the assessment of the arterial contribution of the hepatocellular carcinoma and the multivariate analysis of grade-IV astrocytomas are presented. Finally, the influence of the magnetic field strength on the pharmacokinetic parameters is also assessed.

The proposed developments and clinical applications have shown good results, as they improved the amount of information and the accuracy of the pharmacokinetic models, providing further knowledge for a better assessment of both disease and treatment effects. The new clinical applications have demonstrated the potential and the effectiveness of the pharmacokinetic parameters as imaging biomarkers extracted from perfusion MR images. 


\section{Resum}

L'angiogènesi i la neovascularització son processos biològics que tenen lloc als teixits i que estan associats a l'augment de les demandes d'oxigen i nutrients. En adults normals aquestos processos no solen ocórrer. Tanmateix, en condicions de malaltia, com a les inflamacions o el desenvolupament de tumors, el VEGF (factor de creixement vascular endotelial, de l'anglés vascular endothelial growth factor), la proteïna senyalitzadora causant de l'angiogènesi, està fortament expressada. En aquestes circumstàncies es formen ràpidament nous vasos i capil.lars. Aquesta nova xarxa vascular és caòtica i no presenta una estructura normal, especialment en el cas dels tumors.

La quantificació de l'angiogènesi és essencial per a avaluar el grau d'agressivitat d'un tumor i la eficàcia dels tractaments. És necessari desenvolupar ferramentes fiables i reproduibles que siguen sensibles als primers canvis, la qual cosa pot permetre utilitzar tractaments més individualitzats. En aquest sentit, el modelat farmacocinètic d'imatges de perfusió per ressonància magnètica (RM) és una valuosa ferramenta per a l'avaluació de paràmetres com la permeabilitat capil.lar, el coeficient d'extracció, el volum intersticial i el volum vascular. Aquestos models s'han utilitzat de forma extensiva per a l'anàlisi dels tumors de mama, fetge i cervell; i, a més a més, s'han proposat com a biomarcadors d'imatge per a una avaluació exacta de la malaltia i els tractaments.

En aquesta tesi s'han proposat nous desenvolupaments i aplicacions clíniques. Respecte als desenvolupaments metodològics, primer s'ha proposat un nou model per a avaluar la vascularització arterial, basat en el càlcul d'un índex arterial que quantifica el grau de vascularització arterial en comparació a una curva arterial de referència. Segon, s'ha proposat un filtre temporal per a millorar la qualitat de les curves de captació. Finalment s'han introduït unes ferramentes de visualització i estadística per a millorar l'anàlisi dels resultats. 
Pel que fa a les aplicacions clíniques, els models farmacocinètics s'han implementat i s'han aplicat a nous escenaris clínics, com el tractament del síndrome d'hiperestimulació ovàrica, l'avaluació de la degeneració del cartílag i el tractament de l'artrosi. Després s'han presentat aplicacions com l'avaluació de la contribució arterial al carcinoma hepatocel.lular i l'anàlisi multivariant d'astrocitomes de grau-IV. Finalment també s'ha avaluat la influència de la intensitat del camp magnètic sobre el paràmetres farmacocinètics.

Els desenvolupaments proposats i les aplicacions clíniques han mostrat bons resultats, ja que han millorat la quantitat d'informació i l'exactitud dels models farmacocinètics, oferint més coneixement per a una millor avaluació de la malaltia i dels efectes dels tractaments. Les noves aplicacions clíniques han demostrat el potencial i l'efectivitat dels paràmetres farmacocinètics com a biomarcadors d'imatge obtinguts a partir d'imatges de perfusió de RM. 


\section{Resumen}

La angiogénesis y la neovascularización son procesos biológicos que tienen lugar en los tejidos y que están asociados al aumento de las demandas de oxígeno y nutrientes. En adultos normales estos procesos no suelen ocurrir. Sin embargo, en condiciones de enfermedad, como en inflamaciones o en el desarrollo de tumores, el VEGF (factor de crecimiento vascular endotelial, del inglés vascular endothelial growth factor), la proteína señalizadora causante de la angiogénesis, está fuertemente expresada. En estas circunstancias se forman rápidamente nuevos vasos y capilares. Esta nueva red vascular es caótica y no presenta una estructura normal, especialmente en el caso de tumores.

La cuantificación de la angiogénesis es esencial para evaluar el grado de agresividad de un tumor y la eficacia de los tratamientos. Es necesario desarrollar herramientas fiables y reproducibles que sean sensibles a cambios tempranos, lo cual puede permitir utilizar tratamientos más individualizados. En este sentido, el modelado farmacocinético de imágenes de perfusión por resonancia magnética (RM) és una valiosa herramienta para la evaluación de parámetros como la permeabilidad capilar, el coeficiente de extracción, el volumen intersticial y el volumen vascular. Estos modelos se han utilizado de forma extensiva para analizar los tumores de mama, hígado y cerebro; y, además, se han propuesto como biomarcadores de imagen para una evaluación exacta de la enfermedad y los tratamientos.

En esta tesis se han propuesto nuevos desarrollos y aplicaciones clínicas. Respecto a los desarrollos metodológicos, primero se ha propuesto un nuevo modelo para evaluar la vascularización arterial, basándose en el cálculo de un índice arterial que cuantifica el grado de vascularización arterial en comparación con una curva arterial de referencia. Segundo, se ha propuesto un filtro temporal para mejorar la calidad de las curvas de captación. Finalmente se han 
introducido unas herramientas de visualización y estadística para mejorar el análisis de los resultados.

En lo referente a las aplicaciones clínicas, los modelos farmacocinéticos se han implementado y se han aplicado a nuevos escenarios clínicos, como el tratamiento del síndorme de hiperestimulación ovárica, la evaluación de la degeneración del cartílago y el tratamiento de la artrosis. Después se han presentado aplicaciones como la evaluación de la contribución arterial en el carcinoma hepatocelular y el análisis multivariante de astrocitomas de grado-IV. Finalmente también se ha evaluado la influencia de la intensidad de campo magnético sobre los parámetros farmacocinéticos.

Los desarrollos propuestos y las aplicaciones clínicas han mostrado buenos resultados, ya que han mejorado la calidad de la información y la exactitud de los modelos farmacocinéticos, ofreciendo un mayor conocimiento para una mejor evaluación de la enfermedad y de los efectos de los tratamientos. Las nuevas aplicaciones clínicas han demostrado el potencial y la efectividad de los parámetros farmacocinéticos como biomarcadores de imagen obtenidos a partir de imágenes de perfusión por RM. 


\section{Motivation}

Classical imaging methods used for the diagnosis and follow-up of disease have been based on conventional X-rays, computerized tomography (CT), nuclear magnetic resonance (NMR) and ultrasounds. Their clinical application has been built upon the qualitative judgment of the acquired image by a radiologist. Therefore, through the visual analysis of the characteristics of a lesion (basically the information related to anatomical borders and regions), the radiologist has been able to release a diagnose about the presence and type of pathology with good efficacy. In the particular case of tumors diagnosis, the biological typification of a lesion and the assessment of its response in front of a treatment is a fundamental part of the radiologist's role. To do this, diagnostic imaging is considered as one of the best methodological tools, and sometimes even the only available one, to evaluate the tumor and its characteristics. However, although diagnostic imaging is the best means to detect and assess lesions, it is not absolutely free of error.

Thanks to the use of digital images, nowadays we can obtain relevant medical information which is not visible when the radiological images are analyzed. The evolution towards completely digital environments and the release of image post-processing platforms with quantification tools and parametric image analysis has started a new revolution in medicine. These tools can extract physiological, chemical and biological measurements from the tissues, working as virtual biopsies based on the acquisition of images which are sensitive to certain properties. These measurements behave like imaging biomarkers, which can be defined as all those characteristics that can be extracted and objectively measured from the tissues. They are indirect markers of normal or pathological processes and can be used to evaluate therapeutic intervention response $[1,2]$. 
Angiogenesis processes associated to the tumors take place when the tumor reaches a certain size and the tissue can no longer guarantee the oxygen and nutrients supplies to keep on growing. Under these circumstances, tumor cells produce signaling proteins which stimulate the formation of new blood vessels (VEGF, vascular endothelial growth factor). These angiogenic processes have a series of characteristics which make them clearly distinct from all the vascularization which develops naturally throughout the rest of the body. Tumor angiogenesis is highly heterogeneous and it presents chaotic vascular structures. Also, the new vessels are fragile and very permeable to macromolecules, due to the existence of deep indents among the endothelial cells, the number of shunts increases and the blood flow becomes unstable [3].

The interest to visualize and measure tumor angiogenesis is not only circumscribed to the assessment of tumor growth or metastases development. Nowadays there are therapies which act as antiangiogenic factors, so that their efficacy and effectiveness can be quantified with perfusion based imaging biomarkers.

Although perfusion and angiogenesis can be studied with several imaging modalities, MR imaging is one of the most common options, due to its innocuous and non ionizing properties. To analyze tumor perfusion, investigators typically use images acquired during the administration of an intravenous contrast agent. Though the perfusion characteristics of a tissue are often studied through the subjective observation of its enhancement curves, there are a number of quantitative parameters which can be derived from these curves.

The intravenous contrast agent diffuses from the capillaries into the extravascular extracellular space, providing information about perfusion, capillary permeability and interstitial space volume [4]. These parameters depend on the amount of contrast that the tissue receives and can be used to describe the tissue physiology and the anomalies in the vascular processes. The capacity to analyze tumor angiogenesis allows studying therapeutic response 
and identifying non-responders before it can be evident from traditional methodology and standards (i.e., significant size change).

Contrast enhancement analysis can be performed from several methodological approximations, as it is influenced by the acquisition technique (sequence weighting, temporal resolution, injection speed or contrast media type) and the post-processing algorithms. Due to the heterogeneity of the tumor perfusion, imaging techniques must study the whole lesion with enough spatial and temporal resolutions.

Pharmacokinetic (PK) analysis of tumors is based on the direct calculation of neovascularization biomarkers (capillary permeability, washout coefficient, vascular space fraction and interstitial space fraction) through the application of compartmental fluid diffusion models [4]. The application of these models is complex and has important variability sources, both in image acquisition and in post-processing methodologies. Although there is a huge amount of studies and applications [5-21], the methodological complexity and the dependency on the model variant do not allow the reproducibility of the results among clinical centers $[5,11,22]$. However, it has been accepted that the PK analysis should be theoretically independent from both the patient and the equipment, because it works directly on contrast concentration values, instead of only signal intensity variations. This fact, together with the ability to provide a much more realistic modeling of the tissue physiological reality, make these models an important research focus for the study of tumors and their treatments. 



\section{Objectives and structure}

This thesis focuses on the development and application of PK models to magnetic resonance (MR) perfusion images, in an aim to gain insight into the microvascular changes produced by the apparition and the progression of disease.

Particularly, the following objectives are proposed:

- To adapt the generalized PK model to the study of new clinical scenarios:

- Effects of ovarian hyperstimulation syndrome treatment.

- Microvascular changes of the articular cartilage in chondromalacia and osteoarthritis.

- Microvascular changes produced by glucosamine sulfate in osteoarthritic cartilages.

- Differences between one-compartment and two-compartment models in central nervous system (CNS) gliomas.

- Assessment of perfusion changes in hepatocellular carcinomas.

- To establish the influence of magnetic field intensity on the PK parameters.

- To develop an improved methodology for the accurate assessment of the neoangiogenesis in liver tumors.

- To develop a software tool for the application of PK models in the clinical routine.

The thesis is structured as follows:

Chapter 1 is an introduction which describes all the necessary concepts to understand the thesis. Firstly, the clinical bases regarding the angiogenesis process and the clinical applications are explained. Secondly, a description of the MR imaging technique is given, focusing on the special needs of PK perfusion 
studies. And thirdly, the PK models are introduced, with a summary of their evolution, the state of the art and the current methodological problems.

Chapter 2 describes the thesis methodology. It is divided into four parts: image pre-processing steps, with the filters and the registration process that must be applied to the acquired images; PK modeling, with the proposed improvements and new developments; curve fitting algorithms; and reproducibility assessment and statistical analysis methods. In this chapter, the new developments regarding curve filtering and neovascularization modeling are also developed. In chapter 3 the results of the clinical applications and the reproducibility tests are presented: ovarian hyperstimulation syndrome, cartilage degeneration, brain and liver tumors. The discussion on the results is also presented.

Chapter 4 deals with the possible lines of future work and Chapter 5 summarizes the conclusions.

Finally, an appendix with a brief description of the developed software is also given. 
Introduction

\section{Introduction}

This chapter introduces all the concepts which are indispensable to understand the methodological and clinical applications presented in this thesis. It is divided into three parts: part one deals with all the clinical notions, comprising the generic physiological bases of the angiogenesis processes and a brief description of the diseases that are used as clinical applications; the second part describes the MR imaging technique, focusing particularly on the imaging requirements for the PK analysis; and the third part deals with the PK models, explaining their evolution, their mathematical formulation and the state of the art.

\subsection{Clinical bases}

The following subsections describe the angiogenesis process in a generic way, then it will be applied to some clinical scenarios: ovarian hyperstimulation syndrome, articular cartilage degeneration, CNS glioblastomas and hepatocellular carcinoma.

\subsubsection{Angiogenesis}

Current knowledge about oncology states that there are more than one hundred types of cancer and that there is a huge heterogeneity among them [23]. However, although they may present drastically different characteristics, the mechanisms that start a tumor and keep it growing are quite similar.

One of the mechanisms that stands in the spotlight of tumor development is angiogenesis (figure 1.1), which can be defined as the formation of new vessels either from pre-existing vasculature or from embryo cells. 


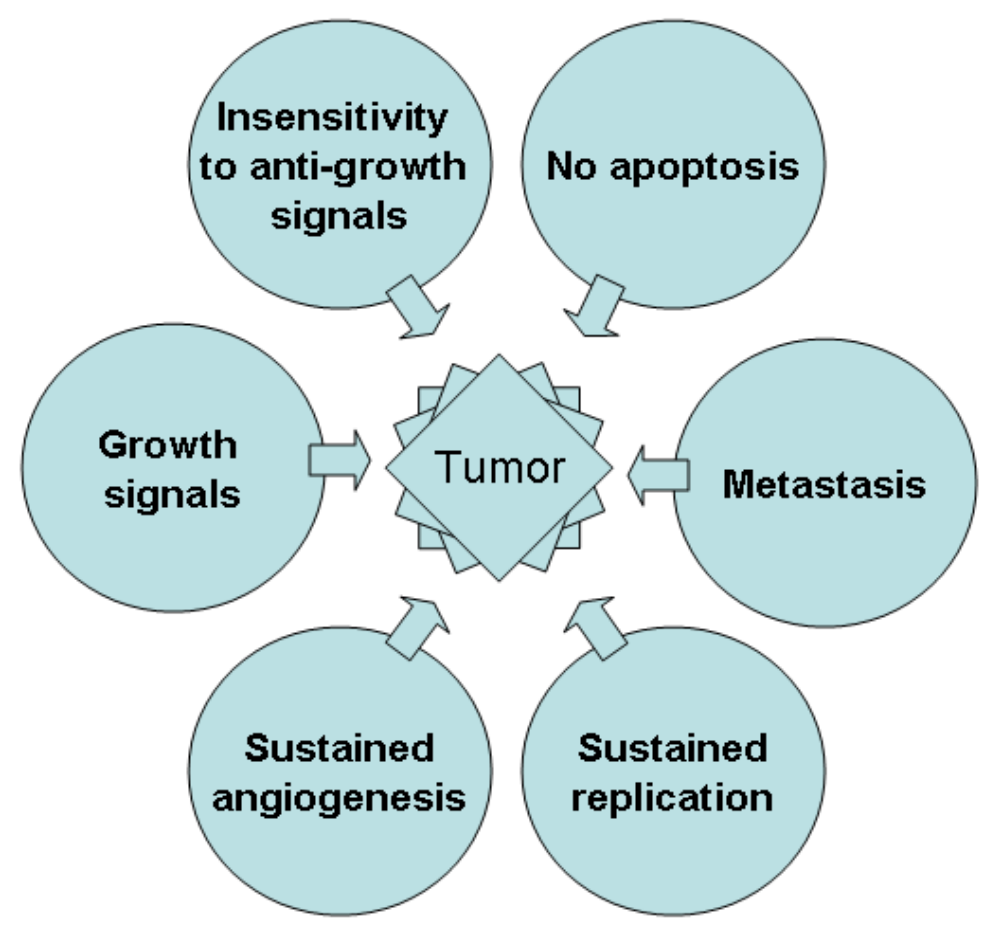

Figure 1.1. Factors involved in the origin and development of a tumor.

In healthy adults, angiogenesis seldom occurs, and it is only related to wound healing and female reproductive cycles [24]. This inhibition of angiogenesis is held by keeping a balance between angiogenic factors and inhibitors.

When a tumor is developing, at a certain step of growth (approximately $1 \mathrm{~mm}$ ) it needs an autonomous blood supply to keep on growing [25]. To achieve this, it breaks the vascular balance (angiogenic switch) and develops an overproduction of growth factors that generate new vessels out of the existing vascular network (figure 1.2). Among these growth factors, the VEGF (vascular endothelial growth factor) is the only one which is produced through the whole tumor life [26]. It has been shown to be present in most tumor types (colon, lung, breast, brain, kidneys, ovaries, prostate, etc.), keeping a strong relation with tumor development [27]. 


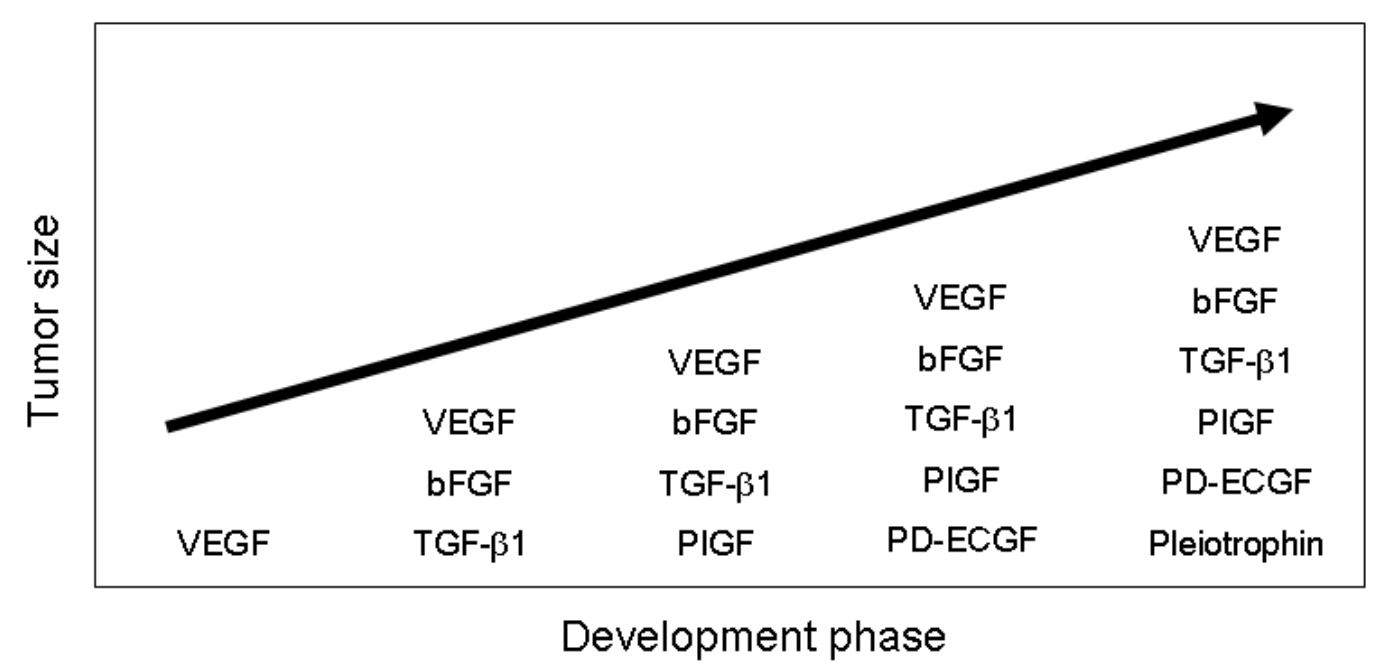

Figure 1.2. Biochemical markers produced in the tumor during its evolution.

The VEGF acts over the tumor vasculature in three ways [28]:

- Establishment of new vasculature through the recruitment of bonemarrow-derived cells that are used to set up the new vascular network [29].

- Growth of new vasculature by providing additional blood supply.

- Maintenance of the blood supply network, inhibiting apoptosis and sustaining the metabolic needs of the tumor.

Tumor vascular networks are formed by a complex and chaotic mesh of vessels and arterio-venous shunts. This produces very heterogeneous and unstable environments [30], where blood vessels have serious structural and functional abnormalities. This fact makes tumors a difficult target for a uniform delivery of drugs to the whole tumor.

Many anti-angiogenic treatments focus their mechanisms in the inhibition of the VEGF signaling pathway, both in intra- and extracellular environments [28]. However, these treatments are not always effective, due to the properties of some tumors to develop resistance to these inhibitors through mutation processes. 
When this happens, tumor progression continues through the activation of secondary pathways [28].

So far there are not yet adequate means to assess and measure tumor angiogenesis in a reproducible, direct and reliable way. Despite their limitations, these are some of most commonly used markers [31]:

- Microvessel density (MVD): MVD is determined immunohistochemically and it represents the amount of oxygen and nutritional supply of proliferating tumor cells, as well as the potential for invasion and metastasis.

- Intratumoral VEGF determination: it can be measured using immunohistochemistry, in situ hybridization, immunoassays or reversetranscriptase polymerase chain reaction. However, it is a very expensive method which is not used in clinical routine.

- Monitoring circulating VEGF: some studies conclude that the amount of VEGF in blood is related to tumor status and prognosis in most types of cancer. However, this measurement may not be useful in early disease steps, such as for screening purposes.

\subsubsection{Ovarian hyperstimulation syndrome}

Ovarian hyperstimulation syndrome (OHSS) is a supraphyisiologic secondary response to the gonadotropins stimulus in an ovulation induction (figure 1.3). It is the result of increased vascular permeability and extravasation of fluid, which also causes hemoconcentration with reduced organ perfusion [32]. Severe forms of OHSS appear in $0.5-5 \%$ of assisted reproduction technology cycles and can result in death [33]. Despite being a dangerous iatrogenic complication, its pathophysiology remains unknown and it has always been treated empirically. 


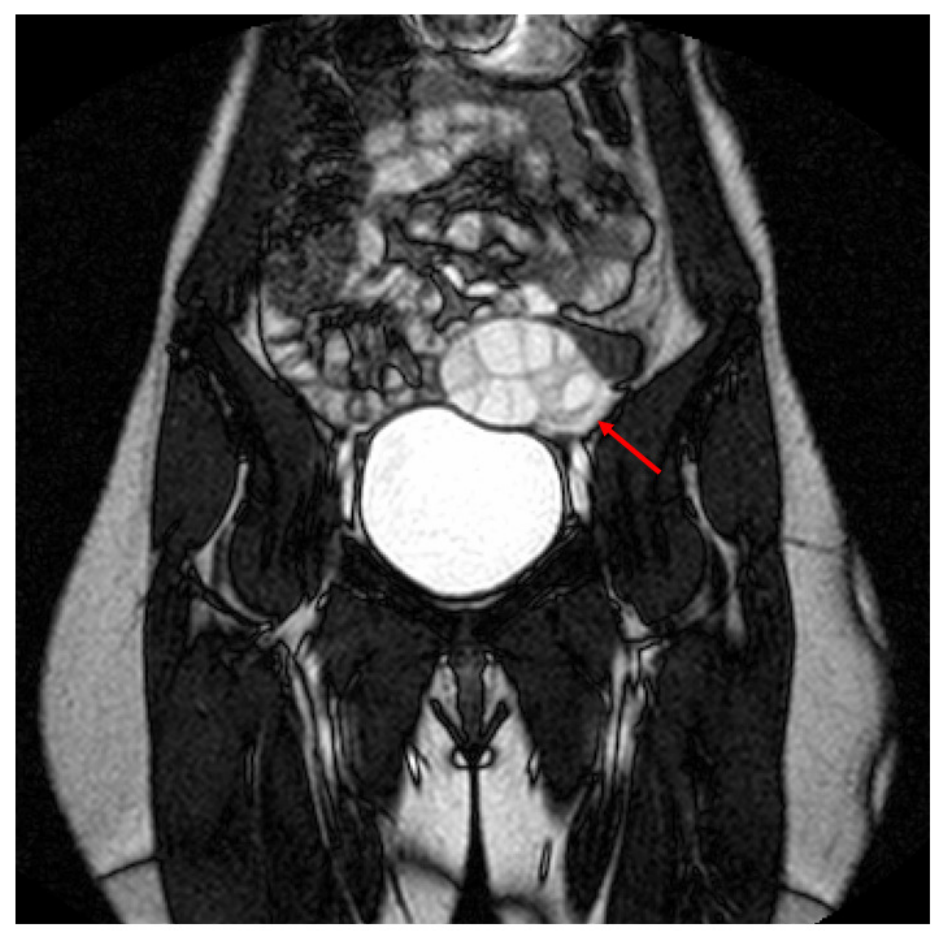

Figure 1.3. OHSS. It can be seen that the ovaries (red arrow) are clearly oversized.

OHSS develops after the administration of human chorionic gonadotropin (hCG). As hCG has no vasoactive activity, VEGF is produced as mediator of hCG-dependent ovarian angiogenesis. It is known that elevated levels of VEGF mRNA are detected in serum, plasma and peritoneal fluids in women at risk or with OHSS [34].

Dopamine and its agonists, such as cabergoline $(\mathrm{Cb} 2)$ can inactivate VEGF selectively, preventing the development of angiogenesis and permeability. Recent studies in women with OHSS have demonstrated this protective effect during controled ovarian hyperstimulation [35].

\subsubsection{Articular cartilage degeneration}

Chondromalacia and degenerative osteoarthritis are common diseases of the articular cartilage which affect the life quality of millions, impeding the 
development of a normal life [36]. Chondromalacia affects the main substance and the collagen fibers, as well as the deepest layers of the cartilage. It tends to develop towards femoropatellar osteoarthritis, where water content increases and the thickness of the collagen matrix decreases. As a consequence, the cartilage suffers mechanical overloads and tissue destruction [37].

The gold standard to assess cartilage status is arthroscopy with biopsy, an expensive and invasive technique. MR imaging allows the study of the cartilage with high detail, offering both spatial and contrast resolution.

Traditional cartilage MR imaging biomarkers have been related to the Outerbridge classification [38], where cartilage condition is stated according to a visual analysis of the images through four grades, from normal (grade 1) to frank osteoarthritis (grade 4). MR imaging cartilage analysis is usually performed by the qualitatively grading of the cartilage signal intensity (brightness in T2 weighted images is related to water content and tissue edema) and surface condition (thinness, irregularities and erosions are related to degeneration) (figure 1.4). The use of perfusion MR imaging has increased the knowledge about cartilage abnormalities, focusing on its properties of vascularization and blood supply [39].

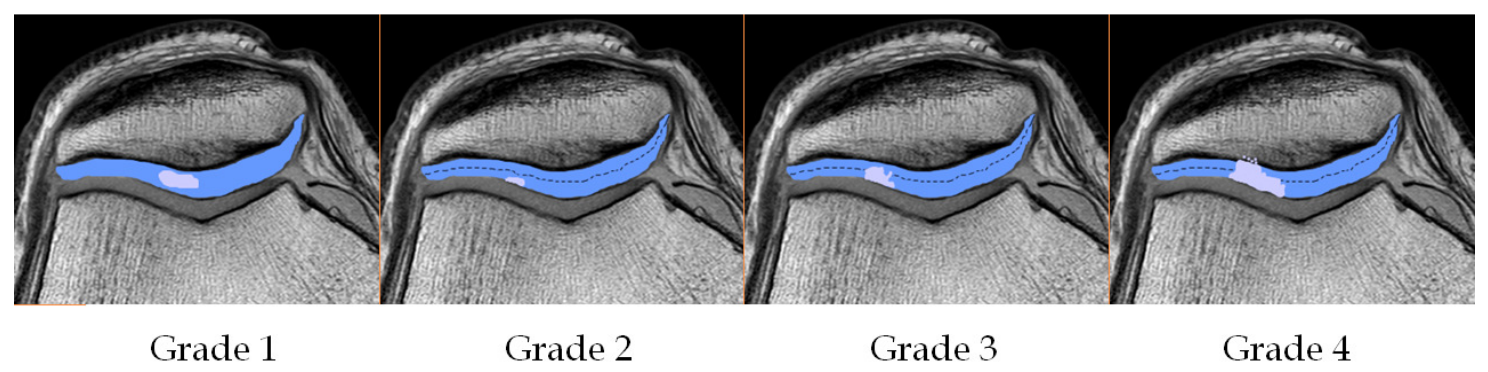

Figure 1.4. Changes in the patelar cartilage produced by the developoment of chondropathy and osteoarthritis. As degeneration progresses there is a destruction of the cartilage matrix, until the bone itself is exposed (grade 4).

Normal articular cartilage is resistant to vascular invasion. This unique property is a necessity to maintain its biomechanical properties. However, several studies 
have demonstrated that as degeneration progresses towards osteoarthritis, there is a change in the antiangiogenic cartilage properties. Recent studies have shown a positive correlation between osteoarthritis progression and the matrix expression of the VEGF [40-47]. Even in early cartilage damaging conditions, there is an overexpression of vascular mediated factors, such as the VEGF, in the chondrocytes microenvironment related to hypoxia, inflammation and excessive mechanical loading conditions [46] (figure 1.5). It seems therefore appropriate to target cartilage vascular parameters, related to VEGF expression, as an appropriate way to determine both cartilage disease status (from early changes to more advanced degeneration conditions) and the changes associated to therapy.

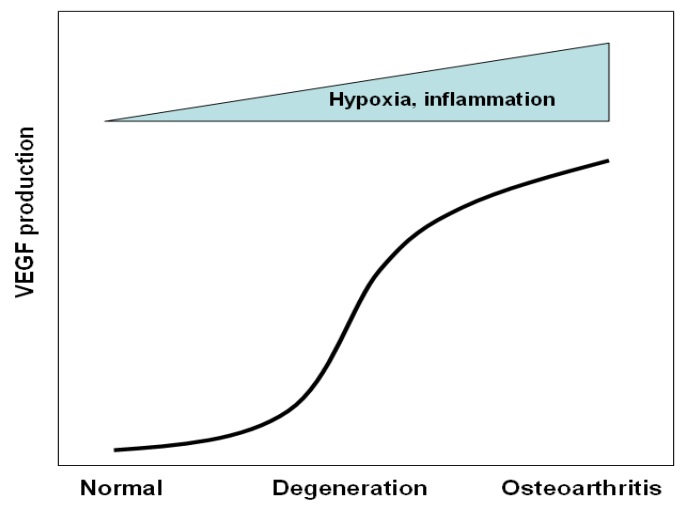

Figure 1.5. Relationship between the production of VEGF and the degeneration progression.

\subsubsection{Brain tumors: glioblastomas}

Astrocytomas are the most frequent primary brain tumors of the central nervous system. The World Health Organization classifies them as circumscribed or diffuse [48]. The diffuse astrocytomas are: grade II or low grade astrocytomas, grade III or anaplasic, and grade IV or glioblastomas. Glioblastomas constitute the biggest group and they also have the worst prognosis, with an average survival period of fifteen months [49]. They are heterogeneous tumors with a predisposition to infiltrate the brain tissue following the vascular structures and 
the white matter tracts (figure 1.6). Complete surgical resection of glioblastomas is not usually possible.

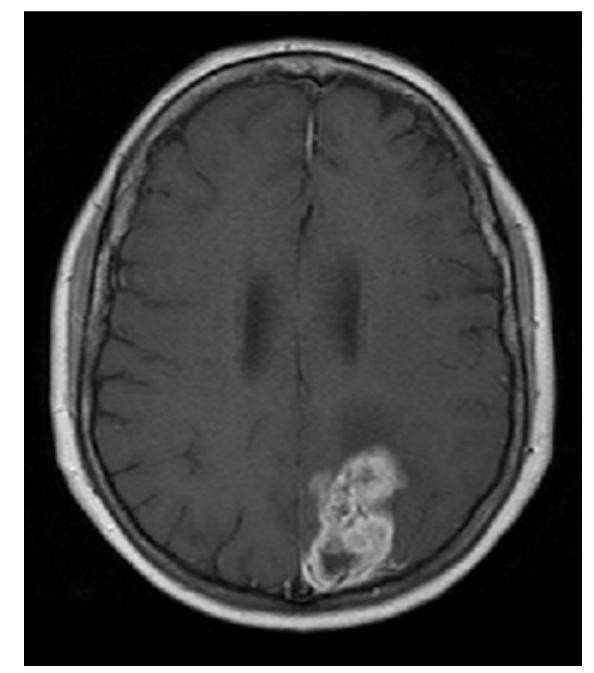

Figure 1.6. T1-weighted late enhancement image of a grade-IV astrocytoma. It can be seen that the contrast media has extravasated due to the destruction of the blood brain barrier.

The anatomopathological diagnose of glioblastomas is based on cellular atypia, presence of mitosis, vascular proliferation and necrosis. Due to their heterogeneity, the diagnose based on histological samples, obtained by biopsy or surgical resection, is not error free. Even in wide resections, histology may only be obtained out of a sample and not from the whole lesion. These sampling mistakes may lead to a wrong classification of the tumor, with the corresponding assignment of an inadequate treatment. Moreover, current tumor classifications fail when trying to predict individual tumor response in the same histological group [50]. This means that there is not a complete correlation between the tumor grade and its biological behavior.

One of the main characteristics of high grade tumors is the increment of blood vessels, a physiological parameter which indicates their biological activity and which is used as a predictive factor of bad prognosis [51]. The newly formed vessels are more tortuous and immature and they have more permeability towards macromolecules diffusion than normal brain vessels. 
Introduction

MR perfusion studies with dynamic susceptibility-contrast sequences allow quantifying angiogenesis indirectly by measuring tumor capillary permeability, a biomarker of malignancy. Moreover, this information can be obtained from the whole tumor, not only from a sample, therefore offering whole in vivo knowledge of the tumor physiology and heterogeneity.

\subsubsection{Liver tumors: hepatocellular carcinoma}

Hepatocellular carcinoma (HCC) is one of the most common malignant tumors worldwide, affecting more men than women and causing more than 600,000 deaths per year [52]. It is usually related to either a viral infection (hepatitis B or C) or cirrhosis (mainly due to alcoholism). The usual outcome is poor, as it is very difficult to remove the tumor completely after surgery (figure 1.7). Percutaneous tumor ablation techniques are used with good results in nonsurgical cases. When it cannot be completely removed, it is usually deadly within 3 to 6 months [53].

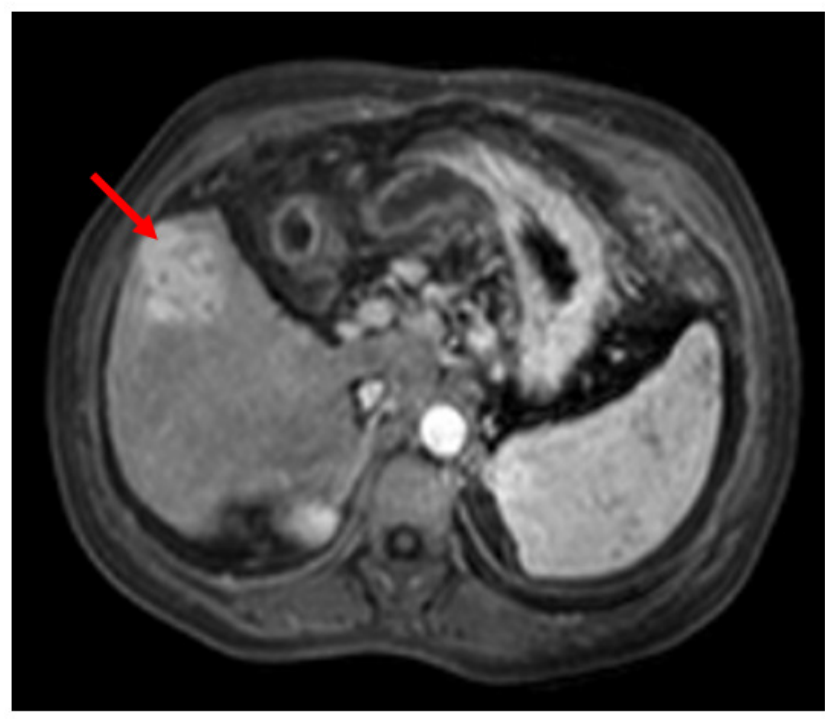

a

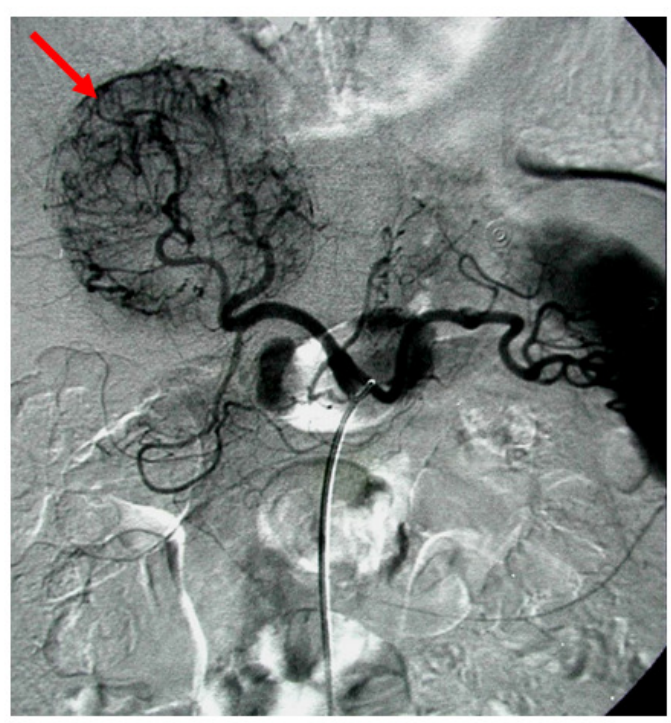

b

Figure 1.7. (a) Dynamic contrast-enhanced image of an hepatocellular carcinoma. It can be seen that the lesion has much more enhancement (it receives much more blood) than the normal liver parenchyma. (b) HCC angiography. There is a huge increase in the arterial contribution. 
The pathogenesis of most HCCs starts with chronic hepatic damage caused by the infection. During this process there is an extensive death of hepatocytes which stimulates a chronic cellular regeneration that may lead to fibrosis (cirrhosis). As the disease progresses, the healthy tissue is replaced by fibrotic tissue, which may block blood flow through the liver and thus damage the hepatic function. This damage is irreversible, although it may be considerably delayed with appropriate treatments. The high rate of cellular activity which takes place during the cirrhotic process increases the probability of mutation occurrence and the transformation of pre-tumoral hepatic lesions into HCC. HCC tends to spread forming metastasis in (intrahepatic) and out of the liver (lungs, bones and lymphatic nodes).

There are three major types of HCC:

- Expansive: it is an encapsulated tumor which grows expanding, compressing and destroying the tissue that surrounds the liver.

- Infiltrative: it is not a much defined tumor whose growing pattern is invasive.

- Multifocal: it is formed of many small tumors of similar size which are scattered through the whole liver.

\subsection{MR imaging}

MR imaging is a noninvasive tool to explore the internal anatomy and physiology of living subjects in vivo. In the nuclear MR phenomenon, atomic nuclei situated under an intense magnetic field are exposed to electromagnetic waves, which they absorb and reemit at a certain resonant frequency in the radiofrequency $(\mathrm{RF})$ range, from which the image is formed [54,55]. It is considered a safe technique, because at the intensities used in MR scanners, the RF waves have no demonstrated dangerous effects [56]. 
Introduction

\subsubsection{Physical bases}

\section{Nuclei and magnetic fields}

Nuclear MR phenomenon is caused by the fact that some nuclei with nonzero spin possess tiny magnetic moments, similar to a bar magnet. When an external magnetic field $\left(B_{0}\right)$ is applied, these magnetic moments start to rotate around its direction in a motion called precession (figure 1.8). The frequency at which the nuclei precess is called the Larmor frequency and it is proportional to the strength of $B_{0}$ and the gyromagnetic ratio of the nucleus $(\gamma)$.

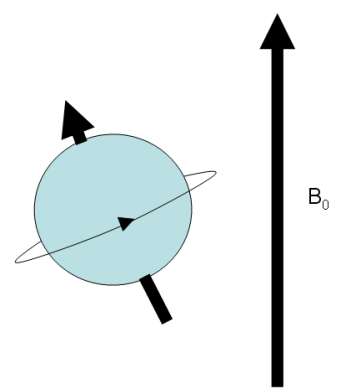

Figure 1.8. Magnetic moment of a hydrogen proton under the effect of an external magnetic field $B_{0}$. It precesses at the Larmor frequency, which is proportional to the magnetic field intensity.

In MR imaging, the most important nucleus is hydrogen, because it is the most abundant element in the body. It has a $\gamma$ of $42.58 \mathrm{MHz} / \mathrm{T}$ and, for clinical MR scanners, the Larmor frequency falls in the $\mathrm{MHz}$ range. However, hydrogen is found in many different molecular environments (water, fat, proteins, etc.). This originates small changes in the Larmor frequency, known as chemical shift.

The magnetic moment of a nucleus under a $B_{0}$, precessing at the Larmor frequency, produces oscillations in the magnetic field that can be detected with an RF coil in the transverse plane (perpendicular to $B_{0}$ ), forming an MR signal. Under equilibrium conditions ( $B_{0}$ alone), the oscillations from all the precessing nuclei are asynchronous and cancel each other, resulting in a null detectable 
signal in the transverse plane $\left(M_{x y}\right)$. However, in the longitudinal plane (parallel to $\left.B_{0}\right)$ a small non oscillatory net magnetization $\left(M_{0}\right)$ remains (figure 1.9 ).
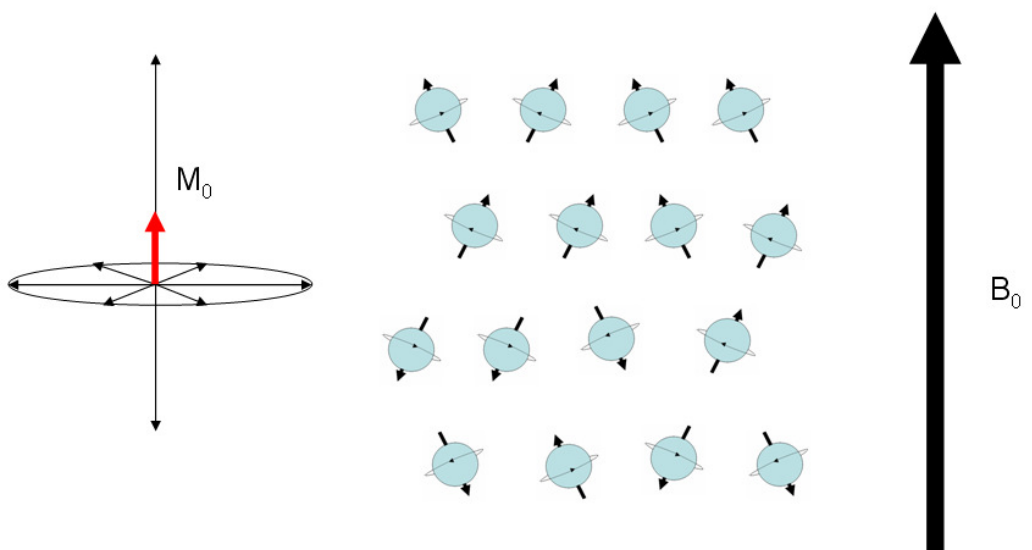

Figure 1.9. Net magnetization of a group of hydrogen protons under $B_{0}$. It can be seen that magnetization in the transversal plane is null due to global cancellation. However, there remains a net magnetization $M_{0}$ in the longitudinal plane.

\section{Excitation and detection}

The net magnetization $M_{0}$ can be tilted upon the transverse plane with the application of an external RF pulse at the Larmor frequency $\left(B_{1}\right)$. Therefore, the magnetization in the transverse plane $M_{x y}$ increases ( $M_{0}$ decreases), as the nuclei are now precessing in synchrony. When $B_{1}$ is turned off, $M_{x y}$ starts to decay due to the gradual loss of synchrony. However, during this decay it produces an oscillating magnetic field which can be detected with a receiver coil (figure 1.10).

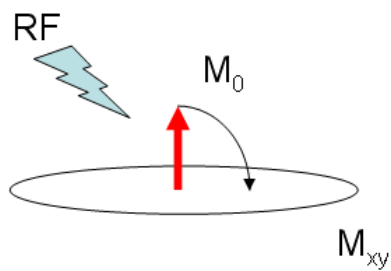

(a)

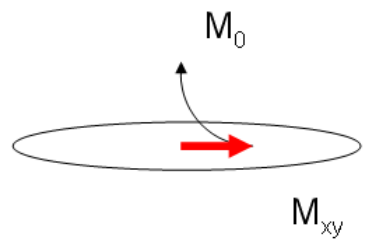

(b)

Figure 1.10. (a) Effect of the application of an RF pulse: the net magnetization $M_{0}$ is tilted and a transversal component $M_{x y}$ appears. (b) After the application of the RF pulse, the $M_{x y}$ component decreases rapidly, going back to the orginal net magnetization $M_{0}$. 
Introduction

\section{$\underline{\text { Relaxation }}$}

After $B_{1}$ is switched off, inter-nuclear and inter-molecular forces produce a loss of phase coherence among the spins. This causes a gradual loss of $M_{x y}$ (transverse relaxation) and a gradual increase of $M_{z}$ (longitudinal relaxation). These relaxation processes condition the instants at which the signal can be acquired by the receiver coil in order to obtain different contrasts among the tissues.

Longitudinal relaxation is caused by interactions between the nuclei and their environment. It is defined by a time T1, which varies according to the molecule in which the nucleus is bound and the type of tissue in which it is located. The recovery of $M_{z}$ follows and exponential rule (figure 1.11):

$$
M_{z}(t)=M_{0}+\left(M_{z}(0)-M_{0}\right) \cdot e^{-t / T 1}
$$

where $M_{z}(0)$ is the longitudinal magnetization before the application of the RF pulse. The value of T1 depends on the mobility of the molecule in which the nuclei stand. For example, in medium-sized molecules, such as lipids, the relaxation rate is faster (shorter T1) than in other molecules, such as the free water of fluids.

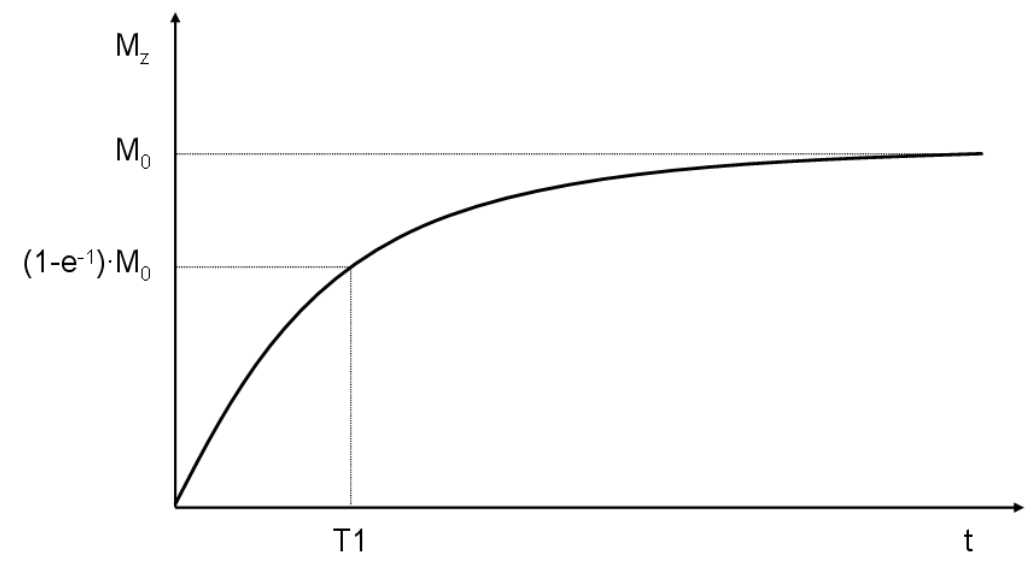

Figure 1.11. Evolution of the longitudinal magnetization after the application of an RF pulse. From this curve, the longitudinal relaxation time T1 can be calculated. 
Transverse relaxation is caused by the dephasing among the spins, originated by variations in the local magnetic field each nucleus perceives. It has two components: the microscopic environment of the nucleus (molecule size and tissue type) and the inhomogeneities of the main magnetic field. The first component takes place on a time called T2, while the second component further affects the relaxation process, shortening it to a value denoted $T 2 *$. In MR imaging, $T 2$ and $T 2 *$ can be assessed or weighted separately. Again, depending on the environment, T2 may be long (such as in free water), very short (macromolecules) or even undetectable (proteins, DNA). $2^{*}$ also depends on the presence of components with non zero magnetic susceptibility in the proximities of the scanner or the region of study (such as ferromagnetic materials, deoxyhemoglobin or hemosiderin). The $T 2^{*}$ attenuation of the transverse magnetization following an RF pulse is known as the free induction decay (FID) and is ruled by the following equation (figure 1.12):

$$
M_{x y}(t)=M_{x y}(0) \cdot e^{-t / T 2^{*}}
$$

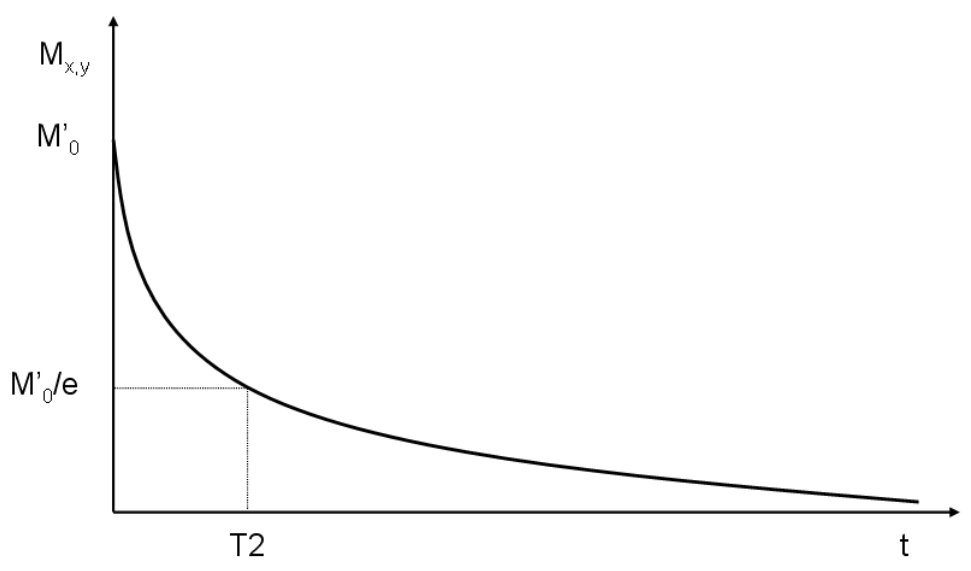

Figure 1.12. Evolution of the transversal magnetization after the application of an RF pulse. From this curve, the transversal relaxation time $T 2 *$ can be calculated.

Using an inversion pulse at $T E / 2$ it is possible to cancel the $B_{0}$ inhomogeneities and obtain a signal following $T 2$ attenuation: 
Introduction

$$
M_{x y}(t)=M_{x y}(0) \cdot e^{-t / T 2}
$$

This is called a spin-echo (SE) acquisition and it will be later described in the sequences section.

\subsubsection{Equipment}

\section{Main magnet}

The main magnet is the responsible for the creation of the static magnetic field $B_{0}$. In MR imaging it is necessary to work with extremely strong and stable magnetic field intensities (in the order of a few teslas), much higher than earth's (about 100,000 times higher). Most current clinical MR equipments use 1.5 T or 3.0 T.

Although there are several types of magnet, the most used are the superconductive electromagnets, made from dense niobium-titanium coils cooled at $-263{ }^{\circ} \mathrm{C}$. These coils carry very high electrical currents with minimal resistance. Depending on the necessities, it is possible to obtain different magnet architectures (figure 1.13).

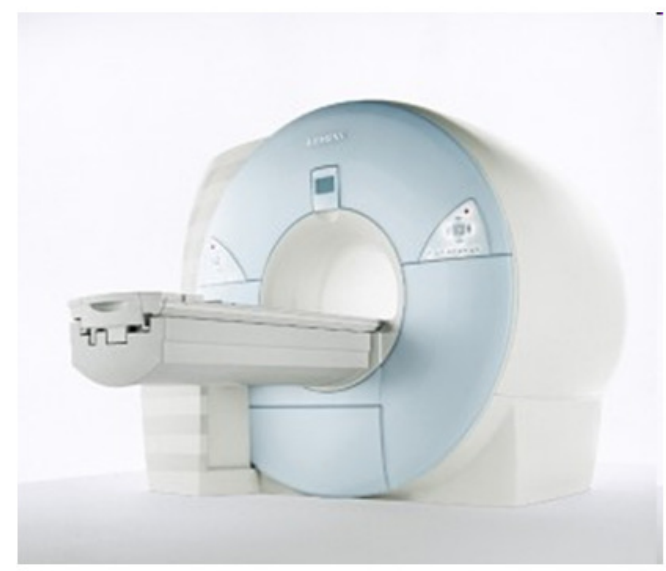

a

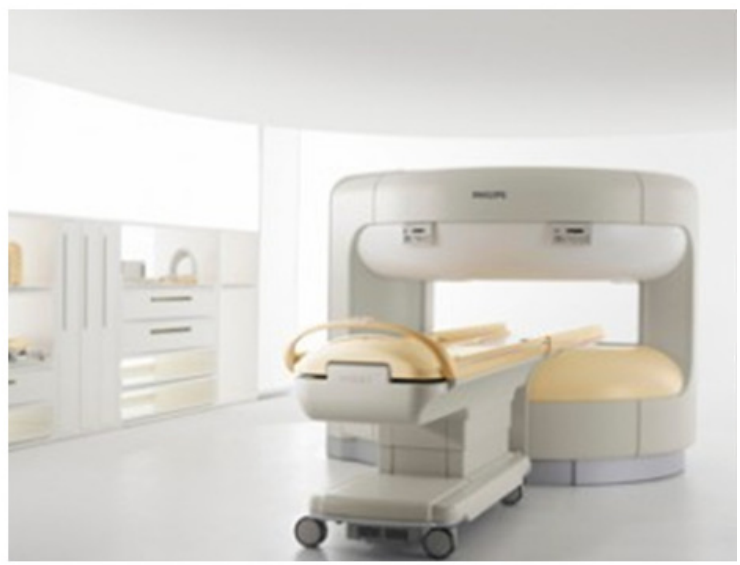

b

Figure 1.13. Clinical MR equipments (Source: a) www.medical.siemens.com and b) www.philipsmriequipment.com. Last access: January 2010). 


\section{Gradient and shim coils}

Main magnets may have small spatial variations in $B_{0}$. These effects can be corrected with gradient and shim coils, which produce compensatory magnetic fields to keep $B_{0}$ homogeneous.

Gradient fields are also used to select the parts of the subject which need to be imaged and to encode the spatial information of the signal. MR systems have three gradient coils, used to produce linear variations of the magnetic field in the three axes $X, Y$ and $Z$. These variations (in the order of the $\mathrm{mT} / \mathrm{m}$ ) allow the selection of multiple study planes. The higher the strength of a gradient the faster the acquisition and the spatial resolution of the images.

\section{$\underline{\text { RF coils }}$}

RF coils are used both to produce the excitation $\left(B_{1}\right)$ and to receive the relaxation signal. Separate coils can be used for transmission and reception, or a single coil may be used for both. Depending on the application, it is possible to use surface or volume coils. The dimensions of the coils must also be adapted to the size of the tissue under study.

\section{Computer system}

A computer system is also necessary to process all the signals and keep the system synchronized. With this system it is possible to configure the necessary pulse sequences to transmit and receive all the signals. It also reconstructs the images from the received signals and allows the configuration of planes and other acquisition parameters. 


\subsubsection{Image formation}

\section{Slice selection}

A gradient introduces a linear spatial variation in $B_{0}$ so that only a slice of the specimen under study is centered at the Larmor frequency corresponding to the RF excitation pulse (figure 1.14). The thickness of the slice is a function of the RF pulse bandwidth and the amplitude of the gradient. Varying the RF pulse central frequency it is possible to select adjacent slices. Finally, the orientation of the slice is selected by any linear combination of the three gradient coils.

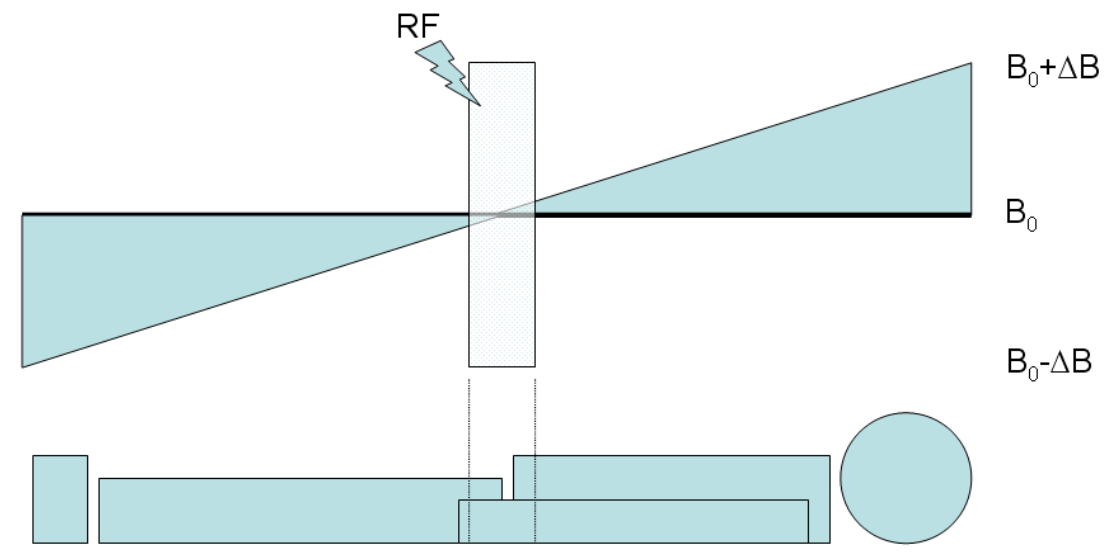

Figure 1.14. Slice selection using the combination of a magnetic field gradient and an RF pulse. It can be seen that only one section (slice) of the subject is excited, depending on the central resonant frequency and the RF pulse bandwidth.

\section{Spatial encoding}

Once a slice is selected and the RF pulse is applied, the emitted signal will be a contribution from all the nuclei of that slice. To identify the signal contribution from each voxel it is necessary to encode into the signal some spatial information to locate the voxel ( $\mathrm{x}$ and $\mathrm{y}$ coordinates). This can be done by applying two additional magnetic field gradients: the frequency encoding and the phase encoding gradients (figure 1.15). 


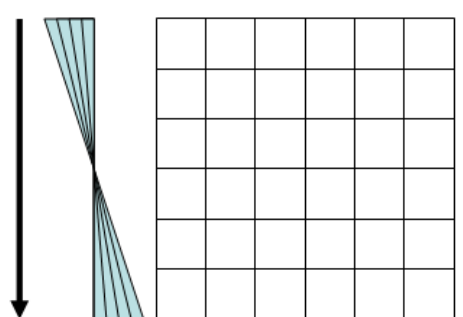

(a)

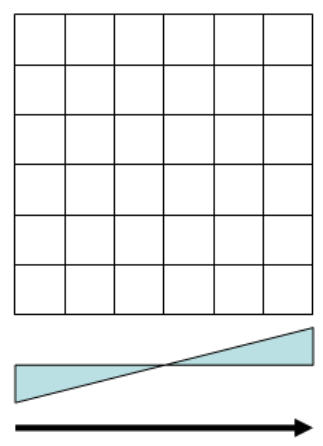

(b)

Figure 1.15. Application of (a) a phase encoding gradient and (b) a frequency encoding gradient to encode each voxel of the final image.

The phase encoding gradient is applied as a brief pulse before data acquisition. This gradient is applied during a very short time; altering the Larmor frequency of the spins and making them precess at slightly different speeds. When it is switched off all the spins go back to the same precessing frequency, but the net result is a phase variation among the spins, which is introduced in their signals. The gradient is applied several times with different amplitude to encode all the rows of the $k$-space (figure 1.16), a matrix of values that stores all the image spatial information.
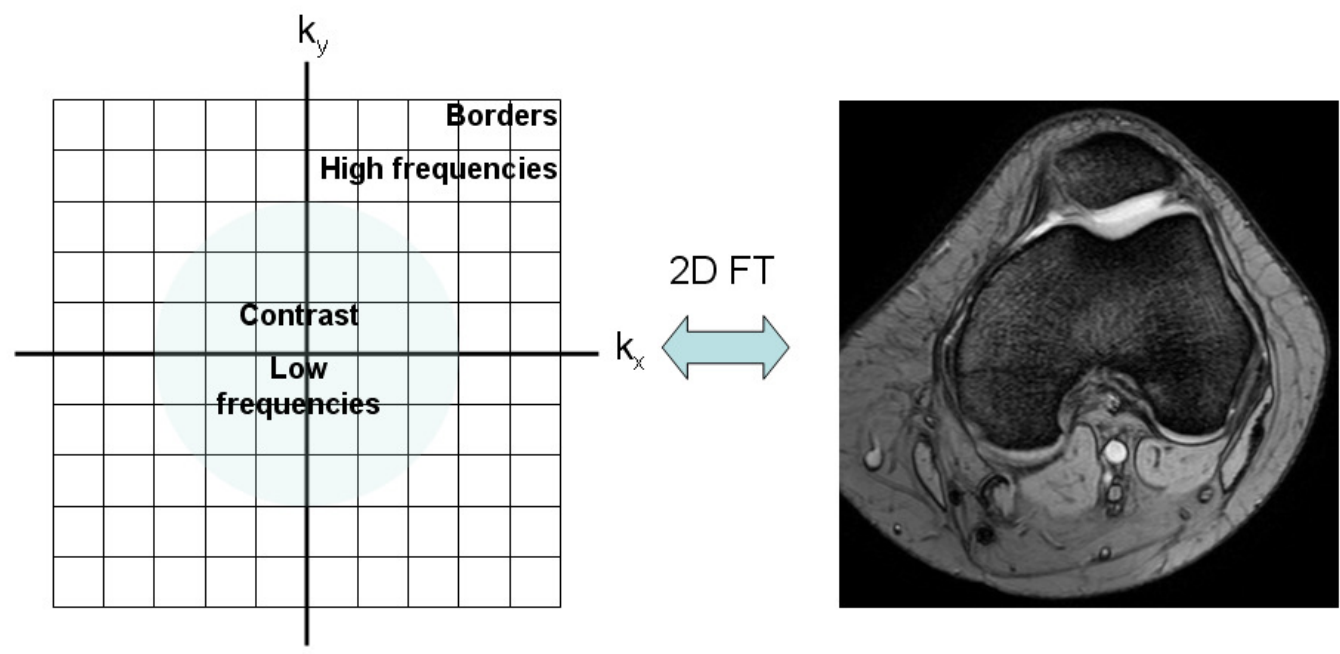

Figure 1.16. $k$-Space is related to the image with the two-dimensional Fourier transform. It can be seen that all the information of the image is previously acquired by filling the $k$-space, both with contrast (low spatial frequencies) and borders (high spatial frequencies) information. 
The frequency encoding is applied during signal acquisition. The position of the spins along the direction of the gradient can then be identified by the frequency of their emitted signals. This gradient is bipolar (positive and negative gradient) to prevent an additional dephasing of the spins.

\section{Image reconstruction}

After acquiring all the signals from the selected slice, the $k$-space is filled with values, each value corresponding to a different frequency/phase pair. By applying an inverse 2D Fourier transform to the $k$-space matrix the image is finally obtained. Information is arranged in the $k$-space so that data near the center correspond to low spatial frequencies (image contrast information) while data far from the center correspond to high spatial frequencies (image borders information).

The resolution of the resulting image is determined by the highest acquired spatial frequency, and the $x-y$ spatial resolution is usually isotropic, as the highest acquired spatial frequency is usually the same for both dimensions.

\subsubsection{Sequences}

To acquire an MR image through a Cartesian grid of $k$-space, it is usually necessary to send and acquire several RF pulses and signals in synchronization with the activation of the spatial encoding gradients. This process is called a pulse sequence. Basically, there are two big families of pulse sequences, based on the way the echo of the MR signal is formed: spin-echo (the echo is formed using rephasing $180^{\circ} \mathrm{RF}$ pulses) and gradient-echo (the echo is formed using rephasing gradients) sequences. 
Spin-echo sequences

The time diagram of a spin-echo sequence can be seen in figure 1.17:

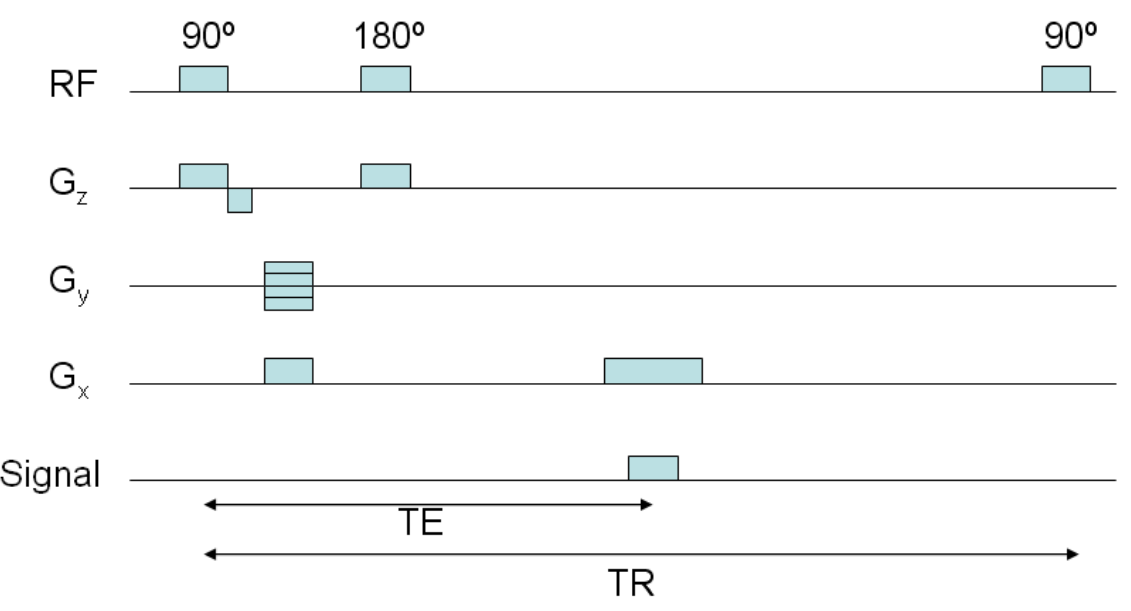

Figure 1.17. Spin echo sequence.

It can be seen that two RF pulses are used inside the same repetition time (TR). The first pulse tilts the equilibrium magnetization $90^{\circ}$ on the transverse plane, while the second pulse is an inversion pulse $\left(180^{\circ}\right)$ that refocuses spin dephasing. This second pulse is transmitted at half the time of the echo acquisition (TE/2). The slice selection gradient $\left(G_{z}\right)$ is activated each time an RF pulse is sent. It can also be seen that the echo is acquired during the activation of the frequencyencoding gradient $\left(G_{x}\right)$ and that the phase-encoding gradient $\left(G_{y}\right)$ modifies its amplitude in each $T R$ period.

In spin-echo sequences it is possible to obtain T2-weighted images, because the refocusing RF pulse cancels $B_{0}$ inhomogeneities. However, in order to acquire this type of images it is necessary to use long TRs, a fact that makes these sequences very time consuming. In order to improve speed, several echoes can be acquired in the same TR by sending additional refocusing pulses (fast spinecho sequences). 
Introduction

\section{Gradient-echo sequences}

The time diagram of a gradient-echo sequence can be seen in figure 1.18:

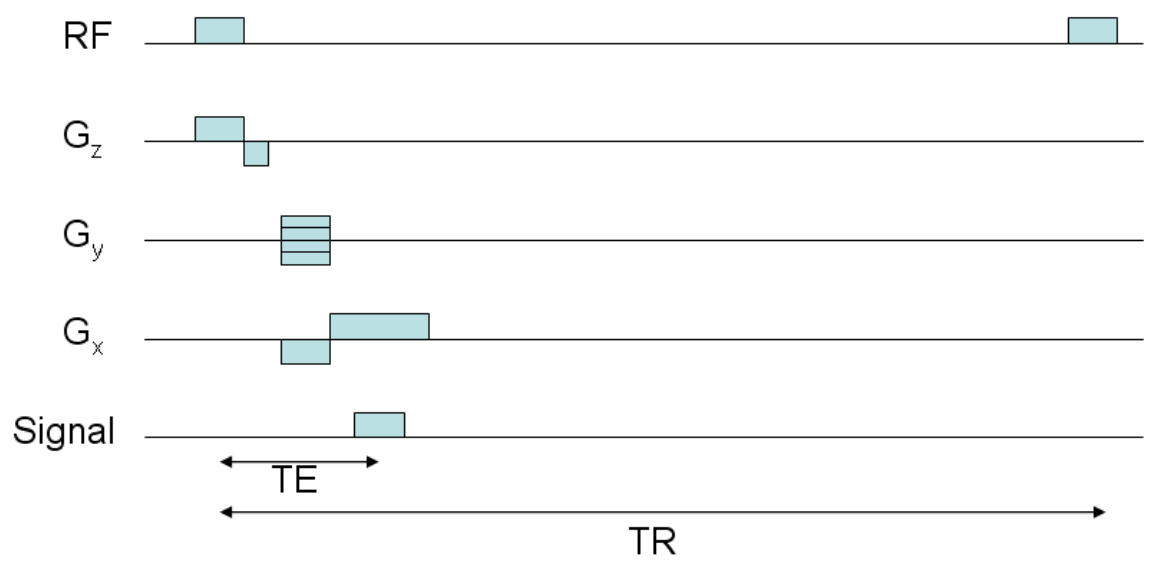

Figure 1.18. Gradient echo sequence.

In gradient echo sequences only one RF pulse is transmitted at the beginning of the TR period. In this sequence, the refocusing of the spins is produced by the frequency-encoding gradient, which minimizes it (achieves a signal maximum) at the center of the acquisition period. In fact, the frequency-encoding gradient produces a negative refocusing effect (preparatory gradient pulse) before applying the positive gradient value. This is done to achieve control of the dephasing process of the spins.

In opposition to spin-echo sequences, transverse magnetization is subject to $T 2 *$ dephasing in gradient-echo sequences. Therefore it is not possible to correct $B_{0}$ inhomogeneities. It is also common to use other flip angles (typically from $10^{\circ}$ to $40^{\circ}$ ) for the RF pulses to shorten the acquisition time.

\section{$\underline{\mathrm{PD}, \mathrm{T} 1 \text { and } \mathrm{T} 2 / \mathrm{T} 2 * \text { weighting }}$}

Depending on the combination of $T E, T R$ and flip angles, it is possible to maximize the contrast among practically all the tissues. When two tissues have very different $T 1$, a sequence with a short $T R$ (in the order of some milliseconds) 
and a short TE will enhance the difference in signal intensity between both tissues. The same happens in tissues with different $T 2$, but using now a long $T R$ (hundreds of milliseconds) and a long TE to maximize contrast. If a short TE is used in this later case, it is possible to obtain proton density (PD) weighted images (figure 1.19).

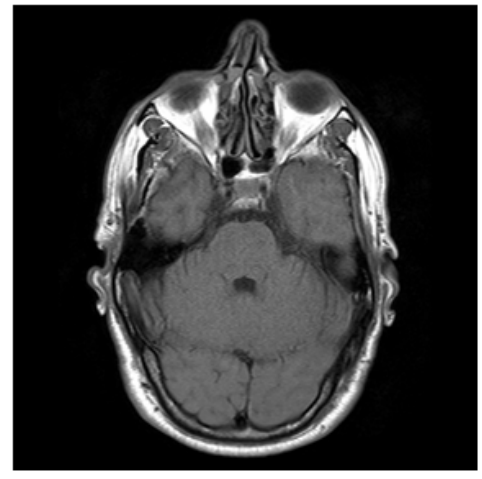

a

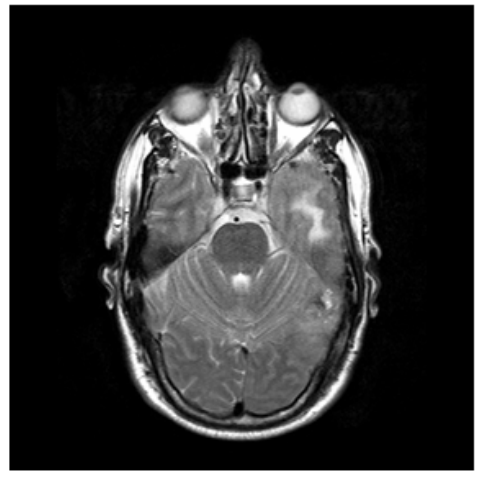

b

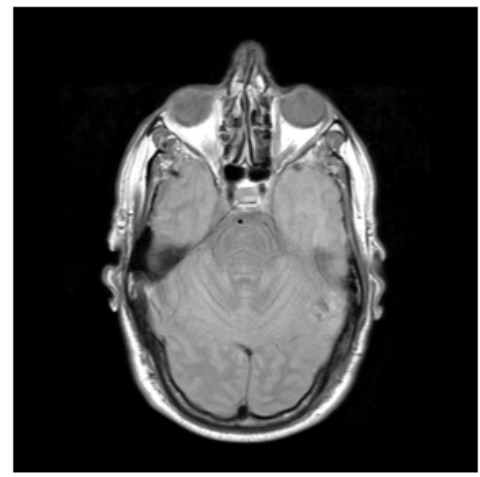

C

Figure 1.19. Examples of (a) T1, (b) T2 and (c) PD-weighted of the head.

\section{$\underline{\text { Acceleration }}$}

Modern equipments incorporate new gradient coils which allow using much faster sequences. These sequences are still based on the spin-echo and gradientecho sequences, however they take advantage of all the hardware and software potential (parallel imaging, residual magnetization suppression, $k$-space manipulation, etc.) [54,55]. Due to time and quality requirements, these are the sequences which are most commonly used in daily practice.

\subsubsection{Exogenous contrast agents}

Exogenous contrast agents alter the signal surrounding water protons via their effects on the relaxation rates. They do not contribute directly to the signal (as in $\mathrm{CT})$ but rather have an indirect action. 
They can be divided into two types: paramagnetic (based on paramagnetic ions, such as gadolinium or manganese) and superparamagnetic (based on superparamagnetic iron oxide compounds). As these ions are toxic by themselves, they need to be introduced into the body in the form of chelates, which are organic macromolecules that minimize the toxicity and characterize the effectiveness of their magnetic influence (relaxivity).

In practice, the effect of a contrast agent is the reduction of the relaxation times of the protons it finds in its surroundings. The observable effect is an increase of intensity in T1-weighted images (figure 1.20) or a decrease in T2-weighted images.
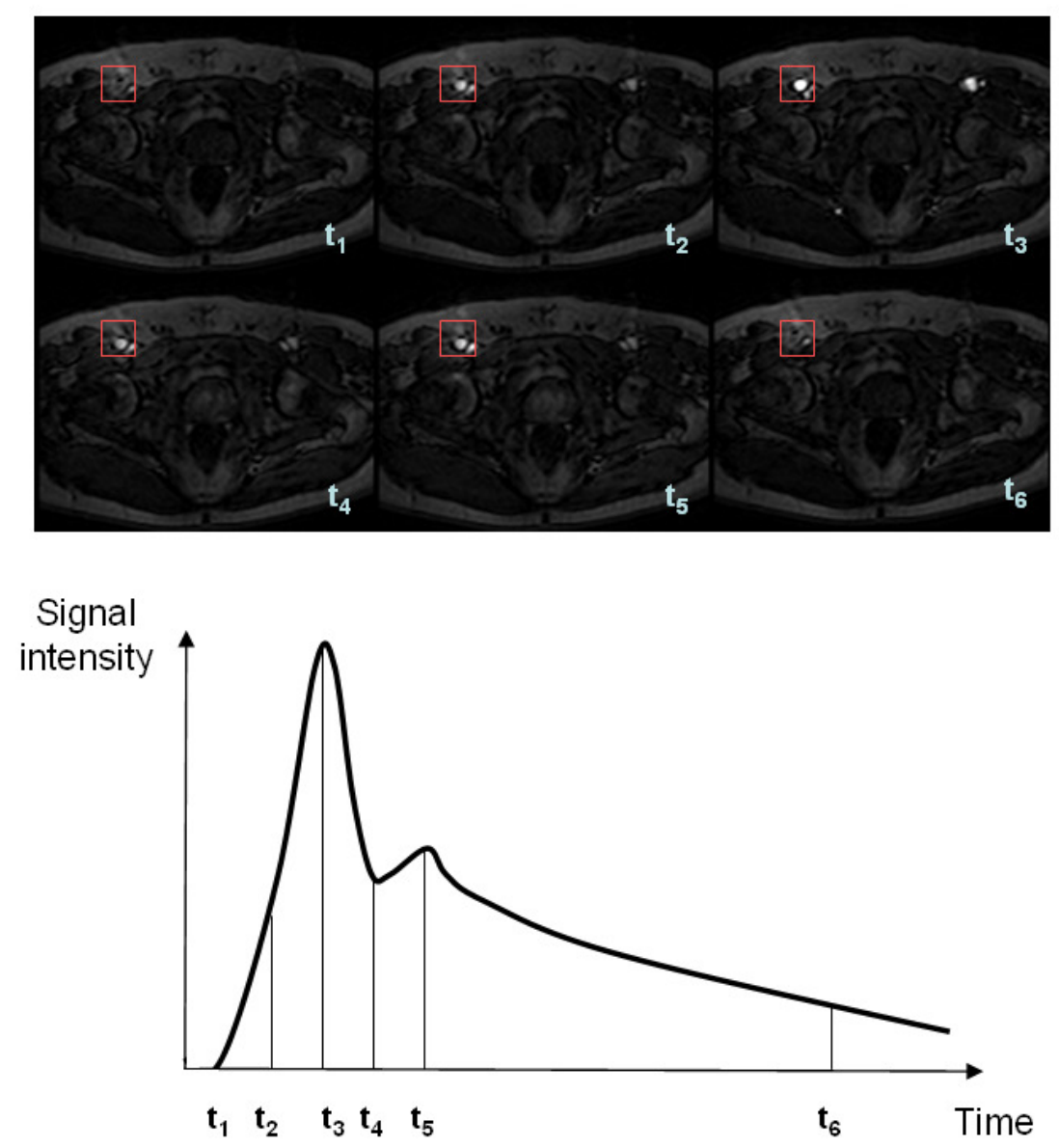

Figure 1.20. T1-weighted dynamic contrast-enhanced study of the pelvis. It can be seen that as time progresses the injected contrast diffuses from the vessels into the interstial space, producing a maximum intensity peak and then a slow washout. 
Introduction

Contrast agents are usually injected intravenously and, depending on their molecular constitution, can remain in the vascular space, diffuse into the interstitial space or penetrate cell membranes. Gadolinium-based chelates, the most commonly used contrast agents in clinical MR imaging, are extracellular. They remain in the vascular space enough time as to analyze first-pass arterial and distribution behavior and they are usually eliminated mainly through the urinary via in less than 24 hours.

\subsubsection{Acquisition requirements for a PK study}

To perform a PK study from MR perfusion images it is necessary to follow certain basic requirements. Although it is not a standardized protocol, most centers roughly coincide in the main points [5-21].

\section{Spatial requirements}

Whole region of interest coverage is desirable for a complete analysis (whole organ of interest, adjacent tissues and a major artery).

Acquired voxel size: depends on the size of the region. If possible, $\leq 1 \mathrm{~mm}$ in plane and $\leq 5 \mathrm{~mm}$ slice thickness.

\section{$\underline{\text { Temporal requirements }}$}

Sampling time: $\leq 3$ seconds, to sample the first pass of the arterial input function properly.

Total acquisition time: $\geq 5$ minutes, to sample as much as possible of the washout phase. 
Introduction

\section{$\underline{\text { Signal to noise requirements }}$}

The signal to noise ratio is clearly related to the balance between the previous requirements. Although optimized values could be obtained analytically, it is usually through trial and error that the best combination of parameters is obtained. Post-processing filters can also help to improve the results by decreasing noise.

\section{Contrast agent}

A gadolinium contrast agent (0.5 molar) is currently used, at a dose of 0.1 $\mathrm{mM} / \mathrm{kg}(0.2 \mathrm{ml} / \mathrm{kg})$ and injected at $4 \mathrm{ml} / \mathrm{s}$, followed by $40 \mathrm{ml}$ of saline flush at 4 $\mathrm{ml} / \mathrm{s}$. However this contrast dose needs to be adequate to the tissue under study, as such high concentrations may lead to saturation effects in some areas. For example, in liver studies this dose may saturate the aorta enhancement curve, so a lower dose is recommended (in our liver studies, a dose of $0.05 \mathrm{ml} / \mathrm{kg}$ is normally used).

\section{T1 mapping}

PK models work with concentration values; therefore it is necessary to calculate concentration from signal intensity. This is done by calculating the variation of T1 with the signal first, and then the variation of concentration with the T1 value. A T1 mapping sequence allows obtaining the T1 values of the tissue before the injection of contrast agent. This base value is necessary to calculate the decrease in $\mathrm{T} 1$, which is directly related to the concentration of contrast agent.

\section{Sequence}

This is an example of a 3.0 T GRE sequence used for a PK perfusion study of the knee cartilages (table 1.1): 
Introduction

\begin{tabular}{ccc}
\hline & T1 mapping & Pharmacokinetics \\
\hline Magnetic field & 3T & 3T \\
Acquisition mode & 3D sGRE & T1w 3D sGRE \\
Fat suppression & No & Yes \\
Coil & SENSE-Knee- & SENSE-Knee- 8 \\
Plane & Sagittal & Sagittal \\
Acquired voxel & $1 \times 1 \times 6 \mathrm{~mm}$ & $1 \times 1 \times 6 \mathrm{~mm}$ \\
Slices & 21 & 21 \\
Temporal resolution & - & $3,2 \mathrm{~s}$ \\
Number of & - & 64 \\
dynamics & $5 \mathrm{~min}$ & $5 \mathrm{~min}$ \\
Acquisition time & $5.4 \mathrm{~ms}$ & $3.6 \mathrm{~ms}$ \\
TE & $9.2 \mathrm{~ms}$ & $7.2 \mathrm{~ms}$ \\
TR & - & $15^{\circ}$ \\
Flip angle & $2,5,7,10,15,20,25,30,40,60^{\circ}$ & $0.2 \mathrm{ml} / \mathrm{kg} @ 4 \mathrm{ml} / \mathrm{s}$ \\
Contrast dose & - & \\
\hline Table 1.1. Typical sequences for a PK study using a $3.0 \mathrm{~T} \mathrm{MR} \mathrm{scanner.}$ \\
\hline \multicolumn{2}{c}{}
\end{tabular}

- Acquisition mode 3D sEG: gradient-echo sequence with suppression of the residual magnetization to increase speed acquisition and 3D RF excitation (the slice selection gradient selects the whole volume of study at a time).

- Fat suppression: with a combination of RF pulses and the necessary synchronization it is possible to selectively suppress the signal from fat (or any other tissue). This allows having a better contrast in the final image.

- Coil: SENSE-Knee-8. This RF coil is based on multichannel technology to speed up the acquisition process. Each channel acquires signal from a spatial sector so that the combination of all 8 channels forms the final image. Depending on the application, there are other coils with a different number of channels and architecture.

- Flip angle: the T1 mapping sequence is based on a series of images of the same volume of interest acquired maintaining the TR and the TE constant and varying the flip angle. An intensity vs. angle curve is obtain, from which the $T 1$ value can be calculated using the following equation:

$$
S(\alpha)=M \cdot \sin \alpha \frac{1-e^{-\frac{T R}{T 1}}}{1-\cos \alpha \cdot e^{-\frac{T R}{T 1}}}
$$


where $S$ is the signal intensity, $M$ is a factor containing gain and proton density information and $\alpha$ is the flip angle. T1 can be calculated by a least mean squares fit.

\subsection{Pharmacokinetic models}

\subsubsection{Definition of pharmacokinetics}

Pharmacokinetics is a branch of pharmacology dedicated to the study of the effects that the body causes on an externally administered drug. It includes the mechanisms of absorption, distribution, excretion, the time at which the drug starts to operate and the duration of its effects.

A PK analysis can be performed by two approaches:

- Non-compartmental analysis

It estimates the exposure to a drug by calculating the area under the curve of a concentration vs. time graph. It does not assume any specific compartmental model and can produce acceptable results in many studies.

\section{- Compartmental analysis}

It estimates concentration-time graphs using kinetic models. The advantage of this type of analysis is that with a model it is possible to predict the concentration of the drug at any time. However, it is often difficult to develop and validate proper kinetic models. The simplest compartmental model is the one-compartment model with intravenous bolus administration and firstorder elimination (mono-exponential tissue response). 
Introduction

1.3.2 Quantitative MR perfusion studies: from empirical measurements to PK modeling

\section{The empirical (non-compartmental) approach}

Dynamic contrast-enhanced MR (DCE-MR) studies gained popularity in the late 1980s when a series of studies gave proof that high increases and fast rates of enhancement in DCE-MR images correlated with well-vascularized tumors, in comparison to more moderate rates of normal appearing tissues or benign lesions [57-59]. These analyses focused on the morphological characteristics of the tissue enhancement curves, such as upslope, maximum, time to peak or descent (figure 1.21).

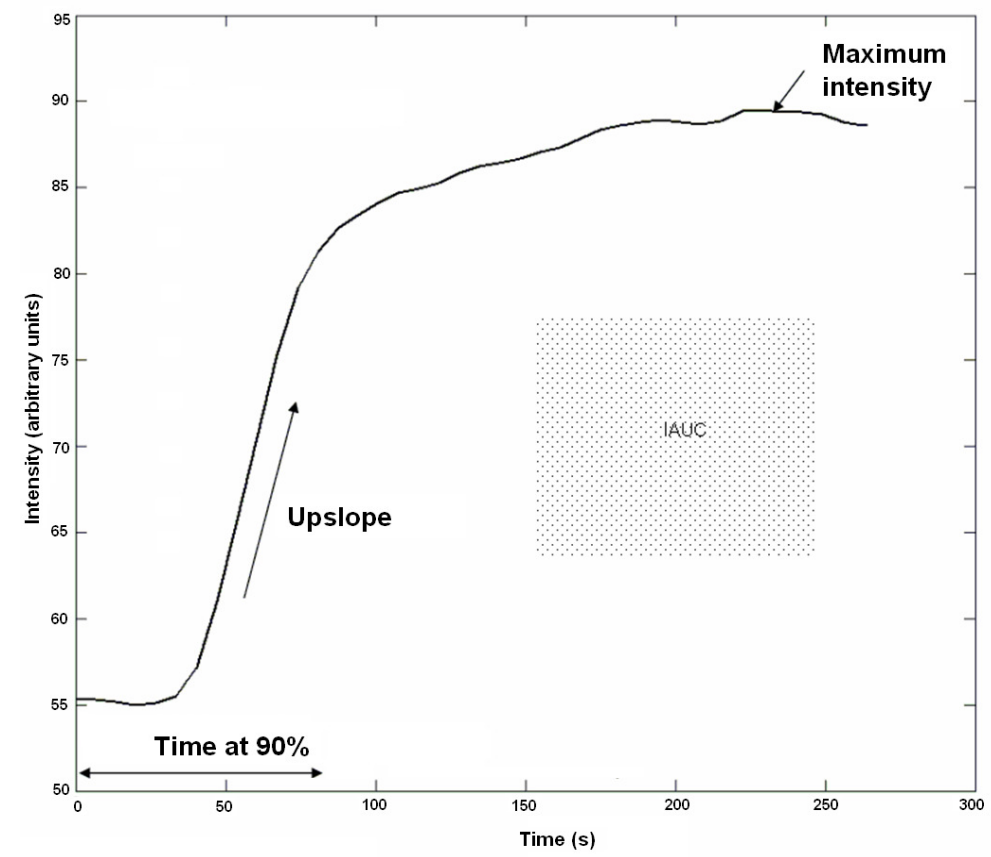

Figure 1.21. Morphologic parameters of a typical enhancement curve: upslope (speed at which the curve rises), maximum intensity, time at which the curve reaches $90 \%$ of the maximum intensity and IAUC (initial area under the curve, usually measured at $60 \mathrm{~s}$ or $90 \mathrm{~s}$ ).

These measurements are often normalized to baseline or healthy tissue signal intensity, being good lesion biomarkers [57], but they do not directly take into account physiological information. Anyway, these studies showed the necessity 
to accomplish certain temporal resolution criteria in order to sample the curves correctly. It was demonstrated that this requirement was essential for a correct extraction and characterization of the tissue enhancement curve. Temporal resolutions between 12 and $132 \mathrm{~s}$ were found to give very accurate results [60].

\section{The PK approach}

Coinciding with empirical DCE-MR studies, compartmental PK models were applied to quantify the transfer coefficients from plasma to tumor extravascular extracellular space (EES). Low molecular Gd contrasts started to be widely used during the early 1990s and they offered good properties for this type of studies.

One of the main characteristics of PK models is the use of concentration-time instead of intensity-time curves. This characteristic allows dismissing some of the disadvantages of working with intensity-time curves, such as coil and equipment gain factors. Biophysical knowledge of MR and contrast agent interactions allowed to formulate the relationship between signal intensity, relaxation rates and concentration values [61-66]. As an example, in a T1-weighted spoiled gradient echo sequence, signal intensity can be expressed as:

$$
S(\alpha)=M \cdot \sin \alpha \frac{1-e^{-\frac{T R}{T 1}}}{1-\cos \alpha \cdot e^{-\frac{T R}{T 1}}}
$$

where $M$ is a factor containing gain and proton density, $\alpha$ is the flip angle, TR is the repetition time and $T 1$ is the longitudinal relaxation time. With the variation of the signal intensity with the flip angle, it is possible to estimate T1 values with curve fitting algorithms. Then concentration can be related to T1 temporal variations caused by the magnetic influence of the contrast agent:

$$
C(t)=\frac{\frac{1}{T 1(t)}-\frac{1}{T 1(0)}}{r 1}
$$


Introduction

where $r 1$ is the relaxivity (effectiveness of the magnetic influence) of the contrast agent.

After converting intensity to concentration, the next step is to model the relationship between the concentration in the tissue and the input function, in order to study the physiological exchange of contrast agent between the vasculature and the EES. Many approaches can be taken to model this exchange, but a simple mono- or two-compartment PK model is usually adequate.

In the late 1990s a series of studies agreed on a common set of names for the PK parameters derived form the Tofts-Larsson-Brix approach [4,67]. The equations for this model are based on the conservation of mass, where $K^{\text {trans }}$ is the permeability coefficient equal to the volume transfer constant between blood plasma and EES and $k_{e p}$ is the rate of elimination or extraction from the plasma. From the so called Tofts model, the EES volume fraction can also be calculated as $v_{e}=K^{\text {trans }} / k_{e p}$, where $v_{e} \in[0,1]$ (figure 1.22$)$.

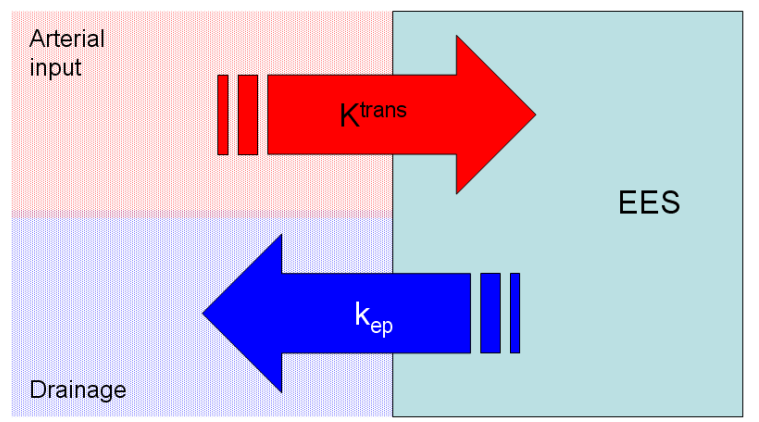

Figure 1.22. One-comparment PK model. The vascular compartment is neglected (red area) and the voxel is assumed to have enhancement only by diffusion of the contrast in the interstitial space (EES) compartment. $K^{\text {trans }}$ is the capillary permeability, $k_{e p}$ is the washout rate and EES is the extravascular extracellular space fraction.

In the review by Tofts et al. [4], three different models, assuming different conditions (high permeability, low permeability and medium permeability), were summarized: 
Introduction

High permeability or flow-limited model (Kety model [68])

This model was developed for the case of breathing an inert gas, which distributes into the whole tissue, including intracellular spaces. Two assumptions were taken: a) arteries and veins that supply and drain the tissue have well defined concentrations; and b) venous blood drains the tissue with a tracer concentration which is in equilibrium with the tissue. If the venous concentration is set equal to the EES concentration and the effect of intravascular tracer on the curve is dismissed, then the tissue concentration can be related to the plasma concentration with:

$$
\frac{d C_{t}}{d t}=F \cdot \rho \cdot(1-H c t) \cdot\left(C_{a}-C_{t} / v_{e}\right)
$$

where $C_{t}$ is the tissue concentration, $F$ is flow per unit gram of tissue, $\rho$ is the tissue density, Hct is the hematocrit (volume fraction of the whole blood occupied by cells) and $C_{a}$ is the arterial concentration.

\section{Low permeability model}

If permeability is limited and flow is high, the transport of tracer from the vasculature into the EES is slow. Therefore the rate of contrast enhancement is determined by the permeability surface area product of the capillary wall and the difference between the blood plasma concentration and the EES concentration. Again, if the intravascular contribution is dismissed, the following equation can be written:

$$
\frac{d C_{t}}{d t}=P S \cdot \rho \cdot\left(C_{a}-C_{t} / v_{e}\right)
$$

where PS is the surface area product of the capillary wall. 
Introduction

Medium permeability (mixed flow and permeability) model

When tracer uptake is limited by both flow and permeability, a new parameter can be defined: the extraction ratio $(E)$, which can be obtained as the fractional reduction in capillary blood concentration as it passes through the tissue:

$$
E=\frac{C_{a}-C_{v}}{C_{a}}
$$

where $C_{v}$ is the venous tracer concentration. This parameter can be used to characterize a tissue. Then the model equation can be written as:

$$
\frac{d C_{t}}{d t}=E \cdot F \cdot \rho \cdot(1-H c t) \cdot\left(C_{a}-C_{t} / v_{e}\right)
$$

For the previous cases, if flow is limited $(P S>>F)$ then $E=1$, and if permeability is limited $(P S<<F)$ then $E=P S /(F(1-H c t))$.

Finally, a generalized kinetic model, containing the three previous assumptions can be written as:

$$
\frac{d C_{t}}{d t}=K^{\text {trans }} \cdot\left(C_{a}-C_{t} / v_{e}\right)=K^{\text {trans }} \cdot C_{a}-k_{e p} \cdot C_{t}
$$

Or in its integration form:

$$
C_{t}(t)=K^{\text {trans }} \cdot \int_{0}^{t} C_{a}(\tau) e^{-k_{e p}(t-\tau)} d \tau
$$

where the tissue response to an ideal arterial input delta is 


$$
\text { Introduction }
$$

$$
h(t)=K^{\text {trans }} \cdot e^{-k_{e p} t}
$$

Additionally, a temporal delay $\tau$ can also be included in order to correct for onset time. However, this value can be easily corrected before the application of the PK model, without the need of including it as another fitting parameter. As it can be seen, $K^{\text {trans }}$ groups the previous concepts of permeability and flow. This decision is taken to simplify the model calculations. Additional information regarding the tissue physiology needs to be obtained qualitatively in order to interpret the quantitative values of $K^{\text {trans }}$ properly.

Other approaches also follow:

\section{Vascular contribution}

Although the above mentioned models are referenced as twocompartment models because they take into account both plasma and EES, the equations do not take into account a possible vascular contribution of the input function to the tissue enhancement curve. Therefore, an extension of the generalized kinetic model can be done, adding an extra term to the formulation containing the direct vascular contribution of the arterial input function to the tissue concentration curve (figure 1.23). This extension was developed by St. Lawrence and Lee [69] and was called adiabatic approximation:

$$
C_{t}(t)=v_{p} C_{a}(t)+K^{t r a n s} \cdot \int_{0}^{t} C_{a}(\tau) e^{-k_{e p}(t-\tau)} d \tau
$$

where $v_{p}$ is the vascular space volume fraction. 


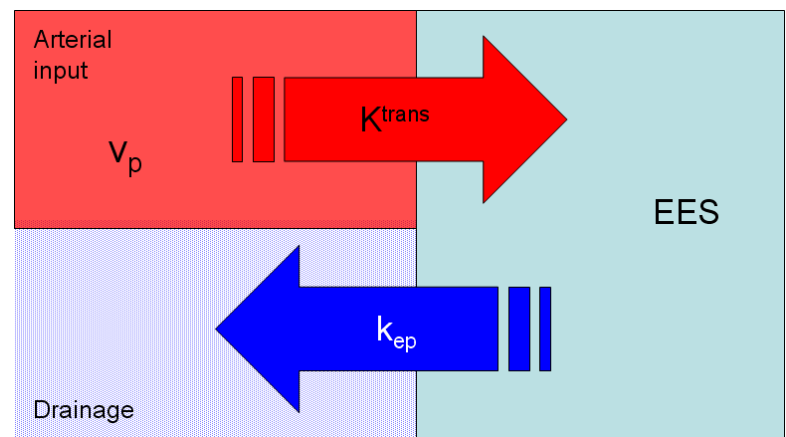

Figure 1.23. Two-comparment PK model. The vascular contribution is not neglected and the voxel is assumed to have enhancement both from the presence of vessels and by diffusion of the contrast in the interstitial space. $v_{p}$ is the vascular space fraction, EES is the extravascular extracellular space fraction, $K^{\text {trans }}$ is the permeability and $k_{e p}$ is the washout rate.

\section{First-pass or leakage profile}

Another approach was obtained to allow simultaneous mapping of endothelial permeability and blood volume in intracranial lesions [70]. It was applied to improve the quantification of blood volume in brain tumors, due to the fact that when the blood brain barrier is broken there is extravasation of contrast from the vessels into the interstitial space (leakage), so that the vascular volume may be overestimated. This effect is mostly produced in the angiogenic rim of malignant tumors. So, this approach tries to separate contributions to the signal enhancement both by the local blood volume and the capillary permeability, producing cerebral blood volume $(C B V)$ maps free from leakage effects and permeability maps which are not so affected by large blood volumes.

If in the generalized kinetic model formulation:

$$
\frac{d C_{t}}{d t}=K^{\text {trans }} \cdot\left(C_{a}-C_{t} / v_{e}\right)=K^{\text {trans }} \cdot C_{a}-k_{e p} \cdot C_{t}
$$

we assume that $C a>>C t$, as we focus only on the first pass of the contrast bolus, then, this can be written as: 


$$
\frac{d C_{t}}{d t}=K^{f p} \cdot C_{a}
$$

where $K^{\text {trans }}$ has been renamed to $K f p$ to account for first pass meaning. And its integral form:

$$
C_{t}(t)=K^{f p} \cdot \int_{0}^{t} C_{a}(\tau) d \tau
$$

which is called the leakage profile of the tumor obtained during the first pass of the contrast bolus.

Then the $C B V$ values can be obtained free of leakage effects by applying the following relationship:

$$
C B V_{\text {corrected }}=\int_{0}^{T}\left(C_{t}(t)-K^{f p} \int_{0}^{t} C_{a}(\tau) d \tau\right) d t
$$

where $T$ is the curve acquisition time. It can be noticed that $C B V$ is usually obtained as the integral of $C_{t}(t)$.

\section{The Patlak plot technique}

The Patlak plot technique describes a two-compartment model with unilateral tracer flow from compartment 1 into compartment 2 [71]. The following assumptions are made:

- Signal change is proportional to the concentration of contrast agent in a particular voxel.

- Contrast agent is promptly and completely mixed inside the compartments.

- The Hct is constant in all vessels. 
- The contrast agent concentration in the input vessel and the tissue vessels is equal at any time.

The total amount of tracer in a tissue can be expressed as the sum of contrast agent in the vascular space and the interstitial space:

$$
K(t)=B(t)+Q(t)
$$

It is assumed that the amount of contrast agent in the vascular space $B(t)$ is proportional to the concentration of the vascular input function:

$$
B(t)=c_{1} \cdot C_{a}(t)
$$

where the variable $c_{1}$ represents a constant equivalent to the vascular space. It is further assumed that the amount of contrast agent in the tissue is proportional to the integral of the concentration curve of the input function, which is:

$$
Q\left(t_{1}\right)=c_{2} \cdot \int_{0}^{t_{1}} C_{a}(t) d t
$$

where $t_{1}$ is the curve acquisition time. Therefore, $c_{2}$ is equivalent to the transfer ratio from the vascular space into the tissue. And finally,

$$
K\left(t_{1}\right)=c_{1} \cdot C_{a}\left(t_{1}\right)+c_{2} \cdot \int_{0}^{t_{1}} C_{a}(t) d t
$$

If we divide the previous expression by $C_{a}\left(t_{1}\right)$, the resulting term is the Patlak plot equation: 


$$
\begin{gathered}
\frac{K\left(t_{1}\right)}{C_{a}\left(t_{1}\right)}=c_{1}+c_{2} \cdot \frac{\int_{0}^{t_{1}} C_{a}(t) d t}{C_{a}\left(t_{1}\right)} \\
Y=c_{1}+c_{2} \cdot X
\end{gathered}
$$

Now it is possible to draw a plot using the terms $X$ and $Y$. If all assumptions are true, the plot will behave like a straight line, so that $c_{2}$ is equivalent to capillary permeability and $c_{1}$ is equivalent to the vascular space fraction.

Assumption of a biexponential decay AIF

If the AIF is assumed to have a biexponential decay [72], then it can be written as:

$$
C_{p}(t)=D \cdot \sum_{i=1}^{2} a_{i}^{T} e^{-m_{i} t}
$$

where $D$ is the dose (mmole $/ \mathrm{kg}$ ), and amplitudes $a^{T}$ are normalized for unit dose (so that $C_{p}$ is then known for any size dose). The fitted values obtained by Weinmann et al. [72] were set at $a_{1}{ }^{T}=3.99 \mathrm{~kg} /$ liter, $a_{2}{ }^{T}=4.78$ $\mathrm{kg} /$ liter, $m_{1}=0.144 \mathrm{~min}^{-1}, m_{2}=0.0111 \mathrm{~min}^{-1}$.

With this assumption, the generalized kinetic model can be written as:

$$
C_{t}(t)=D \cdot K^{\text {trans }} \cdot \sum_{i=1}^{2} a_{i}^{T} \frac{e^{-k_{e p p} t}-e^{-m_{i} t}}{m_{i}-k_{e p}}+v_{p} \cdot D \cdot \sum_{i=1}^{2} a_{i}^{T} e^{-m_{i} t}
$$


Direct use of signal intensity curves (Brix model)

According to this approach, the measured signal-intensity curves are analyzed within the framework of PK modeling [63,73]. It can be mathematized by the following equation:

$$
\frac{S(t)}{S(0)}=1+A \cdot\left\{\left(e^{k_{e p} t^{\prime}}-1\right) \cdot e^{-k_{e p} t}-\left(e^{K^{\text {trans }} t^{\prime}}-1\right) \cdot e^{-K^{\text {trans }} t}\right\}
$$

where $A$ is an amplitude correcting factor and $t^{\prime}$ is the time for bolus infusion of contrast agent.

\section{Dual input model}

Most cases can be analyzed with the previous equations, using one-input two-compartment models. However, in the special case of the liver, a dual blood supply has to be taken into account: hepatic artery and portal vein. To include this dual input, the generalized kinetic model can be written as [74]:

$$
C_{t}(t)=\int_{0}^{t}\left(K^{\text {trans } 1} C_{a}(\tau)+K^{\text {trans } 2} C_{v}(\tau)\right) e^{-k_{e p}(t-\tau)} d \tau
$$

or, if we include the vascular component:

$$
C_{t}(t)=v_{p 1} C_{a}(t)+v_{p 2} C_{v}(t)+\int_{0}^{t}\left(K^{\text {trans } 1} C_{a}(\tau)+K^{\text {trans } 2} C_{v}(\tau)\right) e^{-k_{e p}(t-\tau)} d \tau
$$

where terms 1 and 2 refer to separate blood contributions (hepatic artery and portal vein, respectively). 
The generalized kinetic model has been widely used in a number of studies, covering several regions and tumor types, such as breast [18], liver [74], kidneys [75], musculoskeletal system [76], prostate [77], brain [78] and ovaries [79]. In this context, it has proved to be a useful tool for the assessment of disease and treatment efficacy [2]. However, different model assumptions, MR acquisition protocols and post-processing algorithms introduce an important amount of variability sources which need to be addressed carefully:

\section{Input function selection}

In many studies an assumed input function is used, based on the work by Weinmann [72], where a biexponential decay curve reflecting the change in plasma concentration with time due to exchange and renal excretion was published (figure 1.24). However, this curve does not provide firstpass information (bolus pass).

The use of tissue-specific vascular input functions, individually measured near the tissue of interest (either manually or through automatic algorithms), provides further information about the first-pass phase, taking into account both the cardiac output and the hemodynamics of the patient (figure 1.24). This provides more realistic information about the tissue physiology. Further steps may require curve fitting to a gamma function [80] or ICA-extracted (independent component analysis) curves [81].

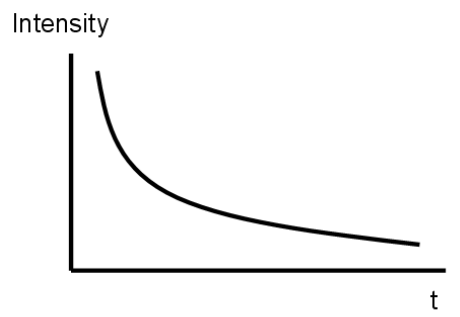

(a)

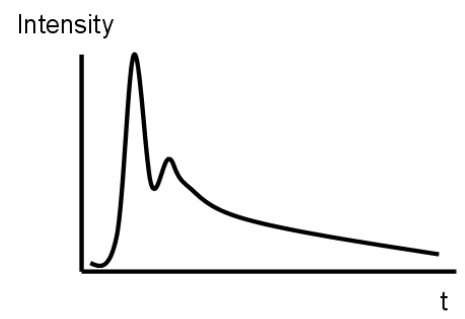

(b)

Figure 1.24. Examples of different arterial input functions: (a) standardized experimentally derived biexponential decay function and (b) individually derived arterial input function. 
Ideally, the vascular input function to the PK model should be that of the capillary branch which irrigates a certain voxel (on a voxel-based approach). However, at the moment this is technically impossible, due to spatial resolution limitations. Therefore a consensus needs to be found in order to obtain reproducible results. A good approach is to take as input function the enhancement curve of the nearest large vessel from which the tissue is irrigated. As examples, the hepatic artery for the liver, the popliteal artery for the patellar cartilage or the iliac artery for the prostate.

\section{Vascular contribution}

As explained above, the traditional PK models (Tofts, Larsson, Brix) do not take into account a vascular contribution in the formulation. This may be appropriate in most tissues, where the arterial component of the enhancement curve can be easily neglected. However, in tumors, especially those with high vascularization, the arterial contribution is very significant. To model this contribution, a different model should be used (i.e., the St. Lawrence and Lee adiabatic approximation). The choice of one model or another requires previous knowledge about the physiological response of the tissue.

\section{Intensity to concentration conversion}

Although the intensity to concentration conversion is independent of the PK model, it is also necessary to find a consensus approach. Most recent studies use multiple flip angle sequences to estimate pre-contrast T1 and its variation during contrast injection [82]. From this variation the concentration can be easily calculated with the linear relationship shown above.

Other approaches use a phantom-derived polynomial conversion [7] or a linear conversion [17]. 


\section{Image acquisition protocol}

Spatial and temporal requirements, as stated in previous points, are also an important source of variability, as modern equipments may allow deeper insights which some centers may not be able to reach yet. Low sampling rates may lead to undersampling the first pass of the vascular input function, therefore overestimating the value of the PK parameters. Also, low spatial resolution may lead to partial volume effects, making it impossible to detect small lesions and underestimating heterogeneity.

Other factors, such as the acquisition duration, the contrast agent dose and injection rates, the magnetic field strength and patient's collaboration (especially in abdomen studies to decrease movement contribution) are further sources of variability which need consensus.

\section{Image analysis}

The use of average enhancement curves from the whole tissue or curves extracted from each voxel offers different results when it comes to characterize a tissue or a lesion. Average curves are more noise-proof but they also introduce average artifacts, losing the capacity to analyze tissue heterogeneity as they minimize outliers (which may be of special interest for the study of tumors). On the other hand, a voxel-based analysis allows studying tissue heterogeneities with parametric colored maps, but with noisier curves (an optimal balance between spatial and temporal resolutions is critical here). In this later approach, the use of image registration and spatial and temporal filters may help to improve the quality of the curves. It is important to notice that better curves (i.e., smoother curves with less noise or oscillations) will provide better results when fitting the curves and extracting the PK parameters. 


\section{Curve fitting}

As PK parameters do not have an analytical expression, it is necessary to use curve fitting strategies to estimate them from the vascular and tissue concentration curves. Most studies use non-linear least squares fitting algorithms, mainly the one developed by Levenberg-Marquardt [83]. However, curve fitting algorithms are usually dependent on initialization values, which may lead to local minima and wrong results. Other fitting techniques allow specifying lower and upper bounds for the parameters, which may bring huge amounts of saturated values. The study of the residuals and the incorporation of complementary clinical knowledge are essential to state the quality of the results and dismiss possible fitting mistakes.

\section{Statistical analysis}

The results of an analysis are usually given as the mean (or the median) and the standard deviation of the region under study. This is appropriate in those cases where the region is relatively homogenous or when the only interest is to obtain a gross estimation. However, histogram analysis may provide richer information when studying highly heterogeneous regions, such as tumors. Handling a histogram allows to group the distribution of values into percentiles as well as estimating higher order statistics like the skewness and the kurtosis, which are directly related to the histogram shape and, as a consequence, to the region heterogeneity.

Another characteristic of the studies is the use of bivariate analysis (such as Student's t-test or ANOVA), where each PK parameter is analyzed separately for statistical significance. The use of multivariate analysis may provide higher statistical power in order to characterize tissues. 


\section{Methodology}

\subsection{Image preparation}

\subsubsection{Image filters}

MR perfusion studies are four-dimensional acquisitions where a volume of interest is acquired several times during the study. This characteristic allows using two types of filters: spatial and temporal. A spatial filter tries to reduce the noise of an image at a certain instant, either in $2 \mathrm{D}$ or $3 \mathrm{D}$, depending on the implementation. A temporal filter tries to reduce the noise of the enhancement curves of each voxel, as it is expected that ideal curves will follow smooth enhancement patterns without oscillations. In both types of filters the main aim is to increase the signal to noise ratio without losing essential information (such as an arterial peak or a small lesion).

\section{$\underline{\text { Noise in MR images }}$}

When working with MR images, it is necessary to know the probability density functions (PDF) of the data, which describes the relative likelihood of a random variable to occur at a given point in the observation space. Wrong assumptions about how the data is distributed may lead to erroneous results when the postprocessing algorithms are applied.

In general, a Gaussian PDF is defined by (figure 2.1):

$$
P(x \mid \mu, \sigma)=\frac{1}{\sqrt{2 \pi \sigma^{2}}} e^{-\frac{(x-\mu)^{2}}{2 \sigma^{2}}}
$$


where $\mu$ is the mean and $\sigma$ is the standard deviation of the PDF. However, the MR data acquired in the $k$-space are complex and they are formed of noiseless signal plus white noise contributions (additive, independent and characterized by a zero-mean Gaussian PDF). Then, after applying the inverse Fourier transform, the complex data resulting from the transformation is still Gaussian, due to the linearity and orthogonality of the FT. Its PDF can be expressed as:

$$
P\left(\omega_{r}, \omega_{i} \mid A, \varphi, \sigma\right)=\frac{1}{\sqrt{2 \pi \sigma^{2}}} e^{-\left(\frac{\left(\sigma_{r}-A \cos \varphi\right)^{2}}{2 \sigma^{2}}+\frac{\left(\sigma_{i}-A \sin \varphi\right)^{2}}{2 \sigma^{2}}\right)}
$$

where $\omega_{r}$ and $\omega_{i}$ are the real and imaginary variables, respectively, $A$ is the amplitude and $\varphi$ is the phase.

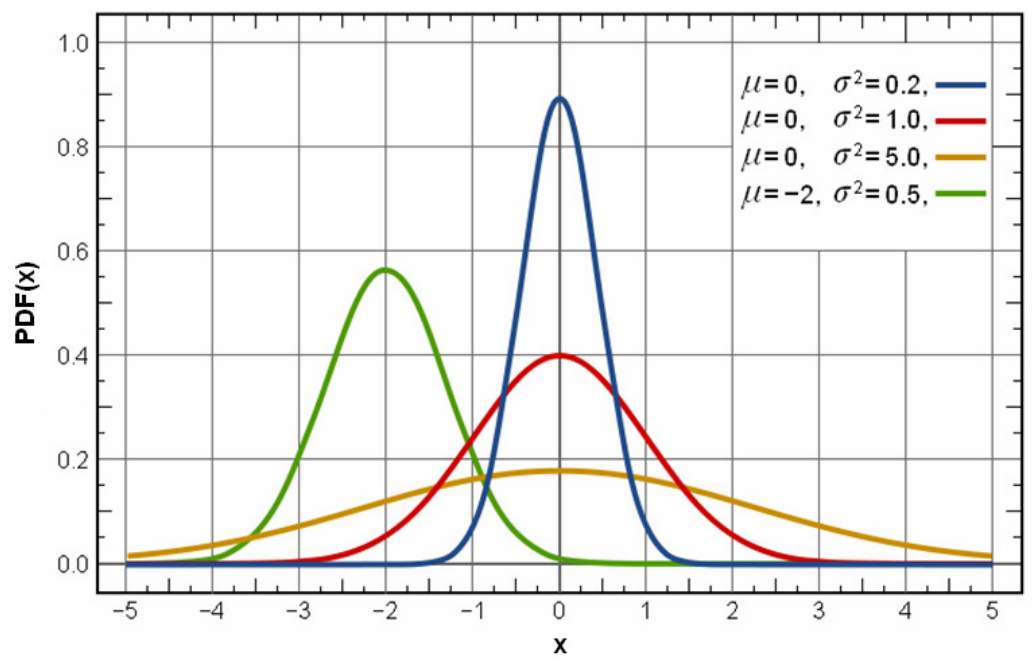

Figure 2.1. Probability density functions of a Gaussian distribution

When MR images are processed it is common to work with magnitude data instead of real and imaginary data, because magnitude data have the advantage of being immune to incidental phase variations caused by $B_{1}$ shimming variations. The magnitude of a complex number can be calculated as: 


$$
m=\sqrt{\omega_{r}^{2}+\omega_{i}^{2}}
$$

As it can be seen, this is not a linear operation, so the result is not expected to be Gaussian distributed anymore. The PDF of the magnitude data can be obtained from the Gaussian PDF by transforming real and imaginary data into polar coordinates, giving the Rician PDF (figure 2.2):

$$
P(x \mid A, \sigma)=\frac{1}{\sigma^{2}} e^{-\frac{x^{2}+A^{2}}{2 \sigma^{2}}} I_{0}\left(\frac{A}{\sigma^{2}}\right) \mathcal{E}(x)
$$

where $I_{0}$ is the zeroth-order modified Bessel function of the first kind, $\varepsilon$ is the unit step Heaviside function and $x$ has to be positive. The shape of the Rician PDF depends on the ratio $A / \sigma^{2}$, which corresponds to the signal-to-noise ratio (SNR). When the SNR is high, the Rician PDF is similar to a Gaussian PDF.

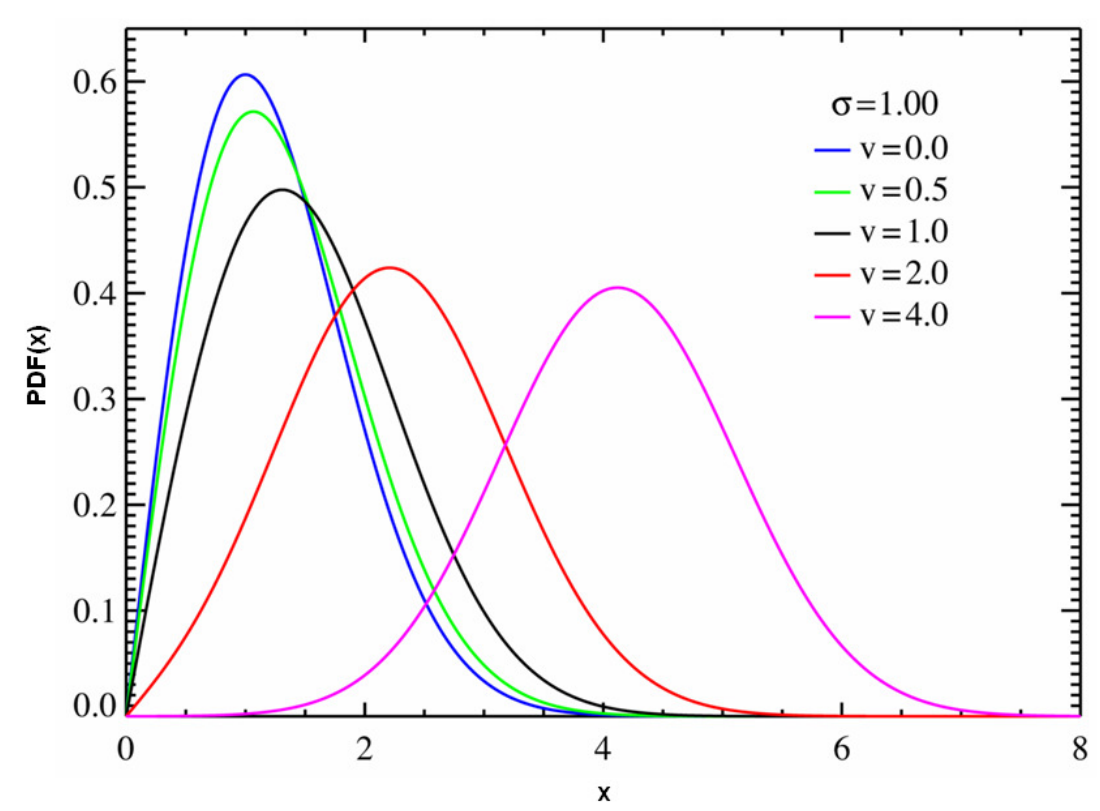

Figure 2.2. Probability density functions of a Rician distribution 
To estimate signal amplitude and noise variance in MR images several approaches can be taken [85]: mean squared error, Cramér-Rao lower bound or maximum-likelihood. However, it is not always assumed that the magnitude images have Rician PDFs, so that in some cases the estimations may be mistaken. As to where the signal and noise should be estimated, these are some recommendations [85]:

- The signal amplitude should be estimated from complex data, if the true phase values are constant; or from signal amplitude data, if the true phase values are unknown.

- The image noise should be estimated from the background (where magnitude is zero), so it can be estimated either from complex or magnitude data.

As it can be seen, accurate estimations of $S N R$ s are essential to assess the quality of MR images, in order to maintain a proper balance between $S N R, C N R$ (contrast-to-noise ratio) and resolution.

\section{Spatial filters}

Two features are desirable from a spatial filter for MR images: to significantly decrease image noise and to preserve fine details.

In MR images, it is essential to use filters which are capable of distinguishing anatomical regions throughout the image. In the literature several types of filters are available: adaptive filters [85], wavelet filters [86], non-local means [87] or anisotropic diffusion filters [88]. Spatial filtering is usually applied with two assumptions: the image is formed of several regions where the signal is stationary and ergodic in mean and variance; and the image noise is Gaussian distributed and with zero mean. However, as stated in the previous section, MR images have Rician distributions and this can lead to erroneous results when images have low SNR. Some of the above filters can be also used in this context (wavelet filters) or can be adapted (anisotropic diffusion [89]). 


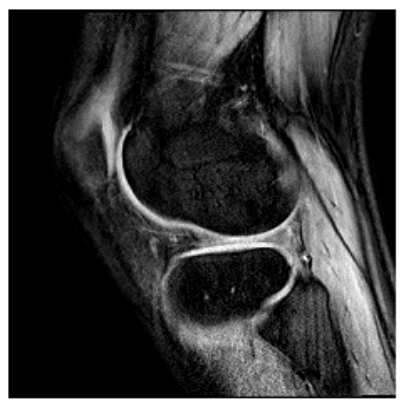

Original image

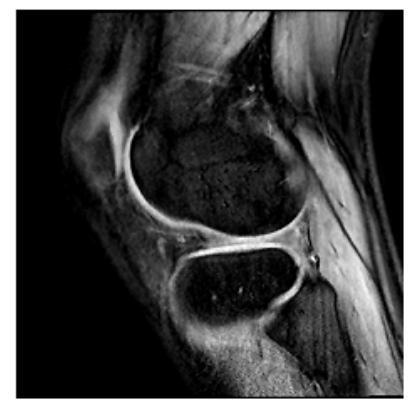

Adaptive filter (Wiener 3×3)

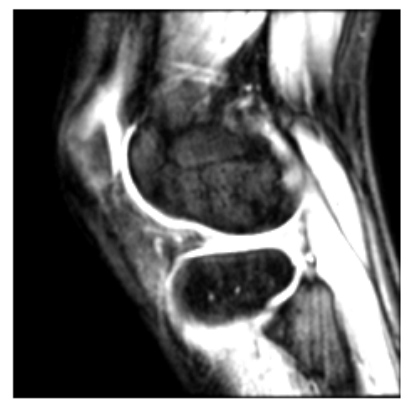

Anisotropic filter

(iters $=5$, delta $=1 / 7$, kappa $=30$ )

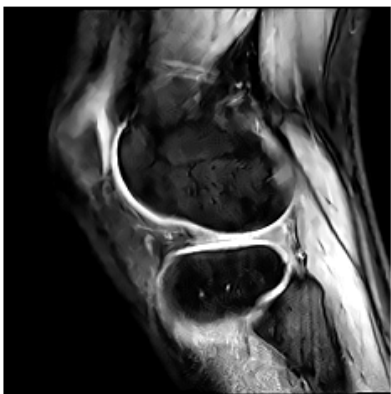

Non-local means
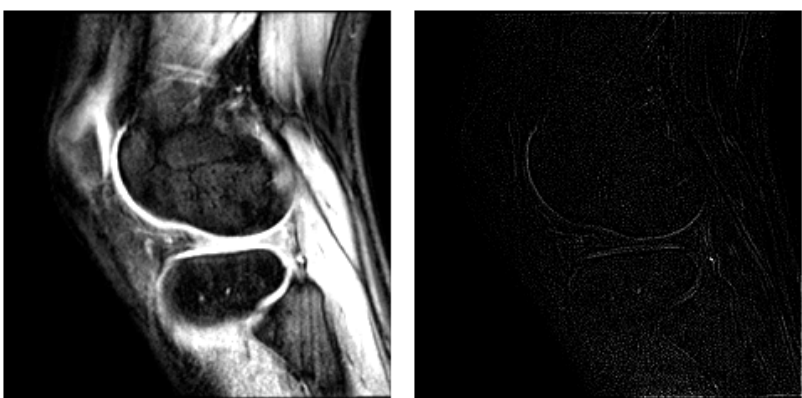

Gaussian filter

$(5 \times 5$, sigma $=1)$

Figure 2.3. Examples of image filters (central column) with their residuals (right column). It can be seen that most residuals still contain information of the original image, especially contours. 
Before applying spatial filters it is also necessary to know how the noise is distributed. MR images are often acquired with parallel techniques and they are also conditioned by RF variations. This causes signal intensity inhomogeneities which need to be corrected with special filters [90,91].

It is important to notice that applying optimal image filtering methods may be essential for accurate image segmentation. The scope of this thesis is not based on image segmentation techniques but on the functional evaluation of tissue behavior through time. However, for coherence, some of the above filters were tested with DCE-MR images to evaluate their performance (figure 2.3). The best results were obtained for the non-local means approach, although its current implementation was very time consuming. For speed and simplicity, a fast Gaussian smoothing filter (with $n=5, \sigma=1$ ) was applied in all the clinical applications.

\section{Temporal filters}

The PK analysis of DCE-MR images is based upon the study of time enhancement curves. As it depends on the application of curve fitting methods, these curves should be as noise-free as possible.

The enhancement curve of a voxel is obtained as the signal intensity value of that voxel at each dynamic image with spatial coherence. Depending on the tissue under study, a curve will have one level of noise or another, understanding noise as arbitrary oscillations of the curve. Several smoothing methods can be applied to reduce this noise:

\section{Moving averages}

Moving averages can be defined as a finite impulse response filter that creates a series of averages from different subsets of the original series of values. Therefore, each value of a moving averages filtered curve is the result of averaging a subset of values (usually a few ones) from the 
extracted curve. As input parameter, it is necessary to specify the window size (number of values to be averaged). The higher the window (span) the smoother the resulting curves.

This filter reduces oscillations effectively but it has undesired effects when applied to arterialized enhancement curves, where there is a signal peak in the curve which lasts for one or two dynamics. The height of this peak will be drastically reduced as it is averaged with the rest of values, therefore losing essential information for an accurate PK analysis.

\section{Local regression (LOESS and LOWESS or weighted LOESS)}

Local regression, also known as locally weighted polynomial regression, uses low-degree polynomials (usually grades 1 for a linear approximation or grade 2 for a quadratic approximation) to fit subsets of the data [92]. The polynomial is fitted using weighted least squares, giving more weight to values near the point which is to be fitted and less weight to more distant values. The value of the filtered curve for a certain point is then obtained by evaluating the local polynomial. As input parameters, it is necessary to specify the degree of the polynomial and the weights. Higher degree polynomials can theoretically be used, but in practice they would tend to overfit the data in the local environment.

In comparison to the moving averages, LOESS, specifically 2nd degree polynomial LOESS, offers better results when dealing with arterialized enhancement curves, as the arterial peak is much more respected.

\section{Savitzky-Golay filter}

The Savitzky-Golay filter is a generalized moving average filter where the filter coefficients are obtained by performing an unweighted linear least squares fit using a polynomial of a specified degree [93]. 
Again, using the Savitzky-Golay filter the arterial peaks of the curves can also be preserved, in comparison to the moving averages.

Finally, after comparing the performance of these smoothing filters, the best results were drawn from the LOESS filter with 2nd degree polynomials (figure 2.4).

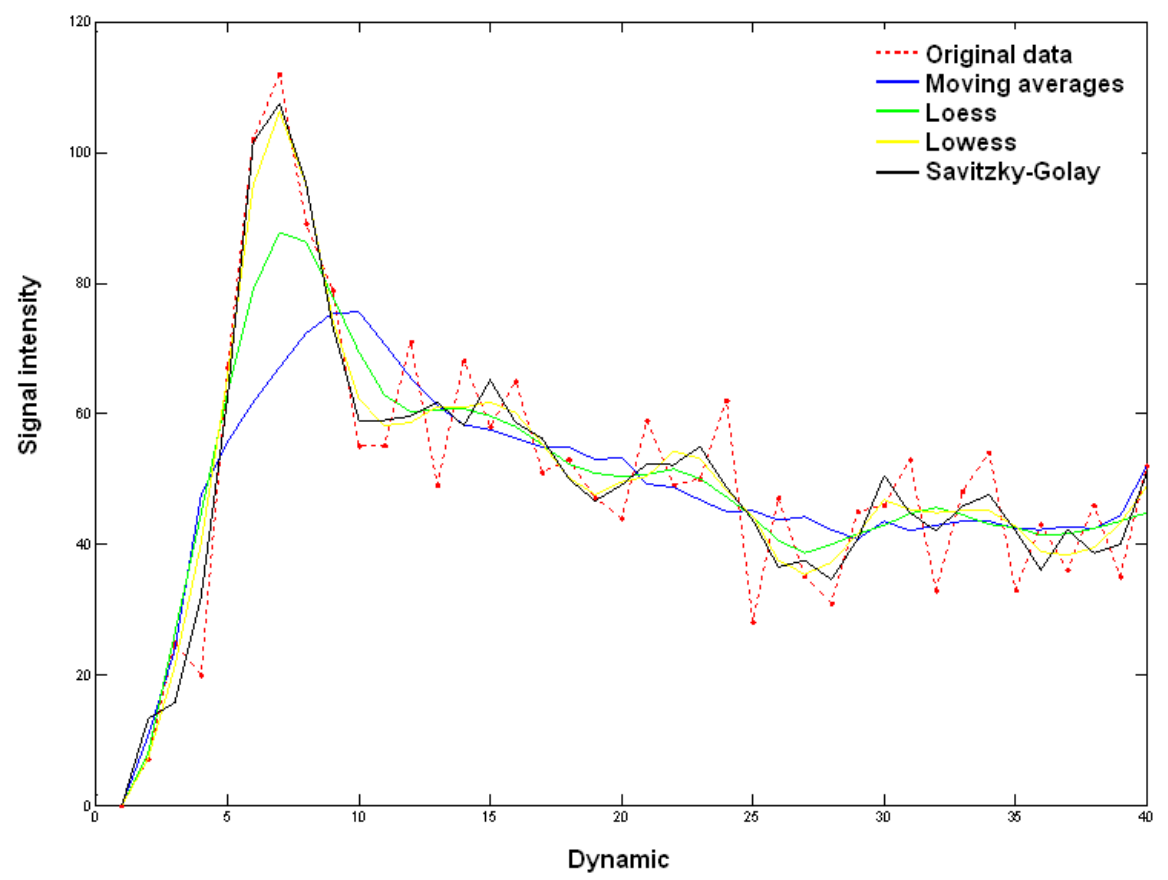

Figure 2.4. Examples of temporal filters. It can be observed that moving averages provides good oscillation reduction but also loses the information of the peak. On the other hand, LOESS, LOWESS and Savitzky-Golay preseve more information of the peak. LOWESS and SavitzkyGolay preserve it much better, but fail in reducing the oscillations as effectively as LOESS.

As a methodological improvement, a modification of the LOESS filter was developed by studying the filtered curve gradient. When this gradient showed high results (a value of the gradient higher than the mean gradient plus 3 standard deviations), the original curve values were used instead of the filtered ones, therefore maintaining the arterial peak exactly at the original magnitude and dynamic, preserving the smoothing of the rest of the curve (figure 2.5). 


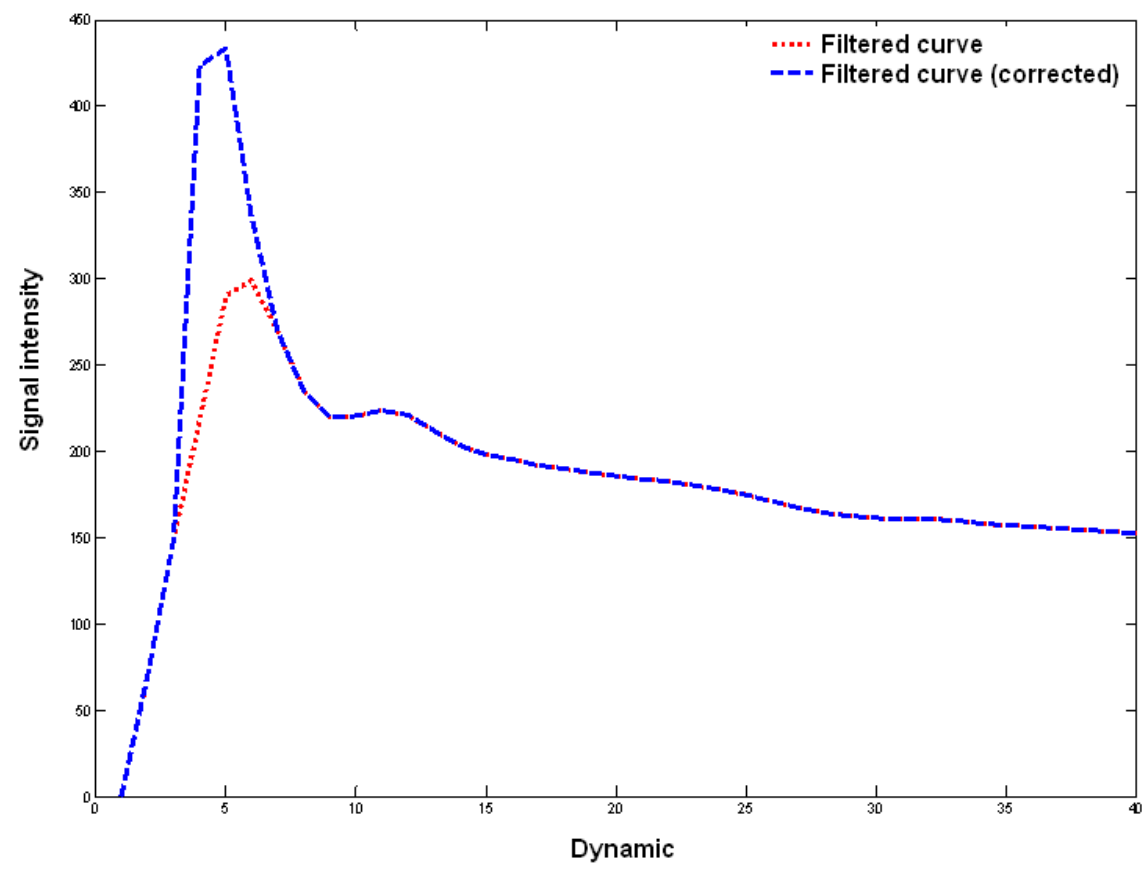

Figure 2.5. Correction of the LOESS filtered curve with the original peak points.

\subsubsection{Image registration}

Image registration can be defined as the process of transforming different images (from one or several imaging modalities) into a common set of coordinates, so that a pixel can be univocally identified through the whole set of images (i.e. through different modalities or in one modality throughout time). The registration problem can be formally expressed as:

$$
T: p_{A} \rightarrow p_{B} \Leftrightarrow T\left(p_{A}\right)=p_{B}
$$

where $T$ denotes a spatial transform that maps coordinates (spatial locations) from one image or coordinate space to another image or coordinate space, $p_{A}$ and $p_{B}$ denote coordinate points (pixel locations) in images $A$ and $B$, respectively. The domain where $\mathrm{T}$ is defined is called the search space of the registration problem. The function that defines the quality factor for the registration result is called the 
registration metric. The algorithm that maximizes the chosen metric is called search strategy. Therefore, the registration problem can be put as the maximization (or minimization) of a function that compares two (sets of) images. One of the most common applications of image registration is the correction of patient's movement in MR perfusion studies, where acquisition takes place during several minutes. This movement is usually related to breathing or other involuntary actions.

Breathing movement is especially important in abdominal studies (such as liver). Although patients are normally instructed to hold breathing during the acquisition or to breathe smoothly, there is always a certain amount of movement that cannot be eliminated during acquisition. New software and hardware developments are being introduced which help to acquire better images [94], but it is still necessary to use post-processing tools to obtain more accurate results. In the abdomen, breathing artifacts take place mostly in updown direction, because of the diaphragm movement (figure 2.6).

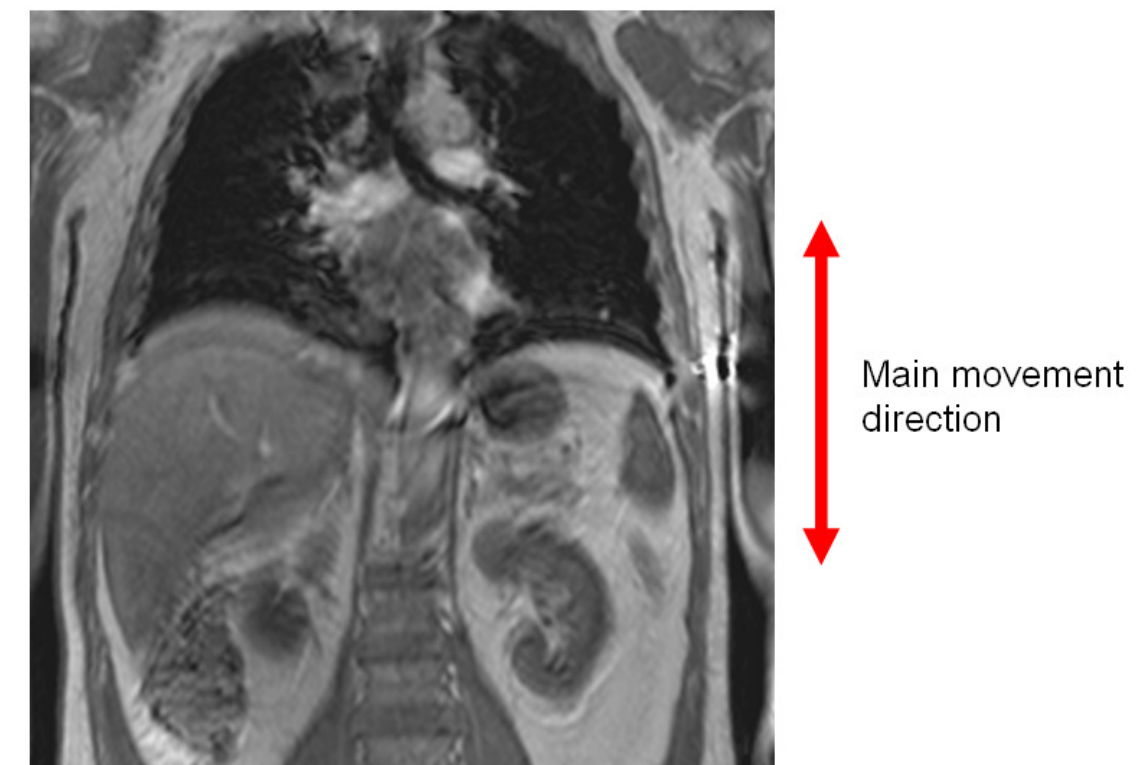

Figure 2.6. Coronal view of thorax and abdomen with the direction in which most of the movement occurs. Note the diaphragm movement related ghost artifacts. 


\section{$\underline{\text { Registration algorithms }}$}

Depending on the application, several classifications can be used [95]:

- Dimensionality: 2D/2D, 2D/3D, 3D/3D.

- Nature of registration basis:

○ Extrinsic: invasive (stereotatic frames) or non-invasive (moulds, frames).

○ Intrinsic: landmark based (anatomical) or segmentation based (points, curves, surfaces, active contours).

○ Non-image based: calibrated coordinate systems.

- Nature of transformation:

○ Linear: rigid (translation, rotation and scaling) or affine (mapping parallel lines) transforms.

○ Elastic or non-rigid: image warping.

- Domain of the transformation: local or global.

- Interaction: automatic, semi-automatic (initialization and check points) or interactive.

- Modalities involved: monomial or multimodal.

- Subject: intrasubject, intersubject or atlas-based.

- Image properties: intensity-based or feature-based.

- Image resolution: multi-resolution (coarse to fine) or mono-resolution.

\section{$\underline{\text { Similarity metrics }}$}

To specify whether two images are correctly aligned it is necessary to use appropriate similarity metrics. These metrics are usually based on corresponding points and their image intensities [96].

As it is not the aim of this thesis to review registration algorithms thoroughly, two of the most common metrics are introduced: 
Absolute difference between images (mean square difference):

$$
M(A, B)=\sqrt{\sum_{i, j \in S}\left(a_{i}-b_{j}\right)^{2}}
$$

where $(i, j)$ denotes a couple of corresponding voxels from each image and $S$ is the domain where the images are defined. This approach is very effective when the images to be registered are quite similar.

\section{Mutual information}

Mutual information $(M I)$ is a concept from information theory [97] which measures the dependence between two variables, so that if two variables are independent, $M I$ equals zero. It is related to the image entropy by:

$$
M I(X ; Y)=H(X)+H(Y)-H(X, Y)
$$

where $X$ and $Y$ are the two images and $H$ is the entropy of a random variable:

$$
H(X)=-\sum_{x_{i} \in \Omega_{X}} \log \left[P\left(X=x_{i}\right)\right] \cdot P\left(X=x_{i}\right)
$$

and the joint entropy:

$$
H(X ; Y)=-\sum_{x_{i} \in \Omega_{X}} \sum_{y_{j} \in \Omega_{Y}} \log \left[P\left(X=x_{i}, Y=y_{j}\right)\right] \cdot P\left(X=x_{i}, Y=y_{j}\right)
$$

The $M I$ criterion is met when the intensity values of corresponding voxels is maximal, which is directly related to the geometrical alignment of the 
images. As many optimization algorithms are formulated as minimization functions, the $M I$ is often used in its negative form.

\section{$\underline{\text { Practical case: liver registration }}$}

From the applications presented in this thesis (OHSS characterization, cartilage degeneration assessment, grade-IV CNS astroyctomas and HCC), the liver was the only organ which needed registration. As shown above, liver is mainly affected by head-feet movement, although a minor deformation of the organ also occurs.

Two approaches were considered:

\section{Rigid registration}

A series of rectangular regions were placed on the interface between the liver and the right lung. These rectangles were propagated through all the dynamics and changes of intensity were assessed to account for liver movement (figure 2.7). When the amount of black intensity decreased it meant that liver moved upwards (less lung voxels in the rectangle), while when the amount of black intensity increased it meant that liver moved downwards (more lung voxels in the rectangle). It is important to notice that lung intensity must be taken as reference because in a perfusion study the liver enhances, while the lung remains hypointense.

With this approach it is possible to obtain a good estimation of the headfeet movement of the liver, which can be corrected by applying the opposite displacement with a column-based correction (figure 2.7). Although fairly simple, this approach is fast and offers good results. 


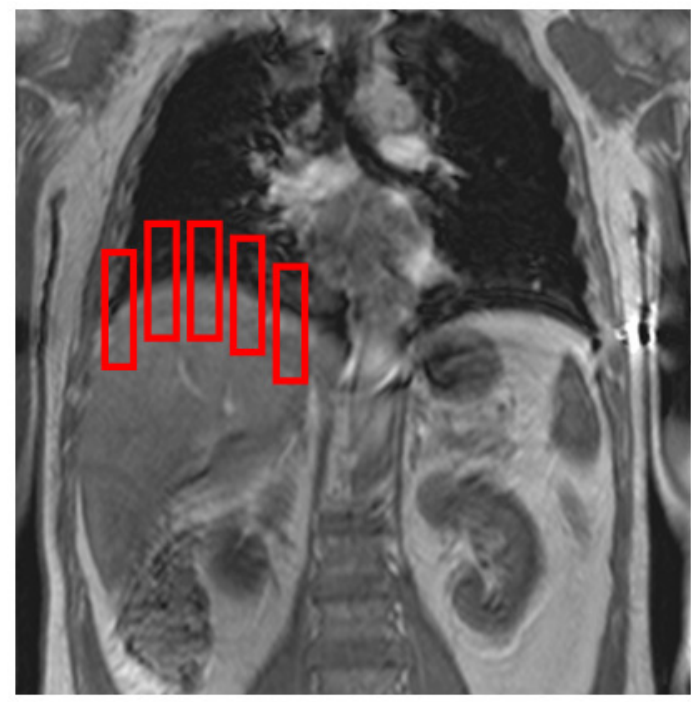

a

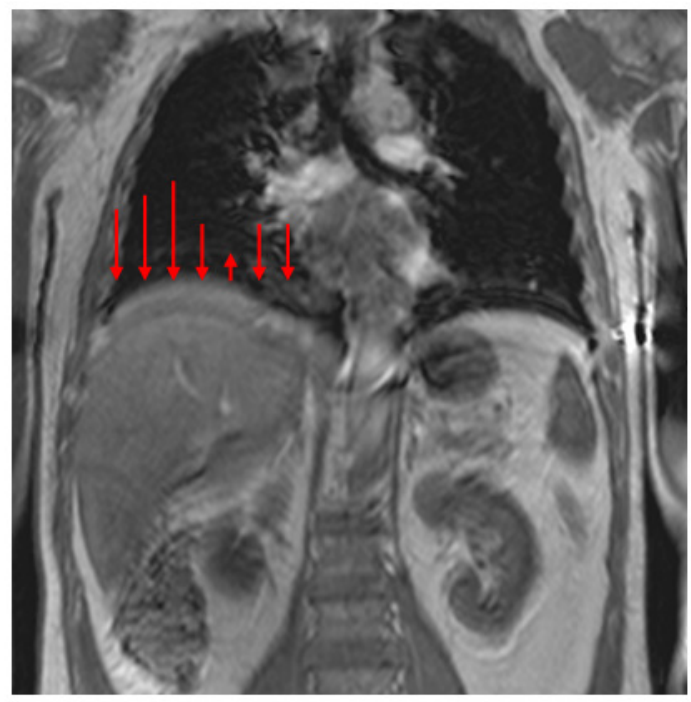

b

Figure 2.7. Bottom-up registration with the use of navigating interfaces (a). These interfaces calculate the amount of bottom-up movement by observing the oscillations throughout the whole dynamic study. Then a different bottom-up correction can be applied column by column for each dynamic (b).

\section{Non-rigid registration}

For ease of use, the Matlab package SPM (www.fil.ion.ucl.ac.uk/spm) was used to test non-rigid registration on liver images. SPM uses discrete cosine transform (DCT) as the transformation model and the sum of squared differences between an image and a template as the similarity measure [98]. Before this non-linear transform (or warping), a linear transform (affine) is also applied. Then, the non-linear warps are modeled by a linear combination of discrete cosine-basis functions with low-spatial frequency to reduce the number of parameters to be fitted [99].

The registered images showed qualitatively less movement and the extracted curves had fewer oscillations (figure 2.8). However, this registration strategy has important time limitations, as the computational burden is very high for the degree of improvement. 


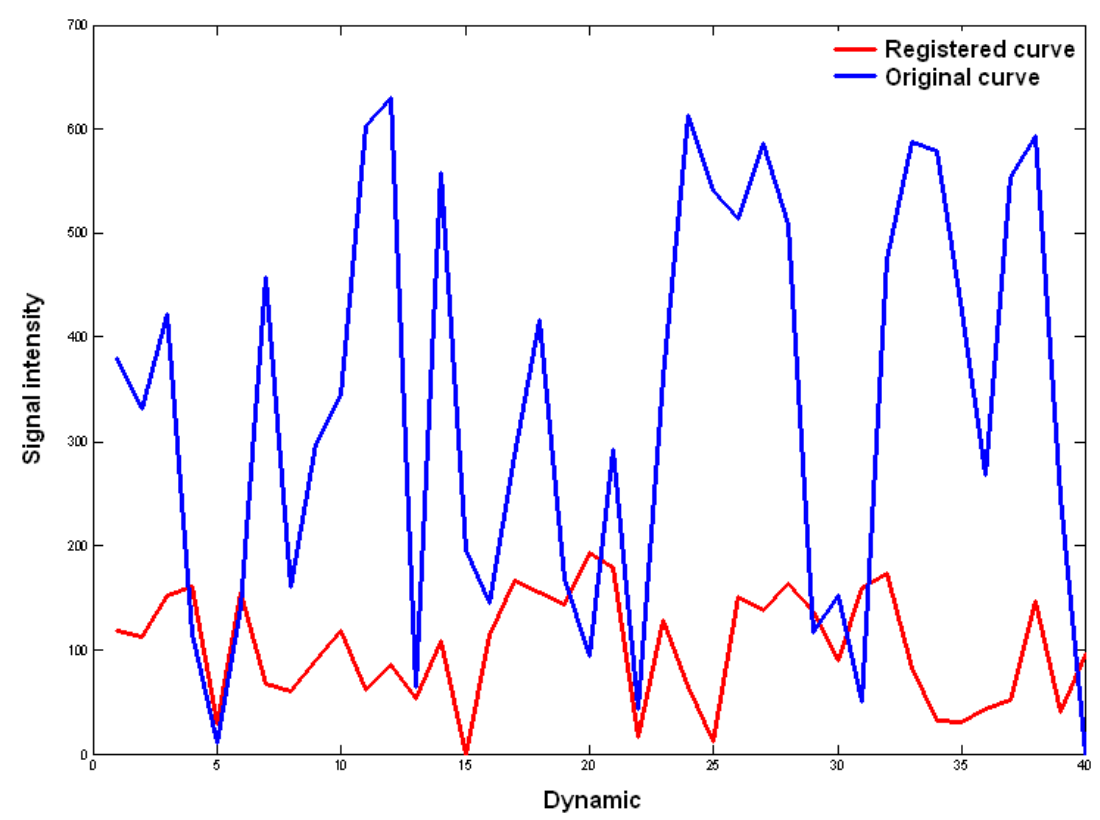

Figure 2.8. Comparison of the oscillation of a voxel in the interface liver-lung before and after applying non-rigid image registration. It can be seen that the oscillations are much more reduced after registration. The fact that the intensity is much lower afterwards is explained because the observed voxel was mainly placed at the lung.

\section{Adopted solution}

After multiple tests, a conclusion is drawn from the application of registration to liver perfusion studies. Although spatial registration is essential in some applications in order to obtain accurate pixel-based analysis, in PK modeling it is more important to extract good enhancement curves (with low oscillations) for the fitting algorithms. These two characteristics are closely related, but as it has been shown in the temporal filters section, it is possible to obtain reasonably good enhancement curves without the need to apply other improvement tools.

Therefore, only in those cases where movement is substantially relevant a registration is applied, combining both rigid and non-rigid approaches. Otherwise smoothing temporal filters are used to improve enhancement curves. 


\subsection{Application of PK modeling}

\subsubsection{Study cases}

Regarding the clinical applications presented in the introduction chapter, the particular methodology for each case will be described herein. Subsections 2.2.1.1, 2.2.1.2, 2.2.1.4 and 2.2.1.6 have been directly adapted from publications [100-103], respectively.

\subsubsection{Evaluation of ovarian hyperstimulation syndrome (OHSS) treatment}

\section{Description}

Evaluation of the microvascular effects of cabergoline treatment in women with OHSS.

\section{Hypothesis}

The differences in the PK parameters between the patients treated with cabergoline and the patients treated with placebo will demonstrate the effect of the drug as repressor of the OHSS. It is expected that treated patients will show lower values of $K^{\text {trans }}$ and $v_{e}$. This study will objectively quantify the process of angiogenesis which takes places during ovarian stimulation.

\section{Study group}

20 young women were randomly separated into two groups: 10 treated with cabergoline ( $29 \pm 3$ years old) and 10 with placebo $(31 \pm 7$ years old). All of them were ovary donors with a high risk of developing OHSS, as they showed OHSS antecedents or a high response in a previous cycle of controled ovarian hyperstimulation. 


\section{$\underline{\text { Image acquisition }}$}

All patients underwent two DCE-MR studies, the first before ovarian stimulation with hCG and the second five days after hCG administration. The acquisition parameters are shown in table 2.1.

\begin{tabular}{cc}
\hline & PK study \\
\hline Magnetic field & $1.5 \mathrm{~T}$ \\
Sequence & T1w sGRE \\
Orientation & Transversal \\
Fat suppression & Yes \\
Repetition time & $71.3 \mathrm{~ms}$ \\
Echo time & $1.13 \mathrm{~ms}$ \\
Flip angle & $60^{\circ}$ \\
Slices & 20 \\
Voxel size & $1.83 \times 2.80 \times 6 \mathrm{~mm}$ \\
Dynamics & 50 \\
Temporal resolution & $4.4 \mathrm{~s}$ \\
Acquisition time & $5 \mathrm{~min} 35 \mathrm{~s}$ \\
Contrast dose & $0.2 \mathrm{ml} / \mathrm{kg} @ 4 \mathrm{ml} / \mathrm{s}$ \\
\hline Table 2.1. Sequence parameters for the OHSS assessment.
\end{tabular}

\section{$\underline{\text { Image analysis }}$}

The arterial input function (AIF) was manually selected with a region of interest (ROI) over the iliac artery. It was made sure that all AIFs had the usual shape: base zone, fast enhancing peak, fast washout and slight recirculation. The ROIs for the ovaries were also manually selected, taking special care to select stroma alone. Enhancement curves were then extracted pixel by pixel (figure 2.9). 


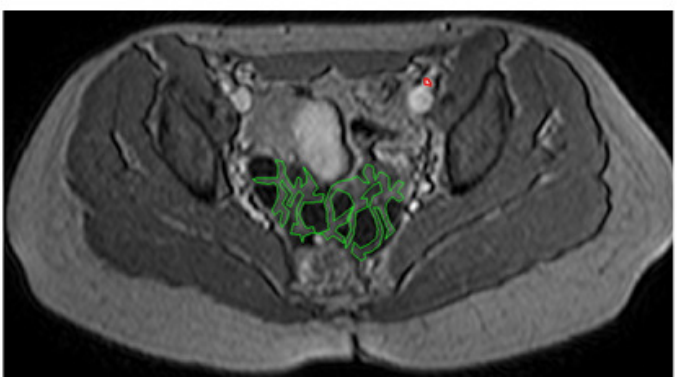

(a)

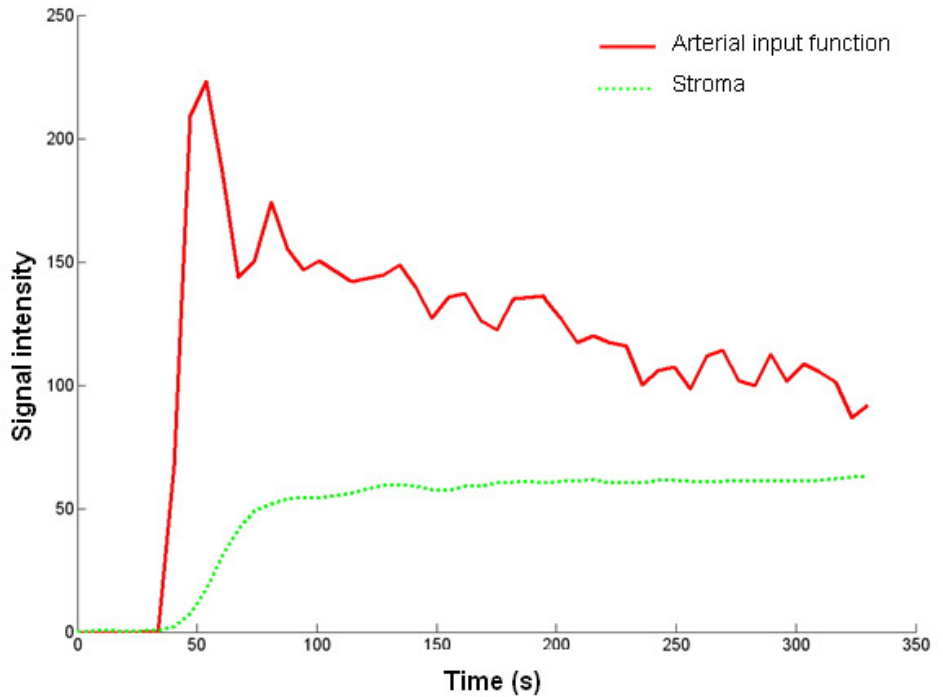

(b)

Figure 2.9. (a) Selection of the iliac artery and the ovary stroma as arterial input function and tissue for extraction of signal intensity curves (b).

\section{Intensity to concentration conversion}

Due to study design decisions, the acquisition sequence for T1 mapping was not included in the PK MR protocol at that time. It has been already introduced that when using spoiled gradient echo sequences, pre-contrast T1 values are necessary in order to convert intensity into contrast concentration values accurately, but for this study a polynomial conversion was used:

$$
C(t)=5.839 \cdot 10^{-8} \cdot s(t)^{3}-5.670 \cdot 10^{-5} \cdot s(t)^{2}+1.793 \cdot s(t)-1.254
$$


where $s(t)$ is the normalized signal intensity. This equation was obtained from a phantom study with thirty-one tubes of contrast dilution at different concentrations (figure 2.10).

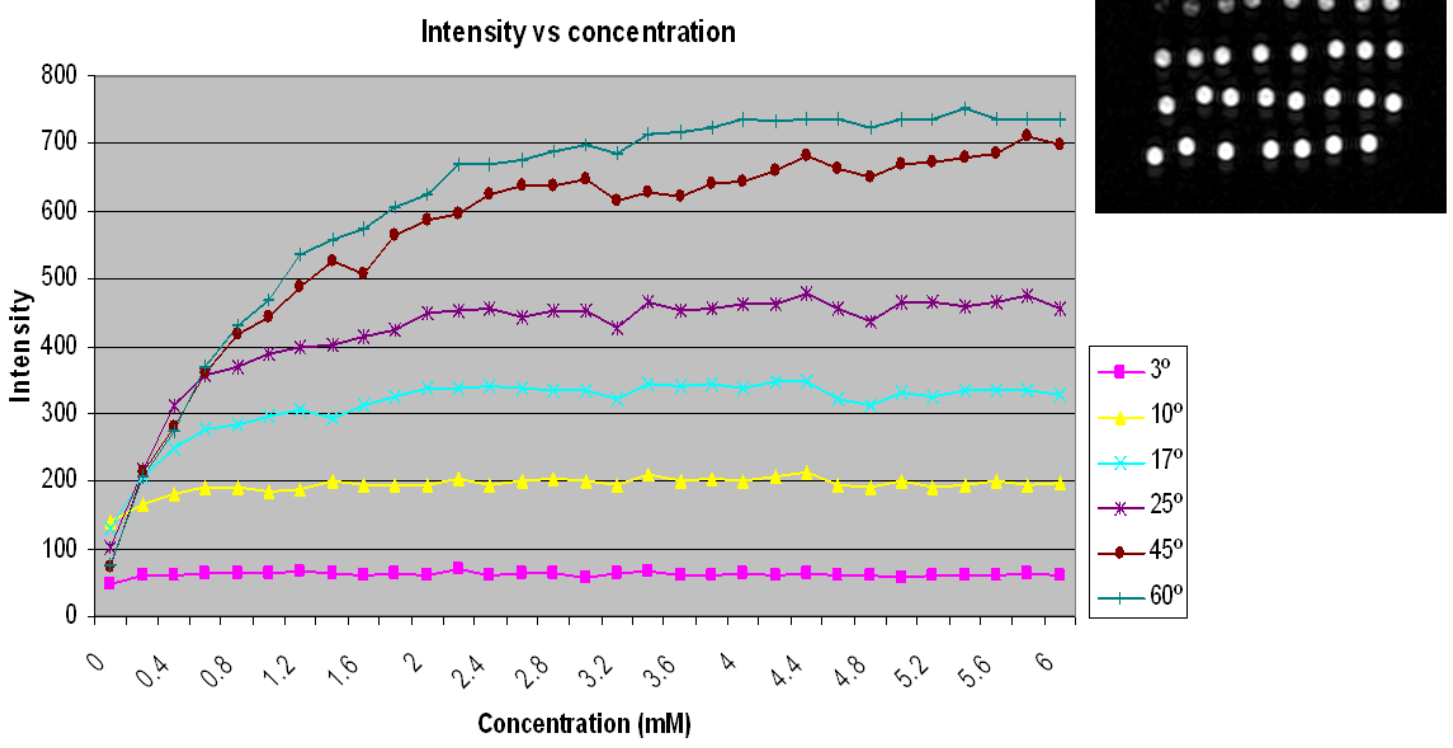

Figure 2.10. Graph showing the variation of the signal intensity as a function of the concentration for different flip angles, obtained from thirty-one tubes prepared at different concentrations (top right image).

\section{PK modeling}

Ovarian stroma was initially considered as a highly vascular tissue, so a oneinput two-compartment model was considered to be the best option:

$$
C_{O}=v_{p} \cdot C_{a}(t)+\int_{0}^{t} K^{\text {trans }} \cdot C_{a}(u) \cdot e^{-k_{e p}(u-t)} d u
$$

where $C_{O}$ is the concentration in the stroma, $C_{a}$ is the concentration in the artery and $K^{\text {trans }}, k_{e p}$ and $v_{p}$ are the PK parameters. Interstitial space is obtained as $v_{e}=K^{\text {trans }} / k_{e p}$. 
However, for comparison purposes, a one-input one-compartment model was also used:

$$
C_{O}=\int_{0}^{t} K^{\text {trans }} \cdot C_{a}(u) \cdot e^{-k_{e p}(u-t)} d u
$$

The Levenberg-Marquardt least mean squares algorithm [83] was used for curve fitting.

\section{$\underline{\text { Statistical analysis }}$}

The differences between both groups (treatment and placebo) for the two MR studies and for each PK model were assessed with a $t$ test for independent variables. A p-value $<0.05$ was considered as statistically significant.

The intraclass correlation coefficient (ICC) was used to assess methodological reproducibility by repeating the acquisition and the analysis for both PK models in 10 randomly selected studies, with a lapse of one week between analyses. A value of ICC $>0.75$ was considered as good reproducibility [104].

\subsubsection{Assessment of cartilage degeneration in $1.5 \mathrm{~T}$}

\section{Description}

To study the neovascularization processes associated to the degeneration of the patellar cartilage by means of PK modeling.

\section{Hypothesis}

The PK modeling of the patellar cartilage will allow differentiating normal from pathological conditions. 


\section{Study group}

22 subjects underwent 1.5T DCE-MRI of the knee (5 healthy, 8 with chondromalacia and 9 with osteoarthritis). Healthy subjects and patients with chondromalacia were similar in age $(43.6 \pm 15$ and $33.3 \pm 11.8$ years old, respectively), but younger than patients with osteoarthritis (58.9 \pm 11.5 years old) $(\mathrm{p}=0.002)$. Regarding gender, there were 14 women (3 normal, 7 with chondromalacia and 4 with osteoarthritis) and 8 men (2 normal, 1 with chondromalacia and 5 with osteoarthritis).

\section{$\underline{\text { Image acquisition }}$}

The acquisition parameters that were used are shown in table 2.2.

\begin{tabular}{cc}
\hline & PK study \\
\hline Magnetic field & $1.5 \mathrm{~T}$ \\
Sequence & T1w sGRE \\
Coil & Surface coil \\
Orientation & Transversal \\
Fat suppression & Yes \\
Repetition time & $3.47 \mathrm{~ms}$ \\
Echo time & $1.93 \mathrm{~ms}$ \\
Flip angle & $10^{\circ}$ \\
Slices & 10 \\
Acquired voxel size & 50 \\
Dynamics & $2.9 \mathrm{~s}$ \\
Temporal resolution & $2 \mathrm{~min} 25 \mathrm{~s}$ \\
Acquisition time & $0.78 \mathrm{ml} / \mathrm{kg} @ 4 \mathrm{ml} / \mathrm{s}$ \\
Contrast dose & (Omniscan, GE Healthcare, UK) \\
\hline Table 2.2. Sequence parameters for the cartilage assessment in $1.5 \mathrm{~T}$.
\end{tabular}




\section{$\underline{\text { Image analysis }}$}

A ROI containing the cartilage was defined by manually drawing the cartilage in the pre-contrast image of the transverse slice through the largest left-right patellar diameter. The AIF was taken from a ROI drawn within the popliteal artery (figure 2.11). All the pixels within the arterial ROI were averaged at every time point. No further function, apart from pixel averaging and moving averages, was used to smooth the contrast enhanced curves. As the knee was tightly immobilized, image registration was not considered necessary. All intensity curves were normalized before intensity to concentration conversion.

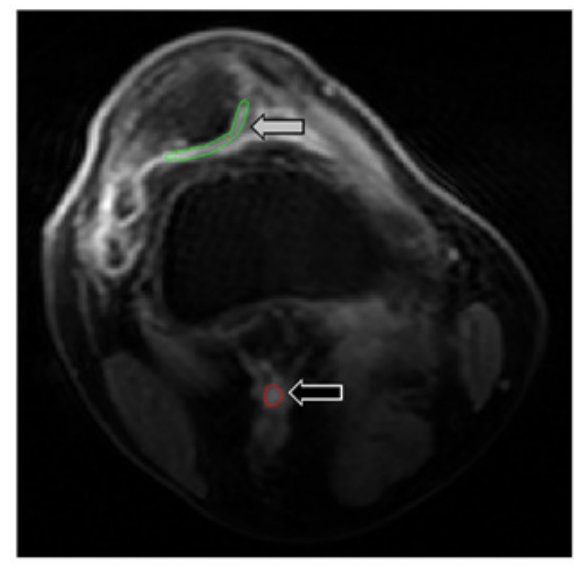

a

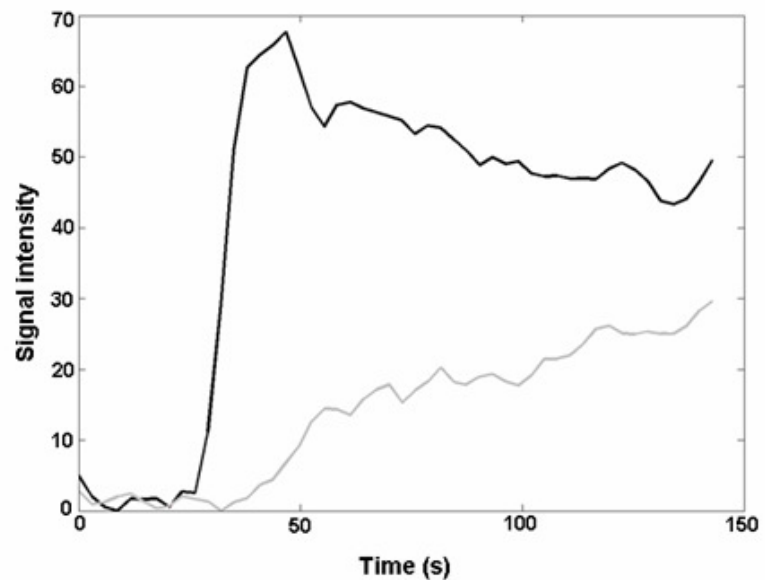

b

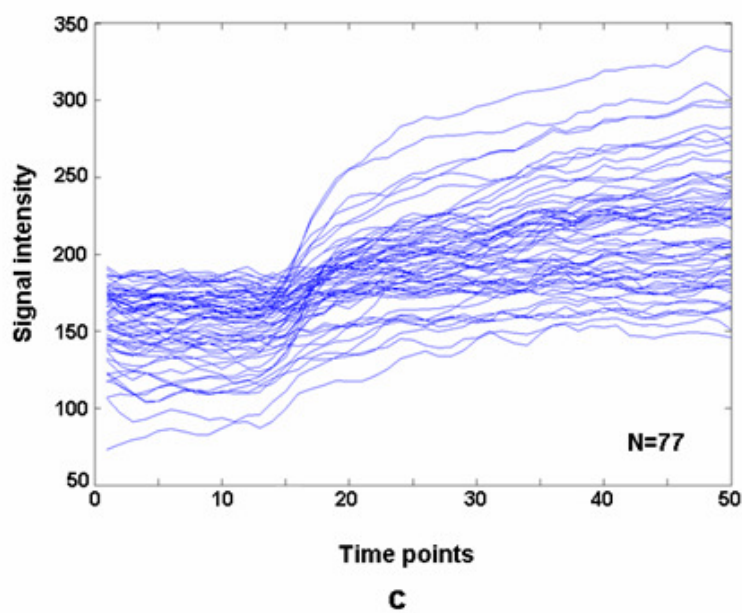

Figure 2.11. (a) DCE-MRI slice showing the regions of interest for the cartilage (gray arrow) and the popliteal artery (black arrow). (b) Signal enhancement for the artery (black) and the whole cartilage (gray). (c) Signal enhancement for all the voxels pertaining to the cartilage ROI. 


\section{Intensity to concentration conversion}

To convert the intensity vs. time curves into concentration vs. time curves, it was assumed that the normalized intensity vs. time curve can be linearly related to the contrast agent concentration [17]:

$$
\frac{S_{t}(t)}{S_{0}}-1 \approx T 1_{0} \cdot r_{1} \cdot C_{t}(t)
$$

where $S_{t}(t)$ is the intensity vs. time curve, $S_{0}$ is the signal intensity before contrast administration, $T 1_{0}$ is the $T 1$ before contrast administration, and $r_{1}$ is the longitudinal relaxivity of the contrast agent. This approach is derived from the assumption that $T R \times r_{1} \times C_{t}<<1, T E \times r_{2} \times C_{t}<<1$ and $T R<<T 1_{0}$ (where $r_{2}$ is the transversal relaxivity of the contrast agent). This approach is valid in this study, assuming a maximum weight of $100 \mathrm{~kg}$ for a patient:

- $T R \times r_{1} \times C_{t} \leq 3.47 \mathrm{~ms} \times 4.5 \mathrm{mM}^{-1} \mathrm{~s}^{-1} \times 0.2 \mathrm{ml} / \mathrm{kg} \times 100 \mathrm{~kg} \times 0.5 \mathrm{mM} / \mathrm{ml}=0.2$ $<<1$ (assuming maximum concentration).

- $T E \times r_{2} \times C_{t} \leq 1.93 \mathrm{~ms} \times 5.5 \mathrm{mM}^{-1} \mathrm{~s}^{-1} \times 0.2 \mathrm{ml} / \mathrm{kg} \times 100 \mathrm{~kg} \times 0.5 \mathrm{mM} / \mathrm{ml}=0.1$ $<<1$ (assuming maximum concentration).

- $T R<<T 1_{0} \approx 1 \mathrm{~s}[17,105]$.

\section{PK modeling}

In this study one-input and both one- and two-compartment models were used (the expressions for these models can be found in the previous application).

Although the one-input one-compartment may produce an overestimation of the parameters $K^{\text {trans }}$ and $v_{e}[12,106]$, it may be appropriate in those situations where the vascular contribution may be neglected, as in normal or weakly irrigated tissues like the cartilage [4]. To assess these differences, both models were used and compared. 
Only pixels which enhanced, defining enhancement as 3 standard deviations higher than the base image before contrast administration, were computed in the PK analysis. Enhanced pixels were sieved, and those with $v_{e}$ values higher than $100 \%$ were discharged.

The Levenberg-Marquardt least mean squares algorithm [85] was used for curve fitting.

\section{$\underline{\text { Statistical analysis }}$}

A one-way analysis of variance (ANOVA) was performed with a $p$-value $<0.05$ considered to be statistically significant. For the test-retest analysis of variability the root mean square (RMS) coefficient of variation was calculated:

$$
R M S_{-} C o V=\sqrt{\frac{\sum_{i=1}^{N}\left(\frac{\sigma_{i}}{\mu_{i}}\right)^{2}}{N}}
$$

where $\sigma_{i}$ is the standard deviation, $\mu_{i}$ the mean of each pair of measurements and $N=10$ cases. The 10 patients selected for the variability analysis were randomly chosen (2 normal, 3 with chondromalacia and 5 with osteoarthritis). Lower values of RMS CoV correspond to a higher reproducibility of the results.

\subsubsection{Assessment of femoropatellar cartilage degeneration in $3.0 \mathrm{~T}$}

\section{Description}

Cartilage degeneration is associated with hypoxia, inflammation and neoangiogenesis, expressed as an increase of VEGF production in the cartilage matrix. The purpose of this work is to study the PK parameters derived from the analysis of 3T DCE-MR images of the femoral and patellar cartilages and their relationship with the radiological diagnosis. 


\section{$\underline{\text { Hypothesis }}$}

The PK modeling of the patellar cartilage will also allow differentiating normal from pathological conditions in $3.0 \mathrm{~T}$.

\section{Study group}

54 patients with different cartilage conditions (normal, initial degeneration and advanced degeneration) were included in the study. Cartilages were analyzed separately (patellar and femoral) and they were classified by an experienced radiologist as normal (23), with initial degeneration (23) and with advanced degeneration (8).

\section{Image acquisition}

Along with the conventional knee protocol, the acquired sequences are shown in table 2.3.

\begin{tabular}{ccc}
\hline & T1 mapping & PK study \\
\hline Magnetic field & $3.0 \mathrm{~T}$ & $3.0 \mathrm{~T}$ \\
Sequence & T1w sGRE & T1w sGRE \\
Orientation & Sagittal & Sagittal \\
Fat suppression & No & Yes \\
Repetition time & $9.2 \mathrm{~ms}$ & $3.48 \mathrm{~ms}$ \\
Echo time & $5.4 \mathrm{~ms}$ & $1.86 \mathrm{~ms}$ \\
Flip angle & $2^{\circ}, 5^{\circ}, 7^{\circ}, 10^{\circ}, 15^{\circ}, 20^{\circ}, 25^{\circ}, 30^{\circ}, 40^{\circ}, 60^{\circ}$ & $10^{\circ}$ \\
Slices & 10 & 10 \\
Voxel size & $0.78 \times 0.78 \times 6 \mathrm{~mm}^{\circ}$ & $0.76 \times 0.76 \times 6 \mathrm{~mm}$ \\
Dynamics & - & 50 \\
Temporal resolution & - & $2.9 \mathrm{~s}$ \\
Acquisition time & $1 \mathrm{~min}$ & $2 \mathrm{~min} 25 \mathrm{~s}$ \\
Contrast dose & - & $($ Dotarem $)$ \\
\hline
\end{tabular}

Table 2.3. Sequence parameters for the cartilage assessment in $3.0 \mathrm{~T}$. 


\section{$\underline{\text { Image analysis }}$}

Cartilages were semi-automatically segmented from a high resolution water selective excitation sequence by using Otsu's multiple thresholding method [107]. Once cartilage was correctly outlined, masking contours were rescaled and applied onto the perfusion and T1 mapping images (figure 2.12). The popliteal artery was manually segmented as arterial input function.
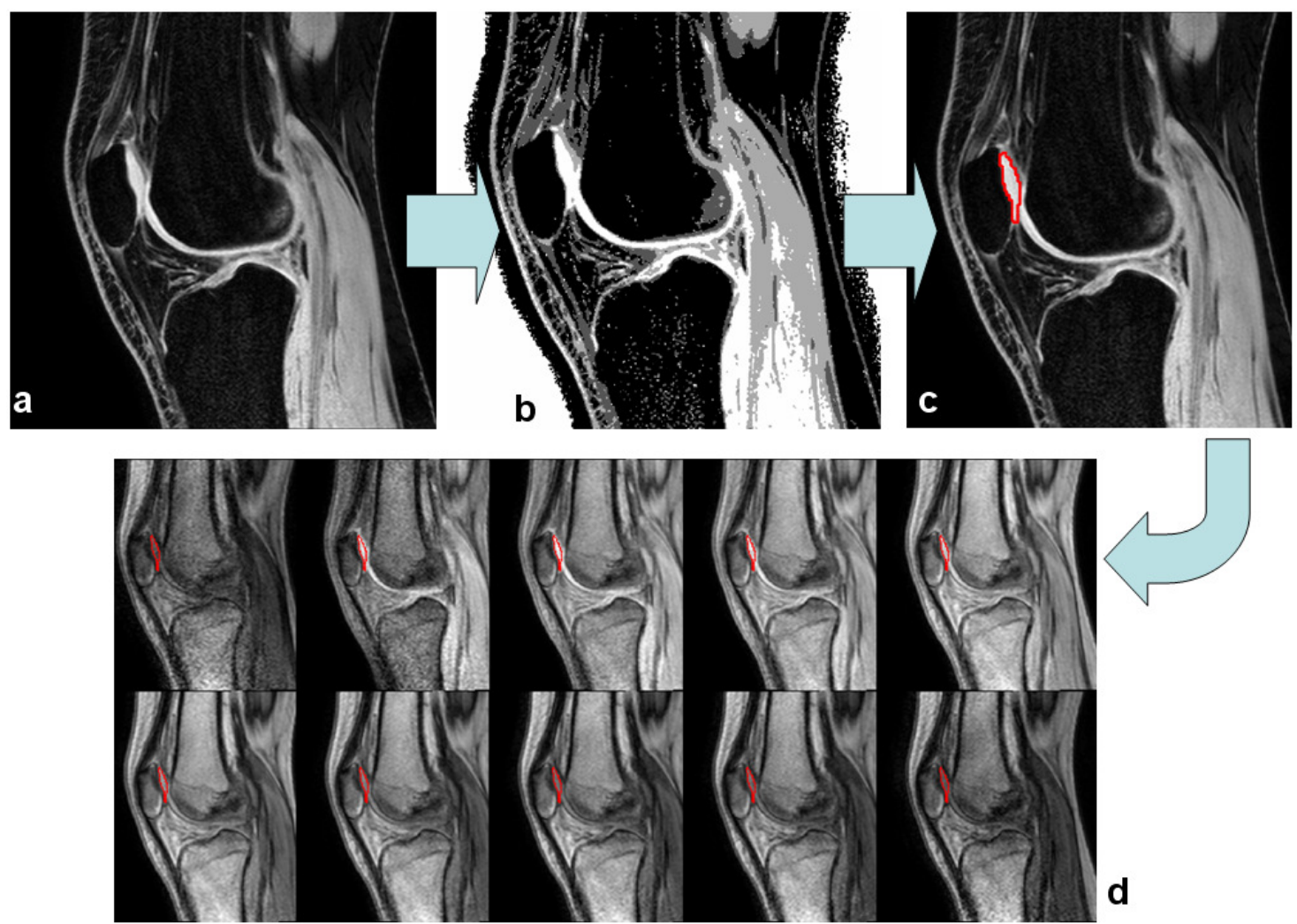

Figure 2.12. Workflow for the segmentation of the patelar cartilage: (a) High contrast image for segmentation, (b) Otsu's thresholding, (c) selection of the patelar cartilage, and (d) propagation of the ROI in the same slice for all the flip angles (T1 mapping sequence).

Native T1 values were also calculated for every voxel with the multiple flip angle sequence and the method described previously. 


\section{Intensity to concentration conversion}

Intensity vs. time curves were converted into concentration vs. time curves by calculating the variation of $T 1$ through time from the 3D-sGRE sequence and subtracting the pre-contrast $T 1$ of the voxel, as described above.

\section{PK modeling}

As the cartilage presents a very low vascular profile, the vascular contribution was dismissed and the one-input one-compartment PK model was used, obtaining the three known parameters: $K^{\text {trans }}, k_{e p}$ and $v_{e}$.

$\underline{\text { Statistical analysis }}$

Differences among the three groups were assessed with an ANOVA and Student-Newman-Keuls post-hoc tests.

\subsubsection{Evaluation of glucosamine sulfate for the osteoarthritis treatment}

\section{Description}

Assessment of the microvascular effects of the glucosamine sulfate on osteoarthritic cartilages.

\section{Hypothesis}

Glucosamine sulfate treatment has an influence over the neovascularity abnormalities of the osteoarthritic cartilage. The PK analysis of the cartilage can be used to evaluate these effects.

\section{Study group}

20 patients with non-advanced degenerative patellar conditions, where surgery was not indicated, were included. All the patients were autonomous for daily life activities and did not need help to stroll. None were under treatment (neither 
corticosteroids nor nutraceuticals) before enrolling in the study. None had rheumatic diseases, severe articular inflammation, neither previous history of significant traumatic knee injury. Four patients were withdrawn after the first MR examination because they failed in therapeutic adherence, so that finally there were 16 patients fulfilling all the study enrolling conditions. They were classified as having chondromalacia (7 cases, $29 \pm 12$ years old, 1 man and 6 women) or osteoarthritis (9 cases, $59 \pm 9$ years old, 5 men and 6 women) by means of MR imaging (signal intensity variations and cartilage surface ulcerations in patients with osteoarthritis), arthroscopy and surgery. Patella surface analysis was performed with the transversal T2-weighted high-resolution images.

Patients were distributed in the glucosamine sulfate ( 3 with chondromalacia and 7 with osteoarthritis) or control (2 with chondromalacia and 4 with osteoarthritis) groups using a randomized list. The groups were not statistically different regarding disease condition, age or sex.

Both groups started treatment immediately after the first MR study. All patients took the medication interruptedly until the second MR examination. The glucosamine group had 1,500 mg (Xicil, Rottapharm-Madaus) orally once a day, while the control group had oral $650 \mathrm{mg}$ of acetaminophen (Dolostop, Bayer) once a day.

\section{Clinical evaluation}

All patients were clinically evaluated twice; the first examination was performed before starting the treatment and the second 6 months later. Two scores were used in both clinical evaluations: the pain visual analog scale $(V A S)$ (range 1-10, best to worse) and the widely used functional outcome American Knee Society $(A K S)$ score (range 1-100, worst to best). The AKS is divided into two parts: the Knee Score, which considers pain, stability and range of motion, with deductions for flexion contractures, extension lag and misalignment; and the Function Score, 
which utilizes walking distance and stair climbing, with deduction for the use of a walking aid [108].

\section{Image acquisition}

The acquisition parameters used for both MR examinations are indicated in table 2.4 .

\begin{tabular}{cc}
\hline & PK study \\
\hline Magnetic field & $1.5 \mathrm{~T}$ \\
Sequence & T1w sGRE \\
Orientation & Transversal \\
Fat suppression & Yes \\
Repetition time & $3.47 \mathrm{~ms}$ \\
Echo time & $1.93 \mathrm{~ms}$ \\
Flip angle & $10^{\circ}$ \\
Slices & 10 \\
Voxel size & $0.78 \times 0.78 \times 10 \mathrm{~mm}$ \\
Dynamics & 50 \\
Temporal resolution & $2.9 \mathrm{~s}$ \\
Acquisition time & $2 \mathrm{~min} 25 \mathrm{~s}$ \\
Contrast dose & $0.2 \mathrm{ml} / \mathrm{kg} @ 4 \mathrm{ml} / \mathrm{s}$ \\
\end{tabular}

Table 2.4. Sequence parameters for the treatment assessment.

Image analysis

The same image analysis strategy as in the previous study case was used.

\section{Intensity to concentration conversion}

To convert the intensity vs. time curves into concentration vs. time curves, it was assumed that the normalized intensity vs. time curve was linearly related to the contrast agent concentration [17], as explained in the previous study case. 


\section{PK modeling}

In this study a one-input and one-compartment model was used (the expression for this model can be found in the previous cartilage application, 2.2.1.3).

\section{$\underline{\text { Statistical analysis }}$}

A t-test for independent data was used to evaluate differences (glucosamine vs. controls, expressed in percentage of change) after evaluating the equality of the variance with Levene's test. A p-value<0.05 was considered statistically significant. Calculations of power statistics were also performed for variables showing statistical differences and the curves for power vs. difference in means were obtained.

\subsubsection{Characterization of grade-IV astrocytomas}

\section{Description}

To characterize tumor, peritumoral and normal brain areas with all the modelderived quantitative parameters from MR T2*-enhanced perfusion images.

\section{Hypothesis}

The combination of quantitative parameters obtained from different perfusion models and the use of histograms will provide a better classification of brain regions (tumor, peritumoral and normal).

\section{Study group}

15 patients ( 11 men and 4 women, $58 \pm 13$ years old) with grade-IV astrocytoma (glioblastoma) were included in the study. None had received oncological treatment or surgery before MR imaging. The anatomopathological diagnose was obtained by biopsy in 3 patients and by surgical resection in 12 patients. Tumors were located at the parietal (2), temporal (8) and frontal (5) regions. 


\section{$\underline{\text { Image acquisition }}$}

The acquisition parameters are displayed in table 2.5.

\begin{tabular}{cc}
\hline & PK study \\
\hline Magnetic field & $1.5 \mathrm{~T}$ \\
Sequence & $\mathrm{T} 2^{*}$-w EPI GRE \\
Orientation & Transversal \\
Fat supresión & Yes \\
Repetition time & $836 \mathrm{~ms}$ \\
Echo time & $30 \mathrm{~ms}$ \\
Flip angle & $40^{\circ}$ \\
Slices & 20 \\
Voxel size & $1.8 \times 1.8 \times 7 \mathrm{~mm}$ \\
Dynamics & 40 \\
Temporal resolution & $2.4 \mathrm{~s}$ \\
Acquisition time & $1 \mathrm{~min} 36 \mathrm{~s}$ \\
Contrast dose & $0.2 \mathrm{ml} / \mathrm{kg} @ 5 \mathrm{ml} / \mathrm{s}$ \\
& (Omniscan, GE Healthcare, UK) \\
\hline Table 2.5. Brain perfusion sequence parameters.
\end{tabular}

\section{$\underline{\text { Image analysis }}$}

MR perfusion studies were globally quantified in all the slices where the lesion was visible. The middle cerebral artery was chosen as arterial input function in all the studies. The ROIs corresponding to the tumoral area, the peritumoral area and the contralateral normal brain area were manually selected for each slice. The tumoral ROI was defined as the area with signal decrease after contrast injection. The peritumoral ROI was defined as the area corresponding to $1 \mathrm{~cm}$ surrounding the nodule from the enhancing border and with $T 2^{*}$ signal intensity alterations [109].

All ROIs were selected after consensus by two experienced radiologists. When drawing the areas, special care was taken to exclude vessels which could 
interfere with the perfusion results. Also, when selecting the normal area, it was taken into account that it was not connected to the tumor by white matter tracts. Enhancement curves were extracted pixel by pixel in order to analyze the histograms afterwards.

\section{Intensity to concentration conversion}

To convert signal intensity to concentration vs. time curves, the following equation was used [110]:

$$
C=-\frac{1}{T E} \cdot \log \left(\frac{S}{S_{0}}\right)
$$

where $C$ is the contrast concentration, $S$ is the signal intensity and $S_{0}$ is the signal intensity before contrast injection. It can be seen that this expression is different from that used in spoiled $\mathrm{T} 1 \mathrm{w}$ gradient-echo sequences, as one of the characteristics of T2*-weighted dynamic studies is the decay of the signal as the contrast concentration increases.

\section{PK modeling}

In this study case, three models were used to quantify brain perfusion: singlecompartment, multiple-compartment and first-pass multiple-compartment.

\section{Single-compartment}

The single-compartment (not to be confused with the PK onecompartment model used in previous study cases) is based on the intravascular dilution of the contrast agent. It neglects both tracer recirculation and extravasation. Therefore, to obtain absolute values, it was necessary to correct the extracted curves with a function that estimated a theoretical curve without recirculation (figure 2.13). 


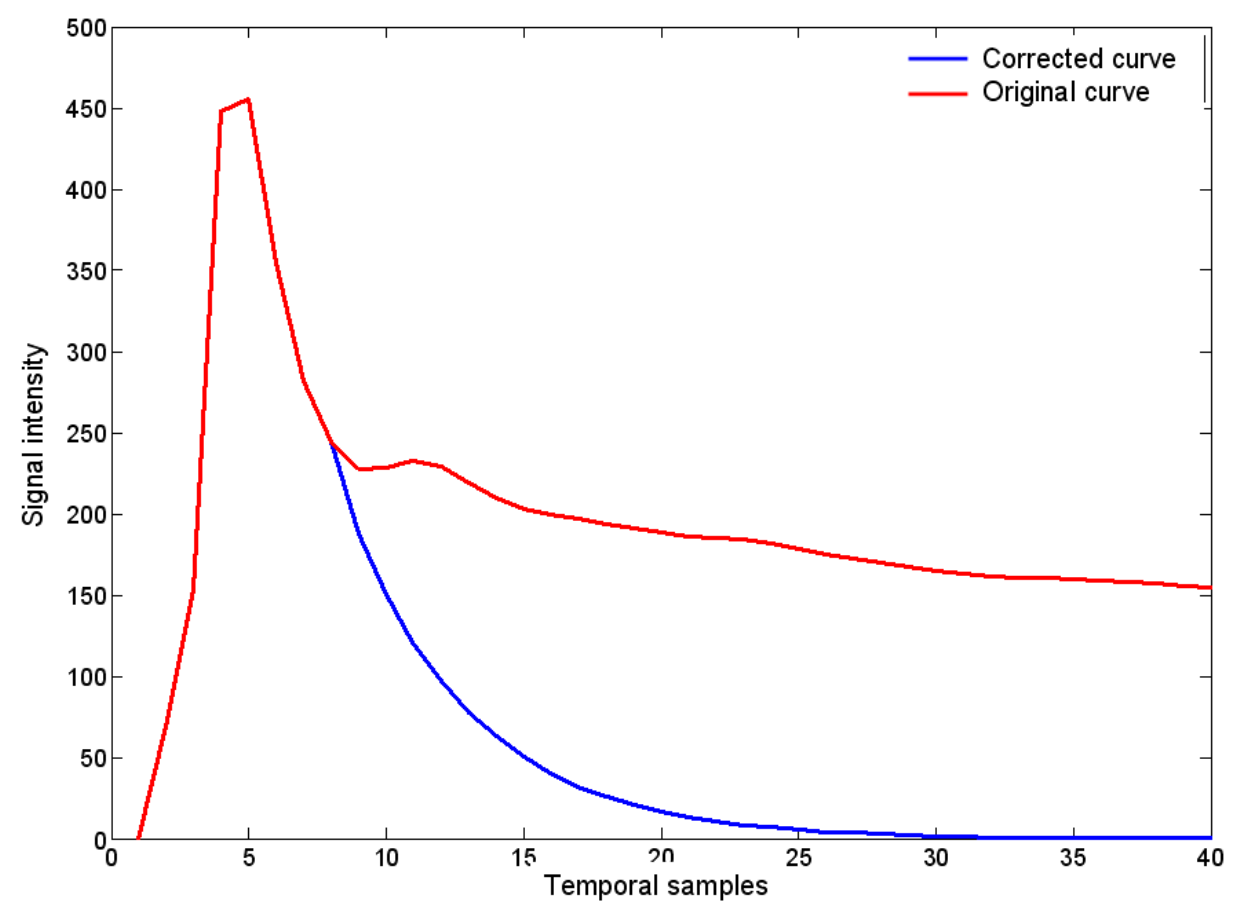

Figure 2.13. Correction of the recirculation and the washout phases of a voxel enhancement curve. The corrected curve decay is fitted to an exponential function.

Then the following parameters were obtained:

- Cerebral blood volume $(C B V)$ : obtained as the area under the contrast uptake curve normalized to the area under the AIF.

$$
C B V=\frac{\int_{0}^{t} C_{\text {corr }} d t}{\int_{0}^{t} C_{c o r r}^{A I F} d t}
$$

- Mean transit time (MTT): obtained as the area under the contrast uptake curve normalized to its maximum.

$$
M T T=\frac{\int_{0}^{t} C_{c o r r} d t}{C_{c o r r}^{\max }}
$$


- Cerebral blood flow $(C B F)$ : obtained as the relationship between the $C B V$ and the MTT.

$$
C B F=\frac{C B V}{M T T}
$$

Multiple-compartment model (two-compartment model)

As previously explained in the Introduction, this model is based on the exchange of contrast agent between plasma and interstitial space.

$$
C=v_{p} \cdot C+K^{\text {trans }} \int_{0}^{t} C_{A I F} \cdot e^{-k_{e p}\left(t-t^{\prime}\right)} d t^{\prime}
$$

In comparison to the single-compartment approach, no recirculation correction of the curves was taken into account to apply this model.

First-pass multiple-compartment model

As a variation of the previous two-compartment model, the following expression can be used to assess only the first-pass phase of the tissue uptake [19]:

$$
C=v_{p f p} \cdot C_{A I F}+K^{f p} \int_{0}^{t} C_{A I F} d t^{\prime}
$$

where $v_{p f p}$ and $K f p$ are the first-pass vascular space fraction and capillary permeability, respectively. It can be seen that with this approach the tissue response is not taken into account. 


\section{$\underline{\text { Histogram analysis }}$}

Mean, median, mode and standard deviation were calculated for the whole histogram and for the 10\% maximum values (figure 2.14). Kurtosis and skewness of the whole histogram were also calculated as shape descriptors. With this analysis it was intended to characterize the regions according to the maximum values, related to tumor aggressiveness [111], and according to the histogram shape, related to the heterogeneity.

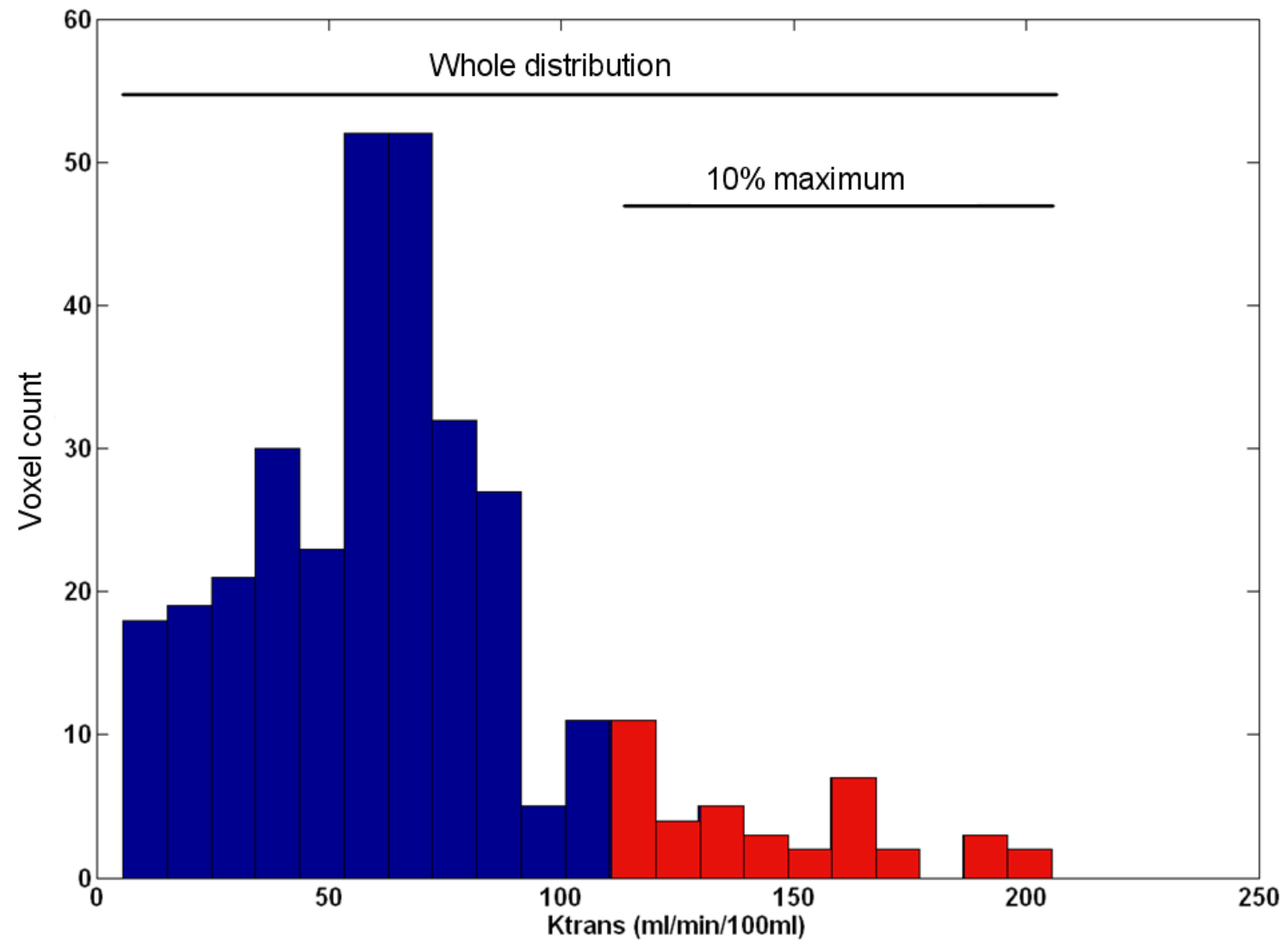

Figure 2.14. Example of a histogram where two groups of voxels are analyzed separately: the whole distribution and the subgroup containing the $10 \%$ maximum values.

\section{$\underline{\text { Statistical analysis }}$}

An ANOVA with post-hoc tests was used to study differences between the three regions for each parameter. Also, in an aim to obtain more statistical power to separate regions, a discriminant analysis was performed. From this analysis a 
statistical function can be generated as a linear combination of several perfusion parameters, which maximizes the capacity to separate the regions.

\subsubsection{Magnetic field strength influence}

\section{Description}

To study the value ranges and the reproducibility of the PK parameters derived from DCE-MR images of the patellar cartilage in $1.5 \mathrm{~T}$ and $3.0 \mathrm{~T}$.

\section{Hypothesis}

As PK modeling is a theoretically reproducible technique, there are no differences in the quantitative results regarding magnetic field strength.

\section{Study group}

16 subjects who underwent a DCE-MR examination of the knee were retrospectively included in the study. All of them were healthy subjects without degenerative patellar conditions. Subjects were studied in $1.5 \mathrm{~T}$ and $3.0 \mathrm{~T}$ magnets. Both groups were all male and similar in age $(26.7 \pm 2.7$ and $24.5 \pm 4.8$ years old). Also, a prospective evaluation was designed examining 4 healthy subjects on both MR fields within 1 week.

\section{Image acquisition}

The acquisition parameters used for each MR examination are indicated in table 2.6 .

\section{$\underline{\text { Image analysis }}$}

The same image analysis process as in the previous study cases of the cartilage was carried on (2.2.1.4). 


\begin{tabular}{ccc}
\hline & PK study 1 & PK study $\mathbf{2}$ \\
\hline Magnetic field & $1.5 \mathrm{~T}$ & $3.0 \mathrm{~T}$ \\
Sequence & T1w sGRE & T1w sGRE \\
Orientation & Sagittal & Sagittal \\
Fat suppression & Yes & Yes \\
Repetition time & $4.0 \mathrm{~ms}$ & $3.48 \mathrm{~ms}$ \\
Echo time & $1.72 \mathrm{~ms}$ & $1.86 \mathrm{~ms}$ \\
Flip angle & $10^{\circ}$ & $10^{\circ}$ \\
Slices & 10 & 10 \\
Voxel size & $0.78 \times 0.78 \times 6 \mathrm{~mm}$ & $0.76 \times 0.76 \times 6 \mathrm{~mm}$ \\
Dynamics & 50 & 50 \\
Temporal resolution & $2.9 \mathrm{~s}$ & $2.9 \mathrm{~s}$ \\
Acquisition time & $2 \mathrm{~min} 25 \mathrm{~s}$ & $2 \mathrm{~min} 25 \mathrm{~s}$ \\
Contrast dose & $0.2 \mathrm{ml} / \mathrm{kg} @ 4 \mathrm{ml} / \mathrm{s}$ & $0.2 \mathrm{ml} / \mathrm{kg} @ 4 \mathrm{ml} / \mathrm{s}$ \\
& $($ Dotarem, Guerbet $)$ & $($ Dotarem, Guerbet)
\end{tabular}

\section{Intensity to concentration conversion}

To convert the intensity vs. time curves into concentration vs. time curves, it was assumed that the normalized intensity vs. time curve was linearly related to the contrast agent concentration [17], as explained in previous study cases of the cartilage.

\section{PK modeling}

In this study a one-input and two-compartment model was used (the expression for this model can be found in the previous applications, 2.2.1.3 and 2.2.1.4).

\section{$\underline{\text { Statistical analysis }}$}

A Student's t-test for independent samples was used for comparisons. A pvalue $<0.05$ was considered as statistically significant. For the test-retest analysis of variability the RMS_CoV was calculated. 


\subsubsection{Curve fitting}

For all the study cases presented in this thesis, a non-linear least squares curve fitting algorithm (Levenberg-Marquardt) was used [83]. It is not the aim of this work to provide a deep analysis of curve fitting algorithms, so in this section only this algorithm will be briefly described.

In least square fitting algorithms, the best fit between the model and the observed data is that instance of the model for which the sum of squared residuals (difference between the value of the model and the value of the observed data) has its least value.

The Levenberg-Marquardt algorithm can be summarized as the minimization of:

$$
S(\beta)=\sum_{i=1}^{m}\left[y_{i}-f\left(x_{i}, \beta\right)\right]^{2}
$$

where $y_{i}$ is the observed data, $x_{i}$ is the independent variable and $\beta$ is the set of parameters to optimize.

It works as an iterative procedure, where the user needs to supply initial values for $\beta$. Depending on the problem to be fitted, one initialization or another may converge to different results, either to a local or a global minimum.

In each iteration, the parameter $\beta$ is replaced by $\beta+\delta$. This variation $\delta$ needs to be estimated. As it is supposed to be small, the function can be written as:

$$
f\left(x_{i}, \beta+\delta\right) \approx f\left(x_{i}, \beta\right)+J_{i} \delta
$$

where

$$
J_{i}=\frac{\partial f\left(x_{i}, \beta\right)}{\partial \beta}
$$


Taking into account that when the minimum of the sum of squares is reached $(S)$, the gradient of $S$ with respect to $\beta$ must be 0 , then the following set of linear equations can be obtained in order to calculate $\delta$.

$$
\left(J^{T} J+\lambda I\right) \delta=J^{T}[y-f(\beta)]
$$

where $J$ is the Jacobian matrix whose $i$-th row equals $J_{i}, \lambda$ is a non-negative damping factor, and $f$ and $y$ are vectors with $i$-th component $\mathrm{f}\left(x_{i}, \beta\right)$ and $y_{i}$, respectively.

\subsubsection{New developments}

\subsubsection{Assessment of the arterial contribution in liver carcinomas}

In the modified Tofts model (or St. Lawrence-Lee adiabatic approach), the vascular contribution to the tissue curve consists of a normalized coefficient which acts as a weighting factor applied to the AIF. However, this approach does not consider the change in shape which occurs when comparing the function sampled at an artery with the function sampled at the tissue. In most cases, when a vascular component can be observed at the tissue enhancement curve, it visually corresponds to dispersion (scale and delay) and even a skewness (shaping) of the AIF bolus.

It has been discussed that one of the most important characteristics of liver tumors is how the vascularization of the lesion changes from a predominant portal blood supply towards a much more arterialized contribution. Therefore it is it necessary to develop imaging tools to assess both the amount of new arterial vessels and the degree of heterogeneity in the lesions, not only to study pathology in a quantitative and reproducible way, but also to evaluate the efficacy and effectiveness of antiangiogenic treatments [2]. A new approach 
based on the application of derivatives and gamma probability density functions is proposed to separate vascular and washout phases in order to assess arterial vascular contribution more accurately, taking into account both the scale and the shape of liver enhancement curves. A new arterial index (AI) is calculated which allows to quantify the degree of similarity between any enhancement curve and a reference AIF, such as the aorta. This AI is compared with the vascular space fraction and the permeability component as calculated from the generalized pharmacokinetic model. A voxel-based approach is used in order to obtain the derived parametric maps to study both liver and tumor regions separately.

\section{Separation of first pass and washout for the AIF}

The characteristic AIF has two main phases: first pass (fast initial rise and fall) and washout (slow decay). This separation can be done in a reproducible way by calculating the curve gradients. When the value of the gradient is high, it means that the curve changes rapidly (initial peak rise and initial peak descent, backflow peak rise and backflow peak descent) and when it is low, it means the curve has reached more steady phases (washout phase). With this procedure, the first pass phase of the AIF can be extracted (figure 2.15). This new curve can be modeled using a gamma probability density function:

$$
\begin{gathered}
C_{a}(t)=C_{a}^{F P}(t)+C_{a}^{W O}(t) \\
C_{a}^{F P}(t)=t^{\alpha-1} \frac{e^{-t / \beta}}{\Gamma(t) \cdot \beta^{\alpha}}
\end{gathered}
$$

where $C_{a}$ is the AIF, $C_{a}{ }^{F P}$ and $C_{a}$ WO are the first pass and the washout phase of the AIF, respectively, $\alpha$ is a shape factor, $\beta$ is a scale factor and $\Gamma(t)$ is the value of the gamma function at time $t$. 


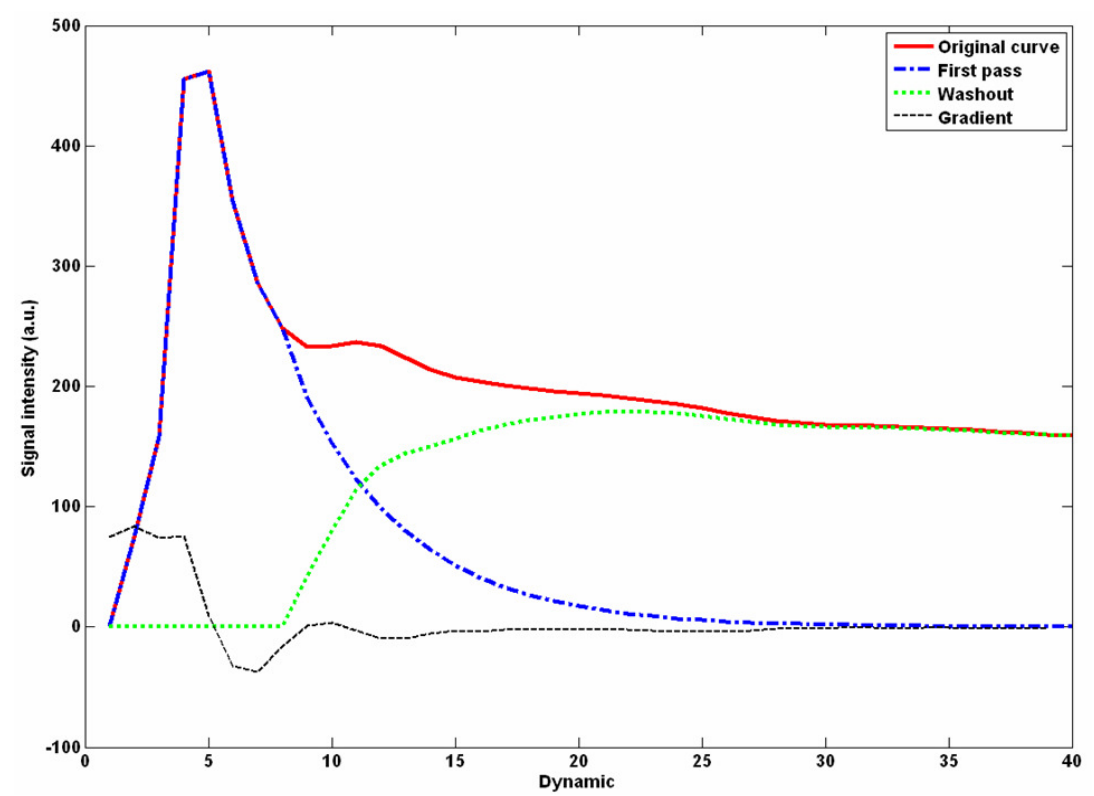

Figure 2.15. Separation of the AIF in 2 phases: first pass and washout, from the information given by the gradients. First pass downslope is generated as a smooth exponential decay.

The combination of factors $\alpha$ and $\beta$ in the gamma function produces different shapes which can be used to model the behavior of the arterial bolus dispersion and change of shape (figure 2.16). For simplicity, recirculations were included as part of the washouts.

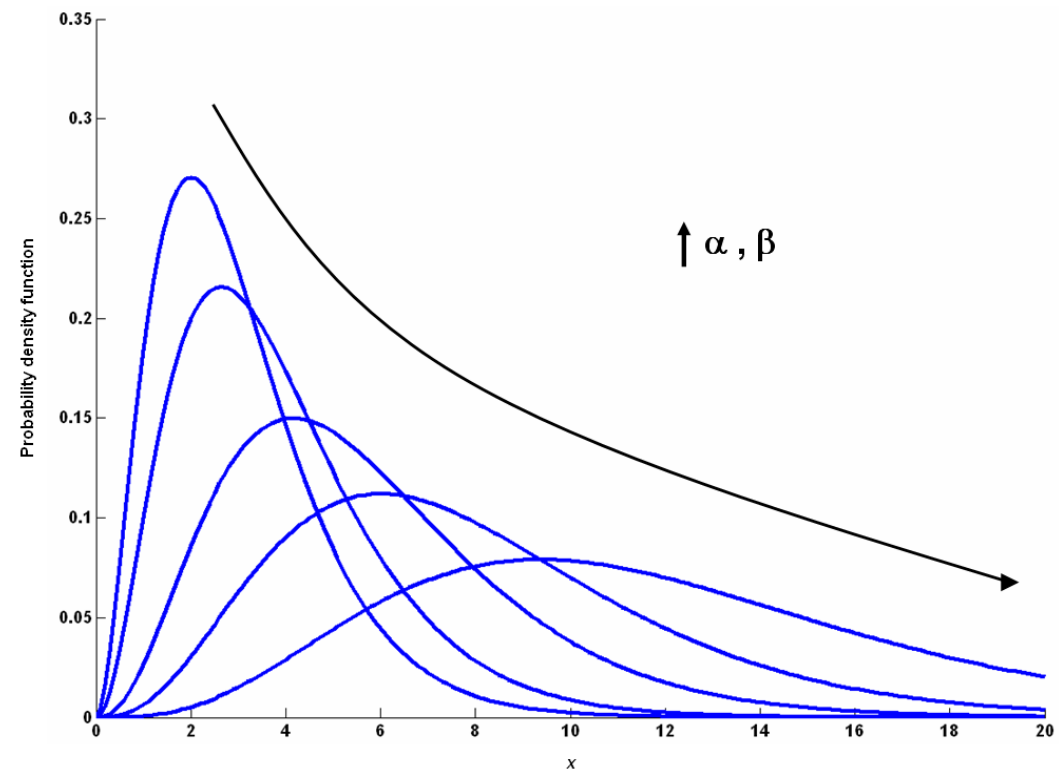

Figure 2.16. Gamma probability density functions. It can be seen that a progressive decay and dispersion is produced as the combination of $\alpha$ and $\beta$ increases. $x$ represents a random variable. 
$\underline{\text { Separation of first pass and washout for the tissue enhancement curves }}$

Enhancement curves were considered as arterially significant if they complied with two criteria: a) their maximum value was higher than the mean plus 3 standard deviations of the values corresponding to the second half of the curve (i.e., the washout phase), and b) their maximum occurred before the maximum portal input. If these conditions were met, the curves were then separated using the gradients algorithm. Otherwise the curves were considered as only washout (figure 2.17).

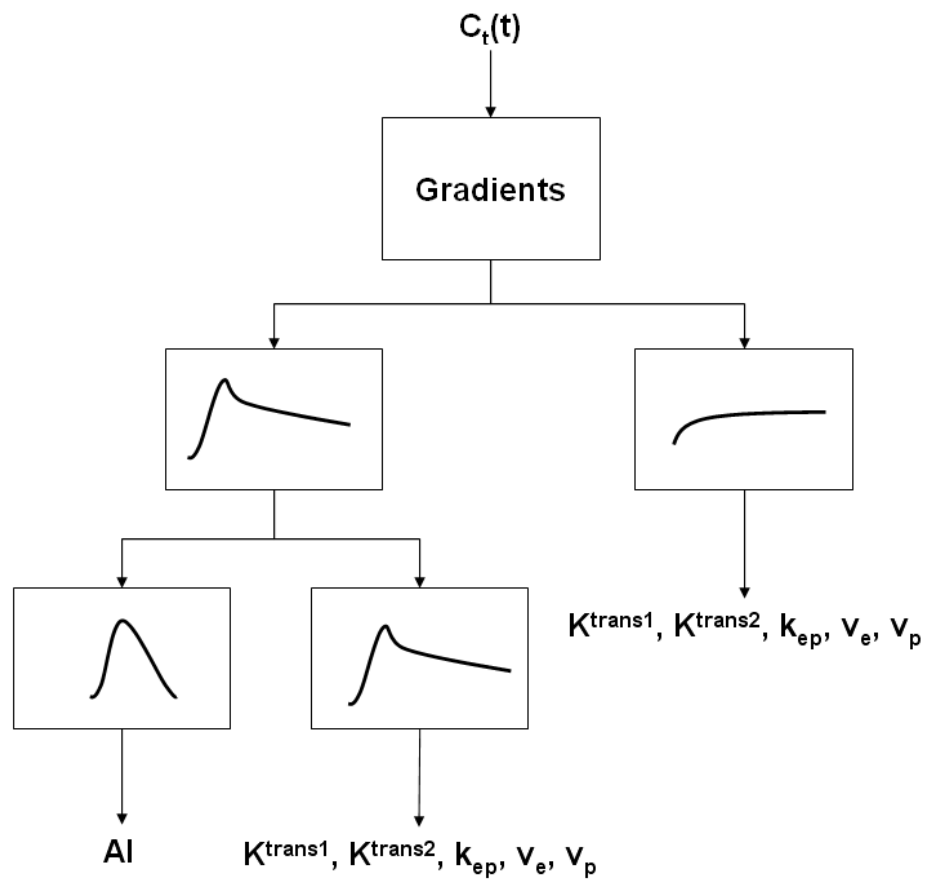

Figure 2.17. Proposed algorithm for the extraction of the pharmacokinetic parameters by separation of enhancement curves into arterially and non-arterially significant. When arterially significant, curves are separated into first pass and washout using the gradient, and the $A I$ and the common pharmacokinetic parameters $\left(K^{\text {trans } 1}, K^{\text {trans } 2}, k_{e p}, v_{e}, v_{p}\right)$ are calculated separately.

\section{$\underline{\text { PK modeling }}$}

The two-input two-compartment generalized kinetic model [69] adapted to the liver dual input is used:

$$
C_{t}(t)=v_{p} C_{a}(t)+\int_{0}^{t}\left(K^{\text {trans } 1} C_{a}(\tau)+K^{\text {trans } 2} C_{v}(\tau)\right) e^{-k_{e p}(t-\tau)} d \tau
$$


where $C_{t}$ is the tissue enhancement curve, $C_{v}$ is the portal input function, $K^{\text {trans } 1}$ is the capillary permeability for the arterial contribution, $K^{\text {trans } 2}$ is the capillary permeability for the venous contribution, $v_{p}$ is the vascular space fraction and $k_{e p}$ is the washout coefficient. The interstitial space fraction, $v_{e}$, is obtained as $\left(K^{\text {trans1 }}+\right.$ $\left.K^{\text {trans } 2}\right) / k_{e p}$ [7]. Pixels whose values were outside the range $[0,1]$ for this parameter were automatically discarded from the analysis. All curves were fitted using Levenberg-Marquardt non-linear least squares algorithm [83].

The arterial index $(A I)$ can be calculated using the following expression:

$$
A I=\frac{\alpha_{a}+\beta_{a}}{\alpha_{t}+\beta_{t}}
$$

where $\alpha_{t}$ and $\beta_{t}$ are the $\alpha$ and $\beta$ factors for the tissue first-pass curve, and $\alpha_{a}$ and $\beta_{a}$ are the $\alpha$ and $\beta$ factors for the arterial input first-pass curve. Values for this index range from 0 to 1 . Near 1 values of this index correspond to highly arterialized curves while near 0 values correspond to minimally or no arterialized curves (i.e. curves with more dispersion). This index provides the degree of similarity between any arterialized tissue enhancement curve and the aortic enhancement curve.

Colored parametric images were generated for each variable $\left(A I, K^{\text {trans } 1}, K^{\text {trans } 2}, k_{e p}\right.$, $v_{e}$ and $v_{p}$ ) to assess regional differences.

\section{Study group and image acquisition}

Fifteen cirrhotic patients with hepatocellular carcinoma (8 men, 7 women, $63 \pm 13$ years), not treated previously and with a clinical and analytical follow-up of 9 months were included.

All patients were imaged in a $1.5 \mathrm{~T}$ scanner (Philips Intera, Philips Healthcare, Best, The Netherlands). A 4-channel phased array surface coil was used. To perform the kinetic analysis, the following sequences were acquired: 


\begin{tabular}{ccc}
\hline & T1 mapping & PK study \\
\hline Magnetic field & $1.5 \mathrm{~T}$ & $1.5 \mathrm{~T}$ \\
Sequence & T1w sEG & T1w sEG \\
Orientation & Transversal & Transversal \\
Fat suppression & No & Yes \\
Repetition time & $87 \mathrm{~ms}$ & $70 \mathrm{~ms}$ \\
Echo time & $1.4 \mathrm{~ms}$ & $1 \mathrm{~ms}$ \\
Flip angle & $5^{\circ}, 15^{\circ}, 30^{\circ}, 45^{\circ}, 70^{\circ}$ & $60^{\circ}$ \\
Slices & 24 & 24 \\
Voxel size & $0.8 \times 0.8 \times 7.5 \mathrm{~mm}$ & $0.8 \times 0.8 \times 7.5 \mathrm{~mm}$ \\
Dynamics & - & 40 \\
Temporal & - & $3.7 \mathrm{~s}$ \\
resolution & $2 \mathrm{~min}$ & $2 \mathrm{~min} 28 \mathrm{~s}$ \\
Acquisition time & - & $($ Dotarem $)$ \\
Contrast dose & & $0.05 \mathrm{ml} / \mathrm{kg} @ 4 \mathrm{ml} / \mathrm{s}$ \\
\hline Table 2.3. Sequence parameters for the liver assessment in $1.5 \mathrm{~T}$. \\
\hline
\end{tabular}

The patients were asked to breathe slowly during the dynamic study. No special consent from the ethics committee was required, as this study is done routinely in suspected liver tumors. After acquisition, all images were anonymized and transferred to a workstation for post-processing.

\section{$\underline{\text { Image analysis }}$}

All images were registered to correct for breathing motion misalignments with the SPM non linear registration tool (SPM, Wellcome Trust Center for Neuroimaging, London, UK).

Multiple flip angle images were used to calculate pre-contrast T1 maps of the liver. Signal intensity vs. flip angle curves were extracted for each pixel and fitted to the 3D spoiled gradient echo equation [112]. 
To perform the kinetic analysis the arterial input, the portal input and the tissue enhancement curves were initially obtained. To generate the vascular input functions, two regions of interest (ROIs) were manually placed at the aorta and at the portal vein and all the pixels within the ROIs were averaged for each time step. The liver was also manually segmented and a tissue enhancement curve was extracted for each pixel to be independently analyzed (figure 2.18).

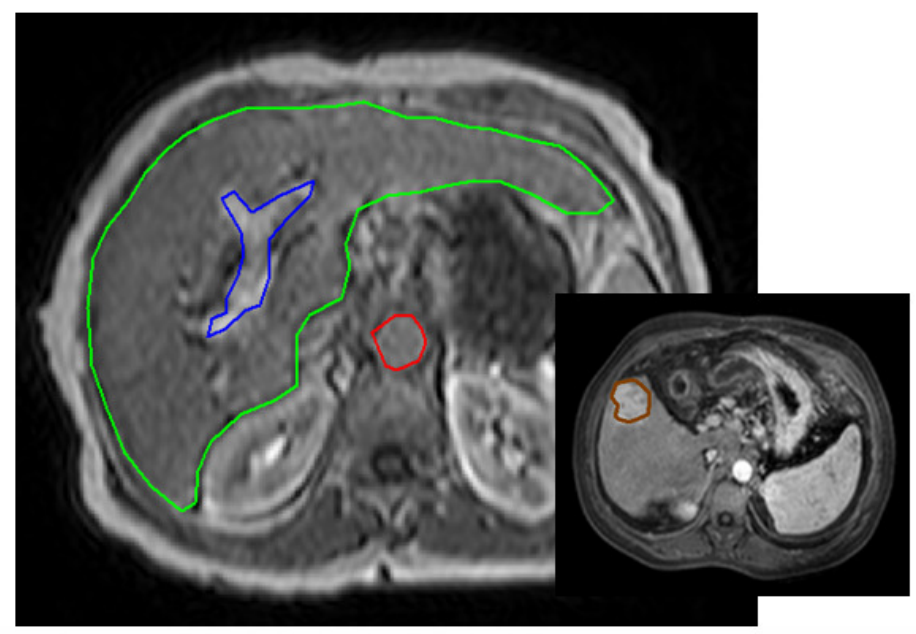

a

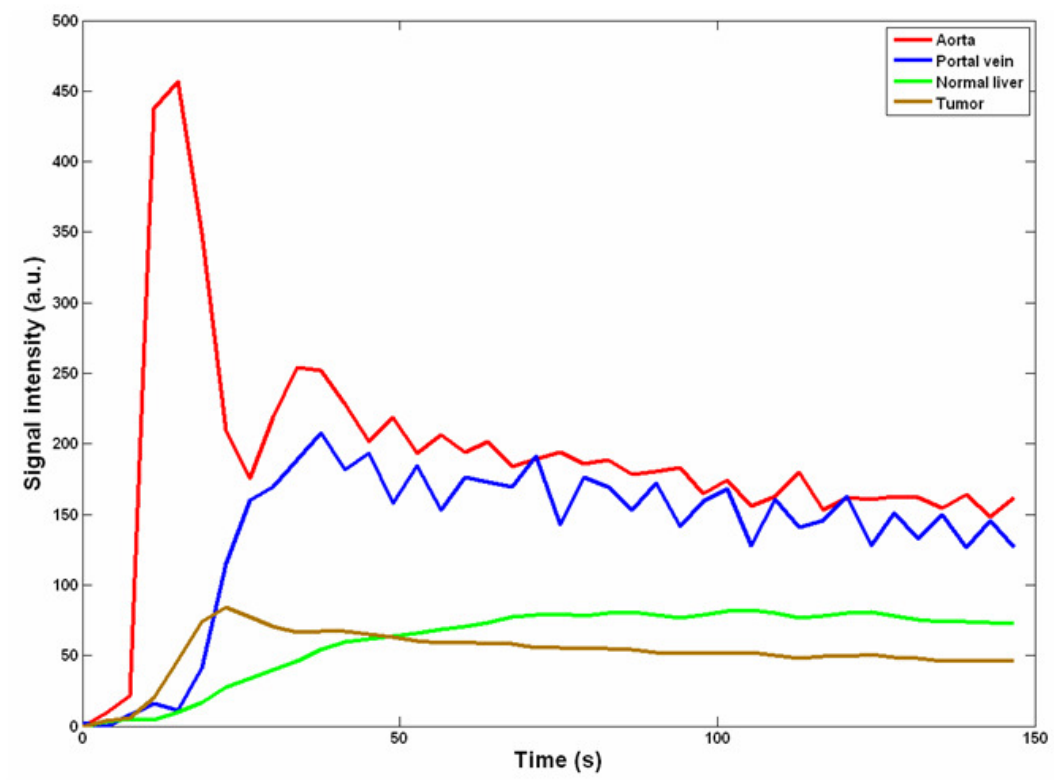

b

Figure 2.18. (a) Regions of interest for the PK analysis of the liver: aorta (red), portal vein (blue), tumor (brown) and liver parenchyma (green). (b) Typical normalized enhancement functions at the aorta, the portal vein, the normal liver and the carcinoma. 
All curves were smoothed to reduce the noise using local regression with weighted linear least squares and first degree polynomials. They were then normalized and converted into concentration values.

\section{$\underline{\text { Statistical analysis }}$}

The values for each calculated parameter were expressed as the mean $\pm \mathrm{SD}$ and the median for the liver and the tumor. Linear dependence between $\mathrm{K}^{\text {trans1 }}$ and $\mathrm{AI}$, and between $\mathrm{v}_{\mathrm{p}}$ and $\mathrm{AI}$ was assessed using the Pearson's correlation coefficient ( $r$ ) for the liver and the tumor.

\subsubsection{Cartilage parametric maps}

The study of the cartilage by clinical arthroscopy reveals that the cartilage may suffer regional degenerations and pathological processes. Therefore, characterizing it as a whole using the mean or the median as quantitative indicators, may surely be leading to important errors, especially if the affected areas are small.

A new visualization tool is proposed to assess regional cartilage properties. It consists in the generation of synthetic colored maps for each parameter using all the cartilage voxel.

Knee or ankle images are usually acquired in the sagittal plane to cover all the relevant structures. After the cartilage (patellar, femoral, tibial, astragalus) has been properly segmented and all the voxel curves have been studied to obtain the PK parameters, a series of projections can be made in order to generate multiplanar colored maps. Depending on the cartilage under study, one projection direction or another is chosen.

For each projection, each line corresponds to a pixel in the parametric map. All the voxels that the line runs through are averaged to calculate the value of the pixel. 
- Patellar cartilage: left-right projection (figure 2.19).
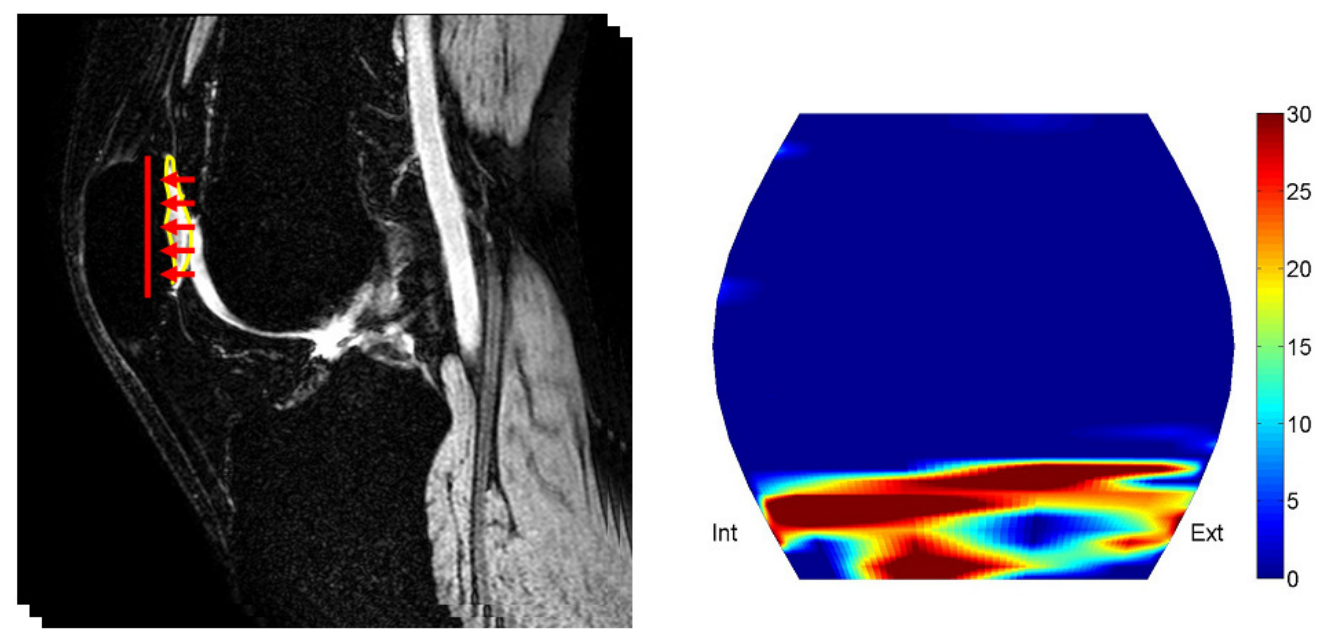

Figure 2.19. $\mathrm{K}^{\text {trans }}$ coronal projection of the sagittal slices for the patellar cartilage. The red arrows represent the direction in which the values are averaged to generate the final projection.

- Femoral cartilage: radial projection (figure 2.20).
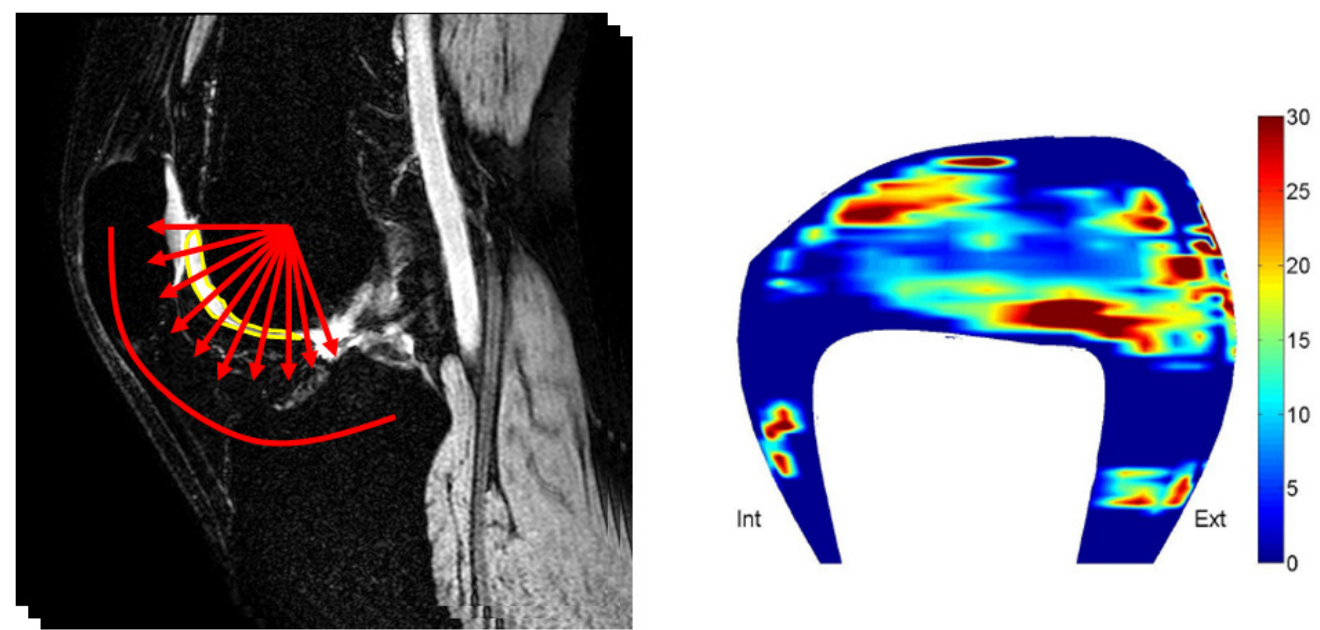

Figure 2.20. $\mathrm{K}^{\text {trans }}$ radial projection of the sagittal slices for the femoral cartilage. The center must be specified manually. 
- Tibial cartilage: bottom-up projection (figure 2.21).
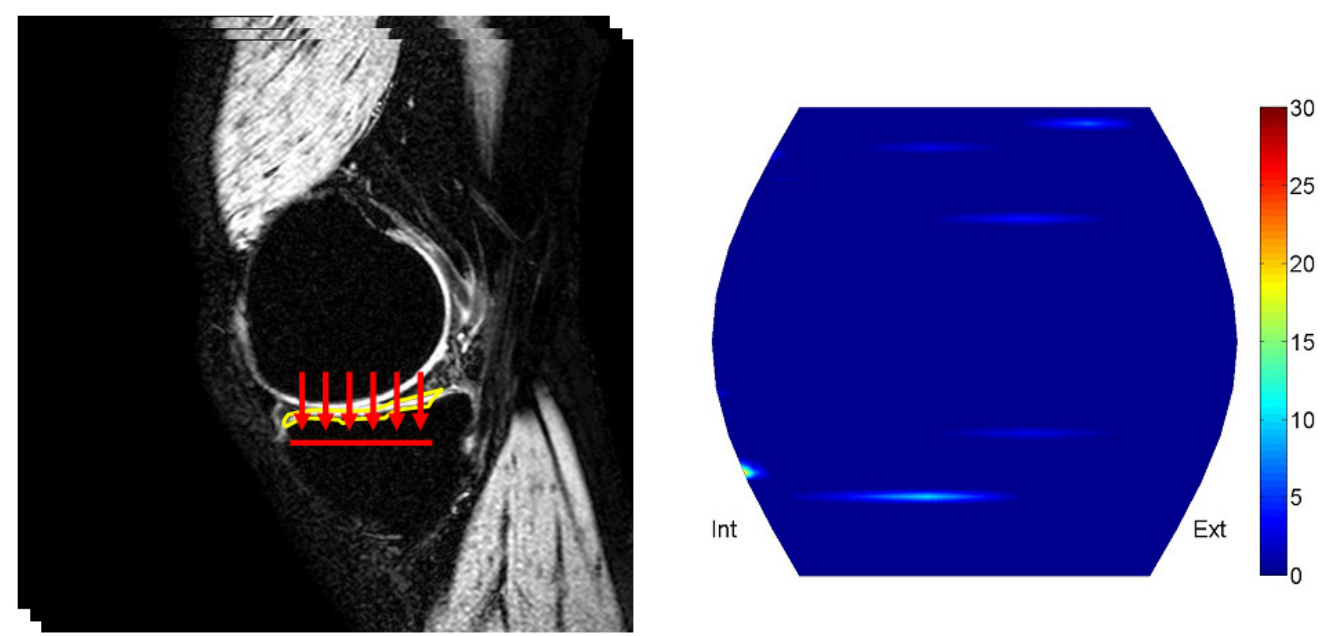

Figure 2.21. $\mathrm{K}^{\text {trans }}$ axial projection of the sagittal slices for the tibial cartilage.

- Ankle cartilage (tibio-astragalin): bottom-up projection (figure 2.22).
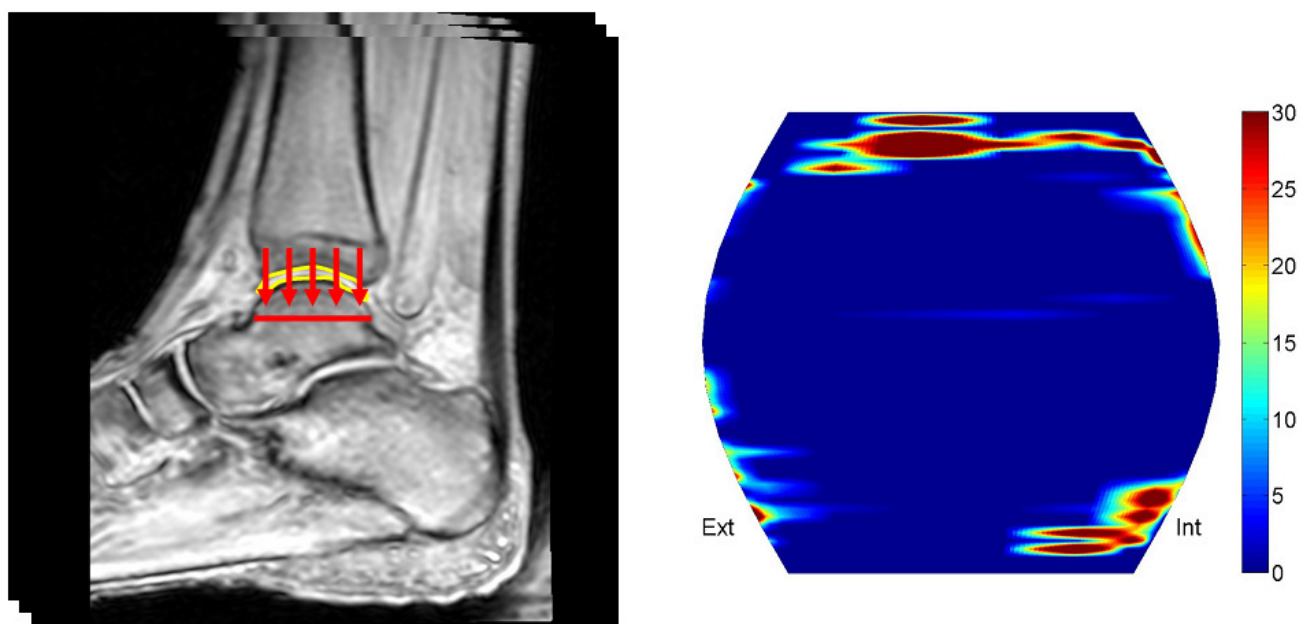

Figure 2.22. Ktrans axial projection of the sagittal slices for the ankle cartilage. 
It can be seen that all these images can be used to assess regional damage. However, this simple methodology has some limitations regarding projection errors.

As absolute coronal or transversal projections are chosen, it is possible that the cartilage may not be correctly aligned with these planes, so that it is not completely perpendicular. Moreover, cartilages have no plane volumes so depending on the region of the cartilage there may be more voxels being averaged at a time. These factors will lead to parametric images which will not reflect the cartilage as expected and, more importantly, make it very difficult to compare one cartilage through consecutive studies, as the knee position will not probably be the same.

One way to solve this bias is by using a segmentation of the bone (which is not expected to change significantly between studies) to that the cartilage is projected not on an absolute plane, but on the bone surface [113]. This approach does not produce $2 \mathrm{D}$ parametric images, but rather $3 \mathrm{D}$ reconstructions of these parametric images. This later approach is currently being implemented.

\subsubsection{Multivariate image analysis (MIA)}

The study of a disease by analyzing several parameters allows having a wide amount of information to characterize it in great detail. However, these parameters are often related among them and it is sometimes difficult to avoid redundancies and extract accurate knowledge. There are data mining tools based on the multivariate and the factorial analysis of the information which can be used to reduce the dimensionality and help establish classifications from the combination of several parameters into discriminant functions [114,115]. These classifiers can then be used to group patients (clusters) with similar characteristics, which would be otherwise impossible to do with univariate 
studies. The multivariate analysis of perfusion imaging biomarkers may provide new ways to assess the diseases.

Two tools are proposed to improve diagnose and prognosis with perfusion imaging biomarkers:

\section{Principal component analysis (PCA)}

PCA is the workhorse of MIA. The key is the proper reorganization (matricizing) of the original $\mathrm{N}$-way array. Unfolding is done so that each voxel becomes a single row in the analysis. Thus an image that is originally $I \times J$ with $K$ spectral channels is reshaped to form a two way array that is $I \times J \times K$ [116].

PCA consists of an orthogonal linear transformation that converts the original data into a new coordinate system so that the greatest variance by any projection of the data lies on the first coordinate (first principal component), the second greatest variance on the second coordinate (second principal component), and so on. It involves the computation of the $K$ images covariance matrix and the application of diagonalization procedures for extracting the eigenvalues and the corresponding eigenvectors. A score plot can then be obtained so that the original data is projected onto the principal components in two dimensions. The score plot is a scatter plot, where the $\mathrm{X}$ axis contains a one principal component and the $\mathrm{Y}$ axis contains another principal component. This plot contains points that represent the original parameters (variables) projected onto the selected principal components. The score plot is usually built with the data on the first two principal components.

\section{Linear discriminant analysis (LDA)}

Linear discriminant analysis (LDA) and its related Fisher's linear discriminant analysis are methods used to find the linear combination of variables which best separate two or more classes. The resulting combination may be used as a linear classifier or for dimensionality reduction before later classification. 
LDA is also closely related to PCA and factor analysis in that both look for linear combinations of variables which best explain the data [117]. The aim of LDA is to obtain a function which can be further used in order to generate colored nosologic images which are directly linked to the disease. So, instead of providing information on quantitative parameters (such as permeability, flow, etc.), they will provide a direct index of disease.

Figure 2.23 shows some examples of parametric maps of the ankle cartilage and related nosologic images.

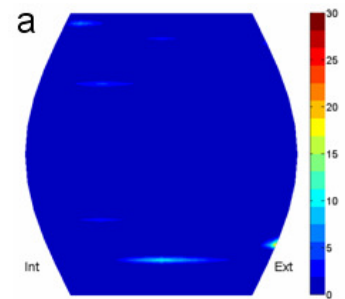

$K^{\text {trans }}$

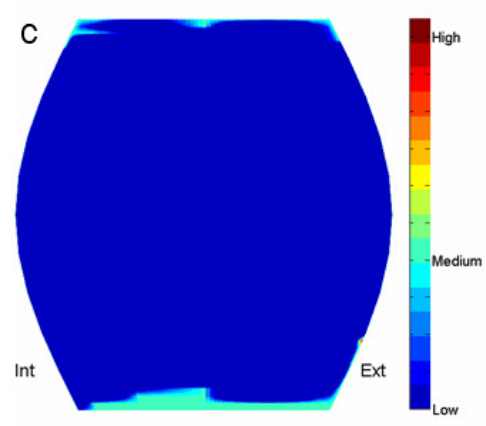

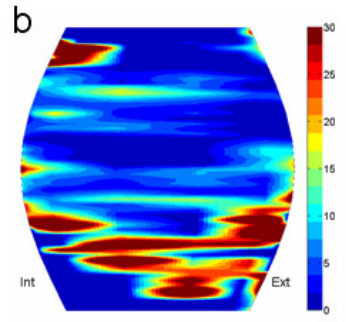

$K^{\text {trans }}$

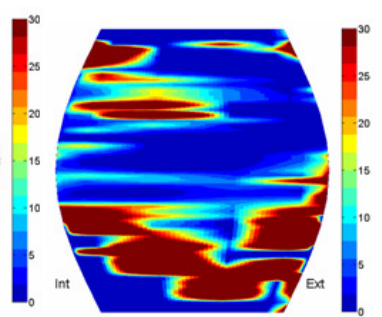

$\mathbf{v}_{\mathrm{e}}$

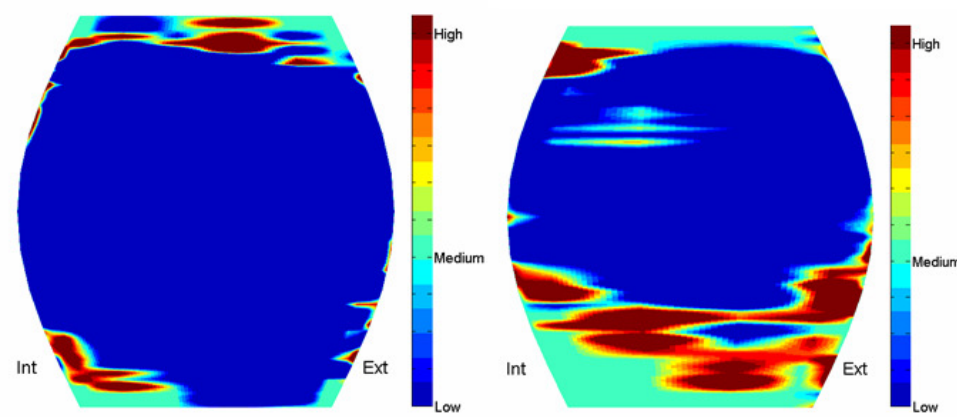

Figure 2.23. Parametric maps of the ankle cartilage for (a) a normal subject and (b) a patient with osteoarthritis. (c) Nosologic maps obtained from a discriminant function in three cases (from left to right: normal, initial degeneration and advanced degeneration).

\subsubsection{Assessment of region heterogeneities}

As it has been already introduced, tumor regions have chaotic vascular structures, with arterio-venous shunts and fragile vessels. This type of composition makes tumors highly heterogeneous masses, a fact which seems to be directly related to their aggressiveness. 
When tumors are studied with the common statistics, such as the mean, the median, the mode or the standard deviation, it is possible to characterize them relatively well, as the mean, the median and the mode give an idea of the central value and the standard deviation gives an idea of the dispersion of values. However, by using whole region histograms, it is possible to obtain two more statistics which can assess heterogeneity more directly:

\section{$\underline{\text { Kurtosis }}$}

The kurtosis is a measure of the "peakedness" of the histogram. Higher kurtosis means more of the variance is due to infrequent extreme deviations, as opposed to frequent modestly sized deviations. In our context, higher kurtosis is related to less heterogeneous regions.

It is also known as the fourth standardize moment of a distribution:

$$
K=\frac{\mu_{4}}{\sigma^{4}}-3
$$

where $\mu_{4}$ is the fourth moment about the mean (or fourth central moment), which can be defined as:

$$
\mu_{4}=E\left[(X-E[X])^{4}\right]=\int_{-\infty}^{+\infty}(x-\mu)^{4} f(x) d x
$$

where $E$ is the expectation operator (the integral of the variable with respect to its probability measure).

\section{Skewness}

The skewness is a measure of the asymmetry of a histogram. It can be used to assess the shape of a histogram with regard to the apparition of tails over the maximum values. 
It can be defined as:

$$
S=\frac{\mu_{3}}{\sigma^{3}}
$$

where $\mu_{3}$ is the third moment about the mean.

\section{Study case: liver}

Ten patients with hepatocarcinomas were studied using a 2-input bicompartimental PK model in $1.5 \mathrm{~T}$ multislice DCE-MRI of the liver (24 slices, 40 dynamics, $3.6 \mathrm{~s}$ /dynamic). Two regions of interest within the liver parenchyma were manually selected in each case (one for lesion and another for normal parenchyma). Histograms of the five PK parameters were calculated: $K^{\text {trans } 1 \text {, }}$ $K^{\text {trans } 2,} k_{e p}, v_{e}$ and $v_{p}$. Histogram heterogeneity was characterized using the kurtosis . 



\section{Results and discussion}

In this chapter the results corresponding to the clinical applications will be presented: OHSS treatment with cabergoline, cartilage degeneration at $1.5 \mathrm{~T}$ and 3.0 T, osteoarthritic cartilage treatment with glucosamine sulfate, study of gradeIV astrocytomas, influence of magnetic field strength and analysis of hepatocellular carcinomas.

\subsection{Evaluation of ovarian hyperstimulation syndrome treatment}

\subsubsection{Results}

Both PK models (one- and two-compartments) showed a significant increase in $K^{\text {trans }}$ for the second study in the non-treated women group (figure 3.1). However, the differences where bigger in the two-compartment model. The other PK parameters did not show statistically significant differences, although a tendency towards significance was observed for $\mathrm{ve}_{\mathrm{e}}$ in the two-compartment model (table 3.1). In the cabergoline group no significant differences were obtained for any parameter.

For the analysis of percentage differences, statistically significant differences were observed between treatment and placebo for $K^{\text {trans }}$ in the two-compartment model: increments of $168.6 \%$ for the placebo group and of $54.6 \%$ for the treated group $(\mathrm{p}=0.04)$.

For the reproducibility analysis, the ICC showed values of $0.96,0.96$ and 0.98 for $K^{\text {trans }}, k_{e p}$ and $v_{e}$ in the one-compartment model and of $0.97,0.97,0.98$ and 0.89 for $\mathrm{K}^{\text {trans }}, \mathrm{k}_{\mathrm{ep}}, \mathrm{v}_{\mathrm{e}}$ and $\mathrm{v}_{\mathrm{p}}$ in the two-compartment model. 
a

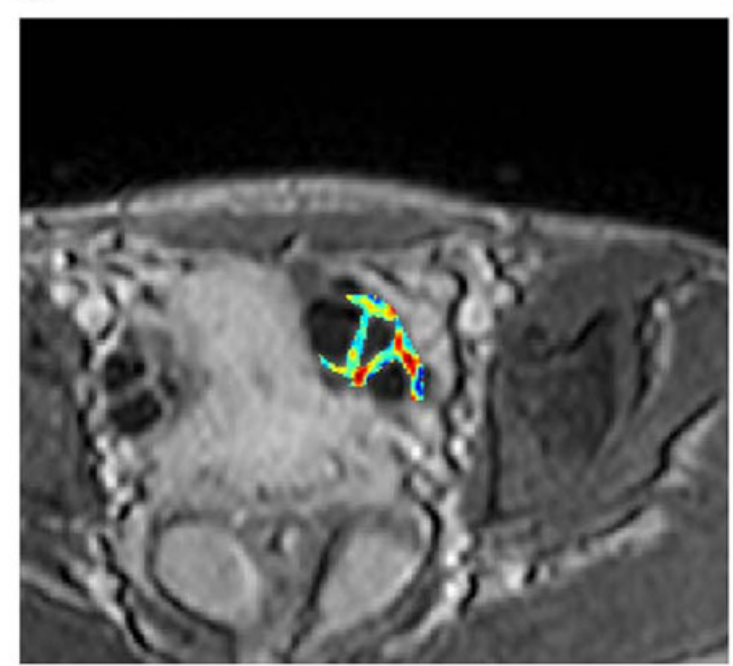

b

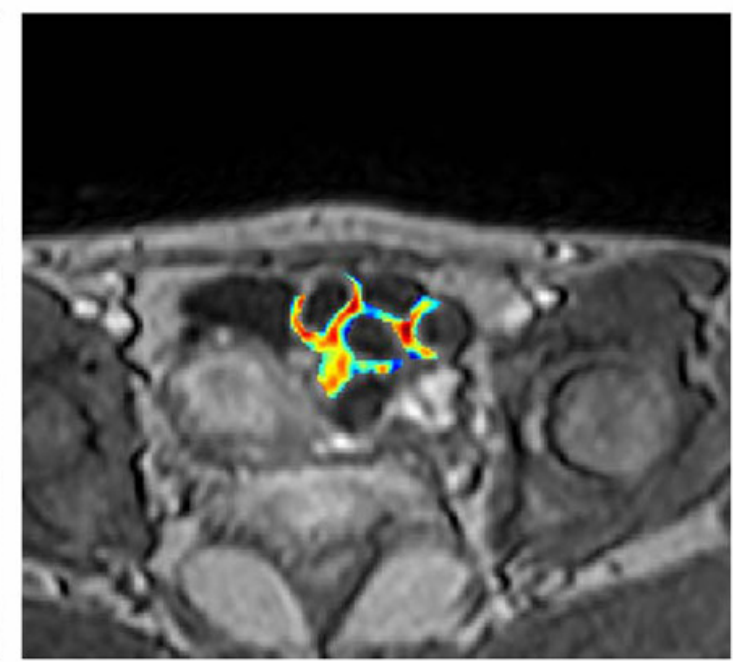

Figure 3.1. $\mathrm{K}^{\text {trans }}$ parametric maps for the first (a) and the second (b) MR studies of a non-treated patient. These parametric maps allow the analysis of regions with higher permeability (red). It can be seen there is an increment of permeability, represented by a higher number of red voxels.

\begin{tabular}{|c|c|c|c|c|c|c|}
\hline Parameter & Group & MR & $\begin{array}{l}\text { 1-compartment } \\
\text { model }\end{array}$ & $p$ & $\begin{array}{c}\text { 2-compartments } \\
\text { model }\end{array}$ & $p$ \\
\hline \multirow{4}{*}{$K^{\text {trans }}$} & \multirow{2}{*}{$\mathrm{T}$} & 1 & $20.4 \pm 6.1$ & \multirow{2}{*}{0.070} & $20.3 \pm 6.7$ & \multirow{2}{*}{0.078} \\
\hline & & 2 & $28.1 \pm 9.1$ & & $28.6 \pm 10.4$ & \\
\hline & \multirow{2}{*}{$\mathbf{P}$} & 1 & $25.1 \pm 10.1$ & \multirow{2}{*}{0.012} & $20.2 \pm 9.6$ & \multirow{2}{*}{$<0.001$} \\
\hline & & 2 & $37.9 \pm 9.1$ & & $42.4 \pm 9.7$ & \\
\hline \multirow{4}{*}{$k_{e p}$} & \multirow{2}{*}{$\mathrm{T}$} & 1 & $80.7 \pm 10.5$ & \multirow{2}{*}{0.115} & $68.7 \pm 12.8$ & \multirow{2}{*}{0.050} \\
\hline & & 2 & $105.2 \pm 40.0$ & & $88.9 \pm 23.4$ & \\
\hline & \multirow{2}{*}{$\mathbf{P}$} & 1 & $101.2 \pm 34.9$ & \multirow{2}{*}{0.345} & $95.4 \pm 55.0$ & \multirow{2}{*}{0.937} \\
\hline & & 2 & $114.4 \pm 21.1$ & & $97.0 \pm 14.6$ & \\
\hline \multirow{4}{*}{$v_{e}$} & \multirow{2}{*}{$\mathrm{T}$} & 1 & $29.2 \pm 5.2$ & \multirow{2}{*}{0.403} & $37.3 \pm 6.2$ & \multirow{2}{*}{0.476} \\
\hline & & 2 & $33.1 \pm 11.8$ & & $41.0 \pm 13.1$ & \\
\hline & \multirow{2}{*}{$\mathbf{P}$} & 1 & $31.3 \pm 8.6$ & \multirow{2}{*}{0.136} & $38.6 \pm 10.6$ & \multirow{2}{*}{0.064} \\
\hline & & 2 & $36.2 \pm 3.2$ & & $46.6 \pm 5.8$ & \\
\hline \multirow{4}{*}{$v_{p}$} & \multirow{2}{*}{$\mathbf{T}$} & 1 & & & $30.3 \pm 12.0$ & \multirow{2}{*}{0.419} \\
\hline & & 2 & & & $22.5 \pm 5.2$ & \\
\hline & \multirow{2}{*}{$\mathbf{P}$} & 1 & - & & $30.3 \pm 12.0$ & \multirow{2}{*}{0.093} \\
\hline & & 2 & & & $22.5 \pm 5.2$ & \\
\hline
\end{tabular}

Table 3.1. Results of the PK parameters for the $1^{\text {st }}$ and $2^{\text {nd }}$ MR studies. $T$ (treated), $\mathrm{P}$ (placebo). Units: $K^{\text {trans }}$ and $k_{e p}(\mathrm{ml} / \mathrm{min} / 100 \mathrm{ml}) ; v_{e}$ and $v_{p}(\%)$ 


\subsubsection{Discussion}

The differences in the PK parameters between the cabergoline treated patients and the non-treated ones demonstrate the effect of the dopamine agonist as OHSS repressor. Although in both groups there was an increase of capillary permeability, this was significantly lower in the treated group in comparison to the non-treated. When analyzing the influence of the PK model, it has been demonstrated that the differences between both groups were higher if the twocompartment model was used.

This study allowed establishing in an objective way the differences between the one- and two-compartment PK models for the characterization of the angiogenesis in the OHSS and the influence of the treatment over these variables. Comparing the PK parameters of capillary permeability, washout rate, interstitial space fraction and vascular space fraction for both models, the significant differences have been validated in a reproducible way between patients treated with cabergoline and patients treated with placebo. In conclusion, these results validate the use of the PK parameters, especially $K^{\text {trans }}$, as imaging biomarkers of the direct effect of antiangiogenic treatments.

In this study the ICC has been used to assess reproducibility. This statistic parameter provides a measure of retest correlation, taking into account the variability between plus within-subject. Another common way of assessing reproducibility is with the test-retest root mean square coefficient of variation (RMSCV), which provides an estimate of the within-subject variation (similar to a normalized standard deviation). The ICC ranges from 0 to 1 , with near 1 values meaning that the method is highly reproducible. On the other hand, RMSCV can take higher values than 1 , but near 0 values are related to high reproducilibity. Despite these differences, both parameters have been used as good reproducibility indicators in this thesis. However, if a thorough study of 
reproducibility is necessary, both parameters should be considered and calculated.

\subsection{Assessment of cartilage degeneration in $1.5 \mathrm{~T}$}

\subsubsection{Results}

In the comparison between the three cartilage groups, the one-compartment model showed significant differences for $K^{\text {trans }}$ and $v_{e}$, with $p$-values of 0.012 and 0.001 , respectively (figure 3.2). $k_{e p}$ was not significantly different $(\mathrm{p}=0.884)$. For the two-compartment model, significant $p$-values were also obtained for $K^{\text {trans }}$ and $v_{e}\left(p=0.02\right.$ and $p=0.007$, respectively), but not for $k_{e p}$ and $v_{p}$ ( $p$-values of 0.897 and 0.379 , respectively) (table 3.2).
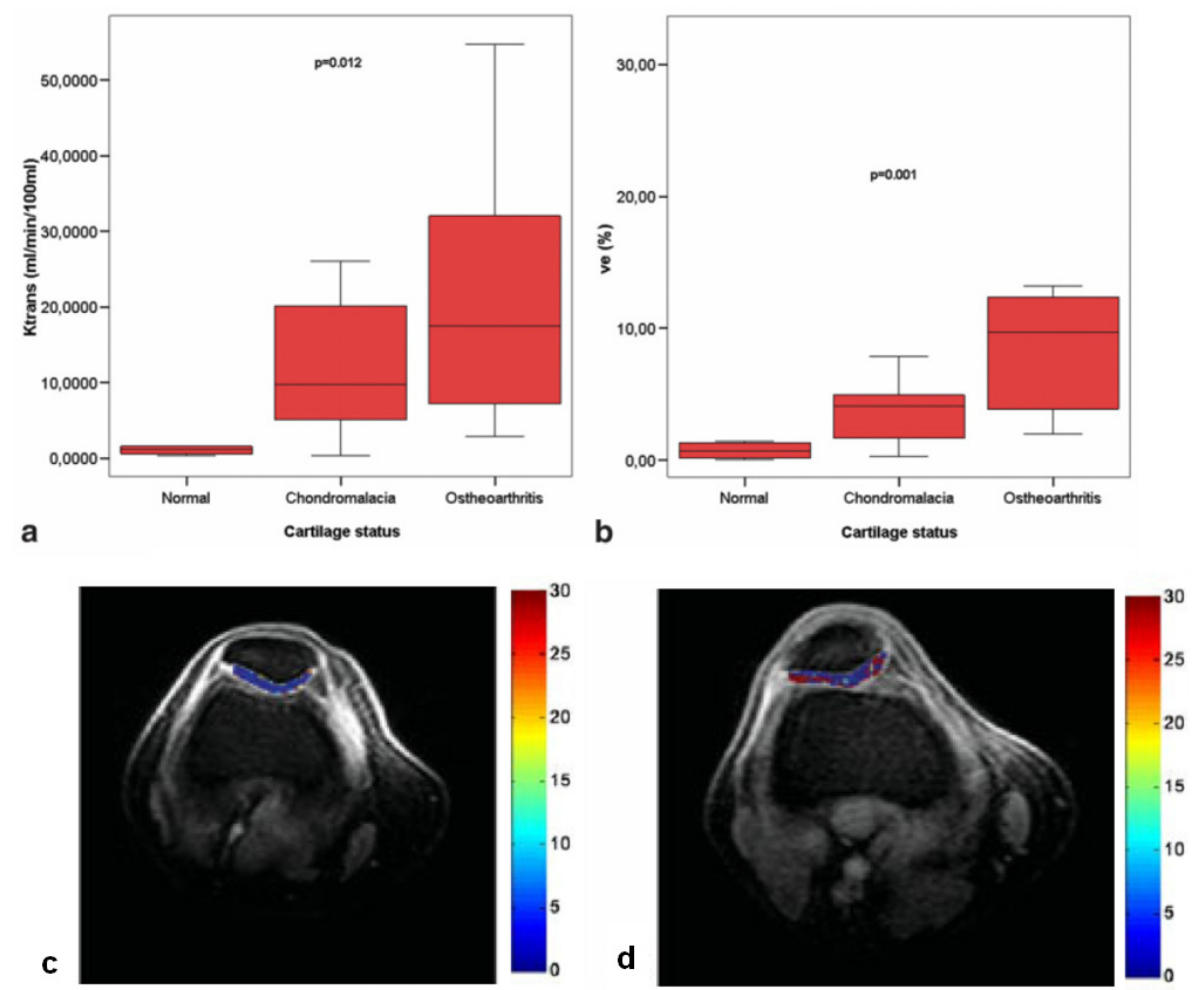

Figure 3.2. Box-plots for (a) $K^{\text {trans }}$ and (b) $v_{e}$. Both parameters are obtained using the Tofts standard method (dismissing vascular contribution). The chart shows the five statistics (minimum, first quartile, median, third quartile and maximum). Cartilage parametric maps for $K^{\text {trans: }}$ (c) shows a normal cartilage; (d) shows a cartilage with osteoarthritis. 


\begin{tabular}{cccccc}
\hline \multirow{2}{*}{ Model } & \multirow{2}{*}{ Parameter } & \multicolumn{3}{c}{ Degeneration status } & \multirow{2}{*}{$\boldsymbol{P}$} \\
\cline { 3 - 5 } & & Normal & Chondromalacia & Osteoarthritis & \\
\multirow{2}{*}{ 1 compart. } & $\boldsymbol{K}^{\text {trans }}$ & $1,1 \pm 0,6$ & $12,0 \pm 8,9$ & $21,2 \pm 16,0$ & 0,012 \\
& $\boldsymbol{k}_{e p}$ & $313,2 \pm 40,4$ & $279,6 \pm 161,1$ & $273,5 \pm 141,0$ & 0,893 \\
& $\boldsymbol{v}_{e}$ & $0,7 \pm 0,7$ & $3,6 \pm 2,2$ & $10,5 \pm 8,2$ & 0,001 \\
& $\boldsymbol{K}^{\text {trans }}$ & $0,2 \pm 0,5$ & $7,7 \pm 6,3$ & $13,2 \pm 10,9$ & 0,021 \\
\multirow{2}{*}{ compart. } & $\boldsymbol{k}_{e p}$ & $352,2 \pm 54,6$ & $311,7 \pm 149,7$ & $321,1 \pm 173,0$ & 0,894 \\
& $\boldsymbol{v}_{e}$ & $0,7 \pm 0,3$ & $3,7 \pm 2,6$ & $10,1 \pm 9,1$ & 0,007 \\
& $\boldsymbol{v}_{p}$ & $0,5 \pm 0,1$ & $0,6 \pm 0,6$ & $1,3 \pm 2,2$ & 0,381 \\
\hline
\end{tabular}

Table 3.2. PK parameters results. Units: $K^{\text {trans }}$ and $k_{e p}(\mathrm{ml} / \mathrm{min} / 100 \mathrm{ml}), v_{e}$ and $v_{p}(\%)$.

\section{$\underline{\text { Reproducibility analysis }}$}

Except for the RMS_CoV for $v_{p}$, the other reproducibility values were good or very good [11] (range $=8-19 \%$ ), with $k_{e p}$ being the most reproducible parameter in both models (table 3.3).

\begin{tabular}{ccccc}
\hline Model & Parameter & Difference & \% Difference & RMS CoV \\
\hline \multirow{2}{*}{ 1 compart. } & $\boldsymbol{K}^{\text {trans }}$ & $1,2 \pm 1,4$ & $9,2 \pm 10,3$ & 9,9 \\
& $\boldsymbol{k}_{e p}$ & $14,9 \pm 5,6$ & $10,2 \pm 4,5$ & 8,2 \\
& $\boldsymbol{v}_{e}$ & $1,2 \pm 1,0$ & $17,1 \pm 18,3$ & 14,7 \\
& $\boldsymbol{K}^{\text {trans }}$ & $1,5 \pm 1,3$ & $13,8 \pm 10,9$ & 12,8 \\
\multirow{2}{*}{ compart. } & $\boldsymbol{k}_{e p}$ & $17,5 \pm 12,2$ & $10,2 \pm 7,5$ & 9,2 \\
& $\boldsymbol{v}_{e}$ & $1,8 \pm 1,2$ & $23,7 \pm 24,1$ & 19,0 \\
& $\boldsymbol{v}_{p}$ & $0,08 \pm 0,08$ & $46,5 \pm 58,5$ & 262,1 \\
\hline
\end{tabular}

Table 3.3. Reproducibility results. Units: $\mathrm{K}^{\text {trans }}$ and $\mathrm{k}_{\mathrm{ep}}(\mathrm{ml} / \mathrm{min} / 100 \mathrm{ml}) ; \mathrm{v}_{\mathrm{e}}, \mathrm{v}_{\mathrm{p}}$ and $\mathrm{RMS} \mathrm{CoV} \mathrm{( \% ).}$ 


\subsubsection{Discussion}

Patellar cartilage has not been an important focus of attention for the PK analysis of perfusion images, probably due to its low perfusion and the limitations of MR in spatial and temporal resolutions. However, some studies have pointed to a correlation between articular cartilage degeneration and the presence of an abnormality in its blood supply and nutrition [47].

This study seemed to be one of the first evaluations of the perfusion and PK characteristics of the synovium articular cartilage, both in the normal and disease status. The DCE-MRI quantification by PK analysis showed a significant difference in the $K^{\text {trans }}$ and $v_{e}$ between normal cartilage and cartilage affected by chondromalacia and osteoarthritis. These values showed a surrogate index of an increase of microvascularity and interstitial volume as the cartilage degenerates to more advanced stages.

It has been also demonstrated that the results obtained considering or not the vascular contribution are different. This difference in the measurements is bigger as the degeneration of the cartilage increases, due to a more advanced grade of angiogenesis. From this experience, it is suggested not to include $\mathrm{v}_{\mathrm{p}}$ in the model, so that reproducibility and model stability increase. This one-compartment analysis may also decrease unnecessary complexity and enlarge the statistical difference between cartilage disease states.

This study has also demonstrated that the methodological reproducibility is good, with the only exception of the vascular volume fraction. It was found that $k_{\text {ep }}$ values had an excellent reproducibility, though they did not show differences to be used in cartilage tissue characterization. In longitudinal studies, DCE-MRI PK analysis could be used to measure the effectiveness of the treatment as the reproducibility of the methods has been properly assessed.

In this study, several assumptions were made. Cartilage has a low perfusion, there is a linear conversion between intensity and concentration, a direct use of 
the concentration curves can be employed without mathematical exponential approximation [10,17] and there is no need for dynamic image registration. Moreover, only the widest patellar transverse slice image was analyzed in each patient to improve the quality of the manual segmentation of the cartilage. Although this single analysis may be representative of the cartilage general state, a more detailed study should comprise the analysis of the whole cartilage to avoid missing partial distributions of the disease. Also, it is necessary to consider the bias of not having pathological proof of the normal cartilage and of some cases with degeneration.

In conclusion, it has been demonstrated that the PK analysis of the patellar cartilage presents statistical difference in the capillary permeability and interstitial component between normal and disease status. Even more, these abnormalities are higher with more diseased stages. Therefore, it seems reasonable to include more patients in future studies for better assessments.

\subsection{Assessment of femoropatellar cartilage degeneration in $3.0 \mathrm{~T}$}

\subsubsection{Results}

Statistically significant differences were found for $K^{\text {trans }}(1.7 \pm 2.0,4.9 \pm 5.0,10.0 \pm 11.5$ $\mathrm{ml} / \mathrm{min} / 100 \mathrm{ml}, p=0.002$, for normal, initial and advanced degeneration, respectively) and $v_{e}(2.0 \pm 2.0,4.5 \pm 2.2,8.4 \pm 7 \%, p<0.001)$ in the patellar cartilage, and for $K^{\text {trans }}(3.1 \pm 2.3,4.6 \pm 4.0,10.2 \pm 8.8, p=0.008)$ in the femoral cartilage (figure 3.3).

The Student-Newman-Keuls post-hoc test could discriminate advanced degeneration in all cases, but not between normal and initial degeneration. 

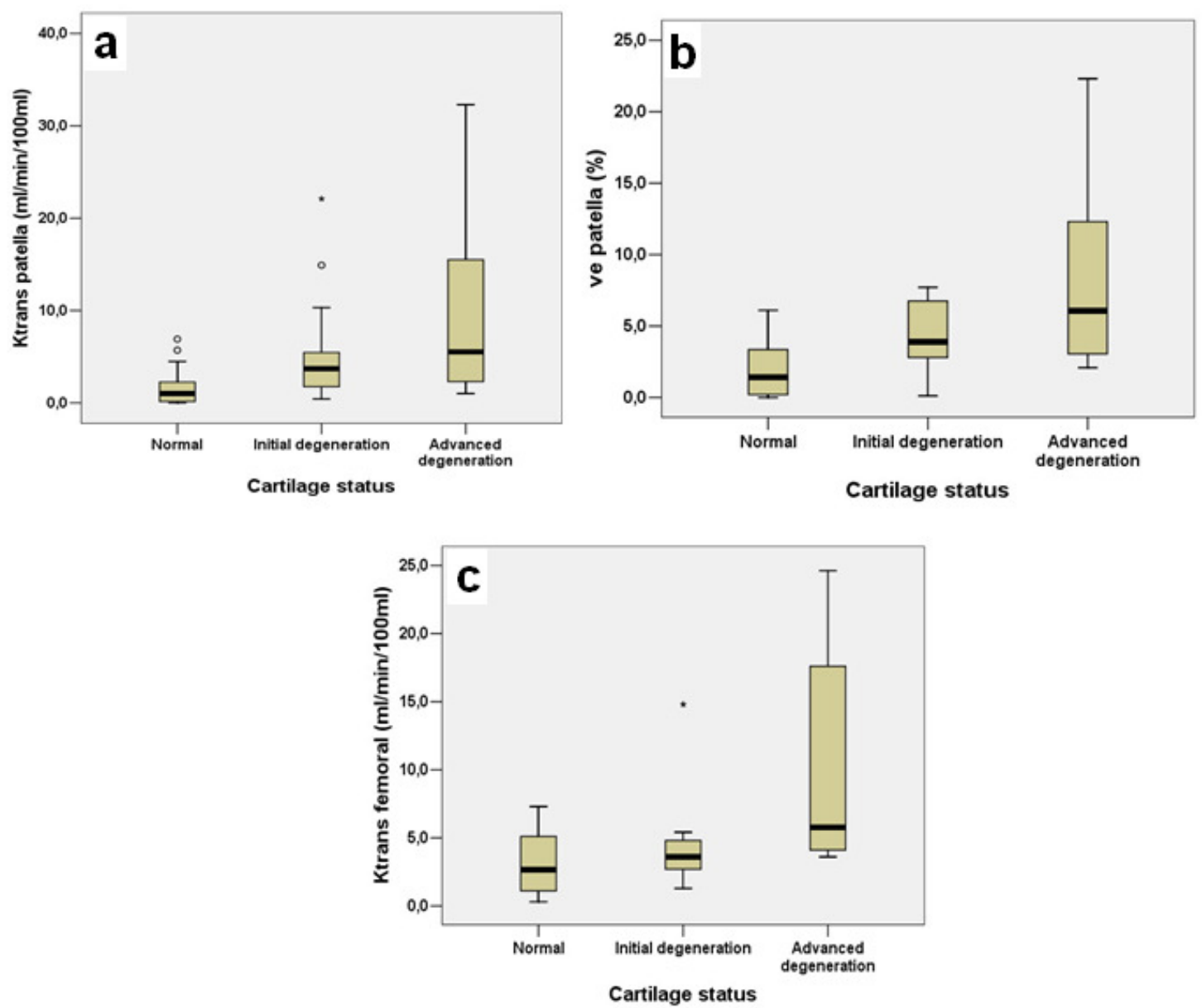

Figure 3.3. (a) Box-plots showing the differences in $K^{\text {trans }}$ and (b) $v_{e}$ for the patelar cartilages, and (c) Ktrans for the femoral cartilages.

\subsubsection{Discussion}

It was shown that as cartilage degeneration progresses, there is an increase in its vascularization when analyzed in a $3.0 \mathrm{~T}$ system. PK parameters, specifically capillary permeability and interstitial volume fraction, can be used as surrogate image biomarkers of disease progression. These results agree with those obtained in the previous study on a $1.5 \mathrm{~T}$ magnet. Neoangiogenesis maps may be useful in the diagnosis and follow-up of arthritis. 
The applied PK model dismisses vascular contribution, as cartilage is a naturally avascular tissue. Therefore, a specific term to model a highly vascularized situation was assumed unnecessary. Further studies should try to assess cartilage degeneration in earlier stages.

In conclusion, PK parameters obtained from 3.0 T DCE-MRI are able to depict the neovascularity associated to cartilage degeneration.

\subsection{Evaluation of glucosamine sulfate for the osteoarthritis treatment}

\subsubsection{Results}

Glucosamine sulfate treatment was considered clinically effective. Statistically significant differences were found in both the pain and the functional outcome scores for patients under glucosamine compared with the control group. Treatment with glucosamine sulfate reduced patients pain and improved their knee function compared with the controls ( $p<0.01$ for both clinical indexes). Table 3.4 summarizes the results for the clinical evaluations before the first MR study and 6 months afterwards.

\begin{tabular}{|c|c|c|c|c|c|}
\hline & \multicolumn{2}{|r|}{ Controls } & \multicolumn{2}{|c|}{ Glucosamine sulfate } & \multirow[b]{2}{*}{$p$} \\
\hline & Baseline & 6 months & Baseline & 6 months & \\
\hline VAS & $6.8 \pm 0.4$ & $7.0 \pm 0.0(3.3 \pm 7.4 \%)$ & $7.3 \pm 1.1$ & $\begin{array}{c}3.6 \pm 1.3 \\
(-51.8 \pm 12.3 \%)\end{array}$ & $<0.001$ \\
\hline$A K S$ & $26.0 \pm 5.5$ & $26.0 \pm 5.5(0 \pm 0 \%)$ & $18.6 \pm 6.9$ & $\begin{array}{c}42.9 \pm 2.7 \\
(165.5 \pm 114.6 \%)\end{array}$ & $<0.01$ \\
\hline
\end{tabular}



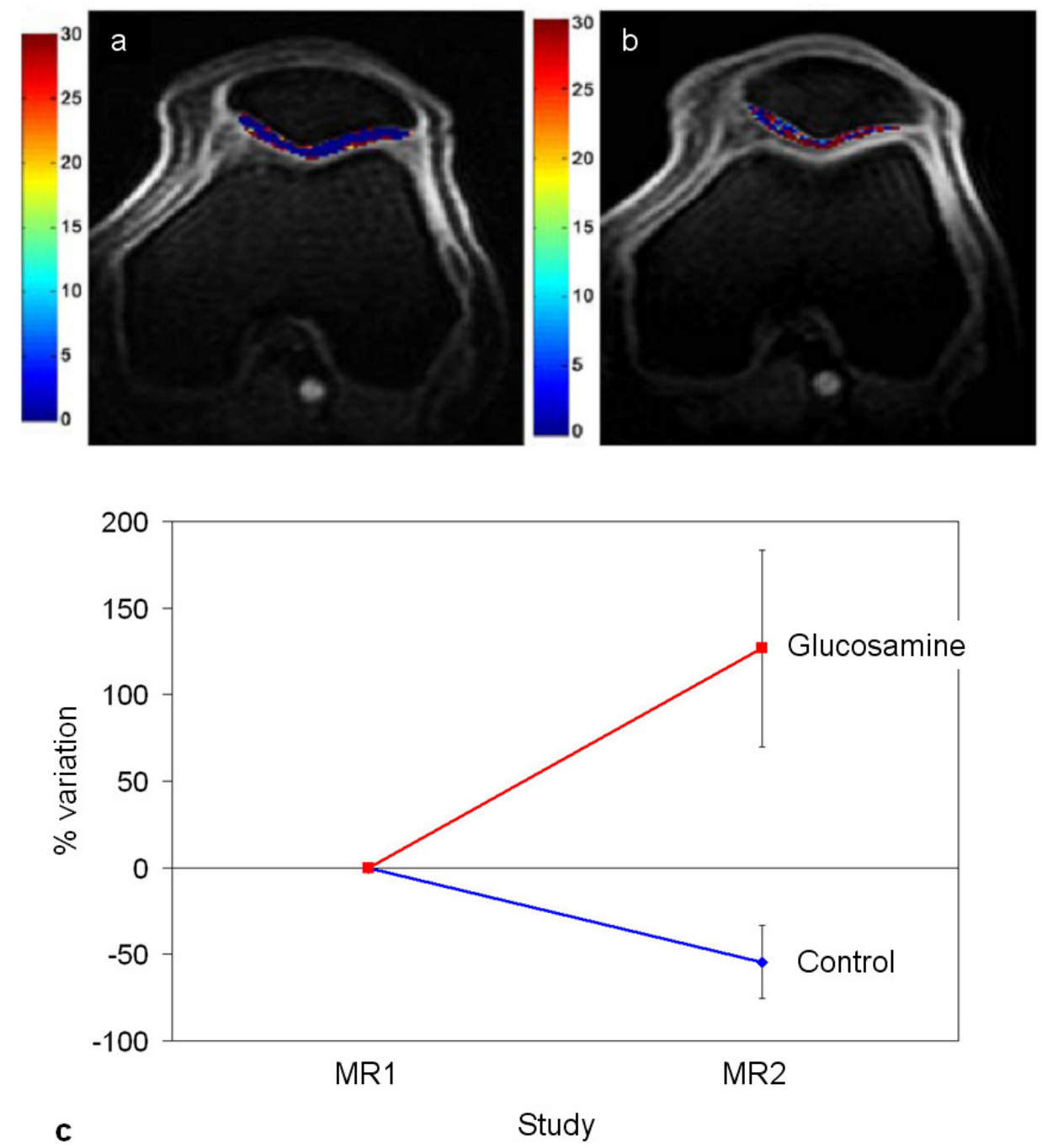

Figure 3.4. Cartilage parametric maps for $K^{\text {trans }}$ in a patient with osteoarthritis for the first (a) and second (b) MR examinations. There is an increase in the values of permeability. (c) \% difference in $K^{\text {trans }}$ for all patients (glucosamine and controls) and for both chondromalacia and osteoarthritis.

In the PK analysis, there was a statistically significant difference in the controls vs. glucosamine for $K^{\text {trans, }}$ with higher values in the glucosamine sulfate group ($54.4 \%$ vs. $126.7 \%$, controls vs. glucosamine sulfate, respectively) (figure 3.4 ). The $k_{e p}$ differences were not statistically different. Regarding $v_{e}$, there was a trend towards higher differences in percentage in the glucosamine sulfate group $(p=0.11)$. Table 3.5 summarizes the values of the parameters and the differences in the MR-calculated parameters between the two MR examinations. 


\begin{tabular}{|c|c|c|c|c|c|}
\hline Parameter & Group & MR1 & MR2 & Difference (\%) & $p$ \\
\hline \multirow{2}{*}{$K^{\text {trans }}$} & Controls & $17.1 \pm 7.1$ & $12.5 \pm 3.2$ & $\begin{array}{c}-54-4 \pm 21.2 \\
(9.5)\end{array}$ & \multirow{2}{*}{$\mathrm{p}<0.001$} \\
\hline & Glucosamine & $9.1 \pm 6.5$ & $14.4 \pm 8.3$ & $\begin{array}{c}126.7 \pm 56.9 \\
(21.5)\end{array}$ & \\
\hline \multirow{2}{*}{$k_{e p}$} & Controls & $367.0 \pm 130.5$ & $314.5 \pm 237.3$ & $\begin{array}{c}4.6 \pm 96.2 \\
(43.0)\end{array}$ & \multirow{2}{*}{$p=0.76$} \\
\hline & Glucosamine & $287.9 \pm 342.4$ & $373.4 \pm 130.4$ & $\begin{array}{c}-11.7 \pm 85.1 \\
(32.2)\end{array}$ & \\
\hline \multirow{2}{*}{$v_{e}$} & Controls & $14.2 \pm 11.4$ & $9.3 \pm 2.7$ & $\begin{array}{c}-36.1 \pm 90.8 \\
(40.6)\end{array}$ & \multirow{2}{*}{$p=0.11$} \\
\hline & Glucosamine & $9.3 \pm 6.2$ & $20.4 \pm 16.1$ & $\begin{array}{c}118.4 \pm 204.7 \\
(77.4)\end{array}$ & \\
\hline
\end{tabular}

Table 3.5. Summary of the results for the difference in the main PK parameters in both proposed models. The values are shown as mean \pm standard deviation (standard error of the mean). Units: $K^{\text {trans }}$ and $k_{e p}(\mathrm{ml} / \mathrm{min} / 100 \mathrm{ml}), v_{e}(\%)$.

The power calculation curve for $K^{\text {trans }}$, obtained for the worst case (highest standard deviation, $56.9 \%$ for the glucosamine group, alpha=0.05, $n 1=10$ and $n 2=6$ ), showed a value of 0.64 for a difference of $72.3 \%$ (mean percentage difference between glucosamine and controls). For $V A S$, the power calculation (highest standard deviation, $12.6 \%$, alpha $=0.05, n 1=10$ and $n 2=6$ ) showed a value of near 1 for a difference of $55.1 \%$. For $A K S$ no power calculation was obtained since the control group had null standard deviation and the difference was $165.4 \%$.

\subsubsection{Discussion}

In an effort to increase the in-vivo knowledge of the cartilage status, MR imaging can be used to quantitatively assess different biomarkers. The most evaluated 
parameters are cartilage thickness and volume, delayed contrast-enhancement and the physical properties of the T2 and T1 relaxation times.

Mature articular chondrocytes in physiological condition are essentially devoid of vascular structure. However, blood vessel growth into the articular cartilage may contribute to cartilage damage. Different studies have pointed to a correlation between articular cartilage degeneration and the presence of an abnormality in its blood supply and nutrition [40-47,101]. Although diffusion from the synovium fluid into the cartilage matrix is a known mechanism for cartilage nutrition, a direct blood supply from the subchondral bone is observed in osteoarthritis patients $[118,119]$.

In this sense, DCE-MRI pharmacokinetically derived parameters can be used as a biosignature of cartilage degeneration. PK models focus directly on the tissue microvascular function, providing a reliable and reproducible measurement of angiogenesis and capillary membrane permeability properties. This technique, although lacking global standardization, is considered an accurate and validated neoangiogenesis biomarker even in complex models [120].

Reproducibility of the PK cartilage calculations for 1.5 Tesla acquisitions is high, with a very low test-retest root mean square coefficient of variation (9-19\% for $K^{\text {trans }, ~} 8-15 \%$ for $\mathrm{k}_{\mathrm{ep}}$ and $14-23 \%$ for $\mathrm{v}_{\mathrm{e}}$ ) [101,103]. In this series, influence of time and treatment was much larger than methodological variability (control group, root mean square coefficient of variation of $30 \%$ for $K^{\text {trans }}, 110 \%$ for $k_{e p}$ and $71 \%$ for $v_{e}$; glucosamine group, root mean square coefficient of variation of $76 \%$ for $K^{\text {trans }}, 98 \%$ for $k_{e p}$ and $98 \%$ for $\left.v_{e}\right)$. Even more, when compared with previous studies using the same methodology in different populations, all the results had a similar range of values [101].

This study showed a clear influence of glucosamine sulfate treatment in the PK results. There is a significant difference in $K^{\text {trans }}$ after glucosamine administration compared with the control group. Patients treated with glucosamine sulfate had an increment in their cartilage capillary permeability as evaluated after 6 months 
of medication. There was also an increment in the volume of the interstitial space $v_{e}$, but the difference was not significant, most probably due to the small number of cases and the large standard deviation. The $k_{e p}$ did not show any significant change between both examinations, in neither group of patients, due to the fact that the cartilage enhancement curves had very slow washout (the curve decay modeled with the $k_{e p}$ parameter). This means that $k_{e p}$ cannot be used as a biomarker to assess differences in cartilage status.

Glucosamine sulfate stimulates the synthesis of proteoglycans and, together with other biochemical properties, improves the quality of the cartilage matrix. As found in this study, glucosamine sulfate administration has an effect on the capillary properties of cartilage, at least if evaluated 6 months after treatment onset. Although a decrease in the angiogenesis parameter values with treatment was initially expected, the observed effect was the opposite: an increase in the cartilage vascular permeability after treatment. This result could be associated with an increment in the angiogenesis associated with the promotion of reparative cartilage processes and with the metabolic cascades linked to the drug, as this repairing process may be energy consuming.

As the 6-months PK evaluation seems to relate to the increased metabolic effect of glucosamine sulfate on cartilage, it seems reasonable that the curative effect of glucosamine sulfate should be evaluated over a longer period, most probably at least 12 months after starting the administration of the drug. In order to test if PK MR evaluates an increase of cartilage metabolism and perfusion in patients treated with glucosamine sulfate, another study will be required with different methodology and examination times to be confirmed.

As PK analysis of DCE-MR images may model the vascular and interstitial behavior of different pathological conditions in tissues, the obtained parameters in this study must be considered reproducible, as similar MR sequences, contrast injection protocols and measurement procedures were used. The fact that the molecular weights of glucosamine sulfate (456 Daltons) and the used gadolinium 
chelate (gadodiamide DTPA_BMA, Omniscan) (573 Daltons) are quite similar makes this PK modeling even more promising to evaluate the effect of glucosamine sulfate on the capillary properties of the diseased cartilage. If the finding of a significant increase in $K^{\text {trans }}$ in osteoarthritic patients receiving glucosamine sulfate is replicated in a larger series, this parameter could be considered a surrogate endpoint of treatment effect, reflecting most probably an increase of the microvascularity and membrane permeability in the glucosamine sulfate group. It has to be noted that the prescription formulation of glucosamine sulfate (1,500 mg once a day) was used, which has an established PK profile and clinical trial evidence. It is not known whether these results would be applicable to other glucosamine or glucosamine sulfate dietary supplement or generic preparations.

One bias of this study is the use of mean values to characterize cartilage. This whole analysis may hide the presence of degenerated regions whose values are minimized when averaged with the rest of the cartilage. Parametric maps, histograms or regional analysis and visualization tools should be used in order to depict abnormal regions and interpret local results. These representations will offer additional information on degeneration location, size and grading.

Also, the choice of the AIF for the PK study may affect the reproducibility among different sites, as other studies related to PK analysis use different input curves, such as population averaged or reference curves [14,106]. In this study, individual input curves were used, chosen directly from the popliteal artery in each case, as both the spatial and temporal resolutions of the acquisition sequence were high enough.

As cartilage degeneration is also associated with changes in synovium and subchondral vascularization, this may be a source of bias. Cartilage tissue was carefully segmented, excluding synovium and subchondral bone, making sure that only cartilage was included in the image analysis. Therefore, the contribution of other "non-cartilage" structures to the cartilage evaluation can be 
neglected. Also, as the differences between the two groups of patients were evaluated, the variability associated with the methodology should be present in both groups and cannot explain the different group results. The values obtained from the power calculation allow an acceptable degree of confidence in the results.

In conclusion, glucosamine sulfate decreases pain and improves the functional outcome in patients with cartilage degeneration. Glucosamine sulfate treatment has also an effect on the vascular properties and cartilage metabolism. The strong relationship between treatment and increment in the cartilage capillary permeability (Ktrans) allows proposing its measurement as a surrogate marker for the 6-months glucosamine sulfate effect on the degenerated cartilage metabolism.

\subsection{Characterization of grade-IV CNS astrocytomas}

\subsubsection{Results}

Significant differences were obtained among the three regions of interest for $C B V, C B F, K^{\text {trans }}$ and $v_{p f p}(\mathrm{p}<0.001)$, either using the mean, the median or all the distributions (figure 3.5). The other parameters did not show significant differences. $C B V$ was globally the best parameter to separate tumor, peritumoral area and healthy tissue.

The analysis of the histogram shape showed statistically significant differences for the kurtosis of $K^{\text {trans }}$ and $k_{e p}$, as well as for the skewness of $C B V, C B F, K^{\text {trans }}$ and $v_{p f p}$. These values were significantly different between the tumor and the healthy region, while no separation could be made for the peritumoral area. Most parameters showed higher kurtosis in the healthy region, in comparison to the peritumoral and the tumor (figure 3.6), a fact that is related to tumor heterogeneity. 

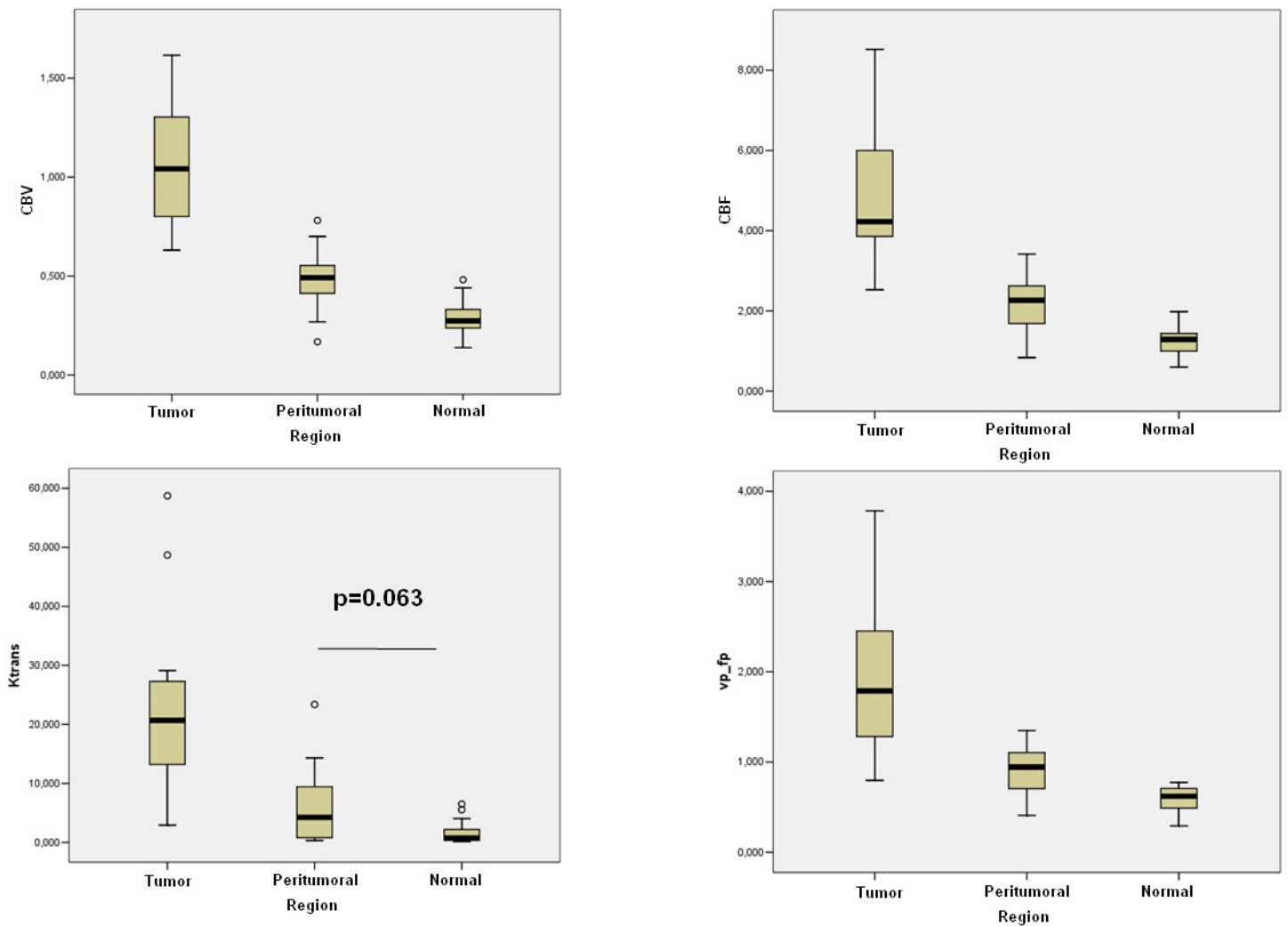

Figure 3.5. Box plots showing $\mathrm{CBV}, \mathrm{CBF}, \mathrm{K}^{\text {trans }}$ and $\mathrm{v}_{\mathrm{pfp}}$ for each region.
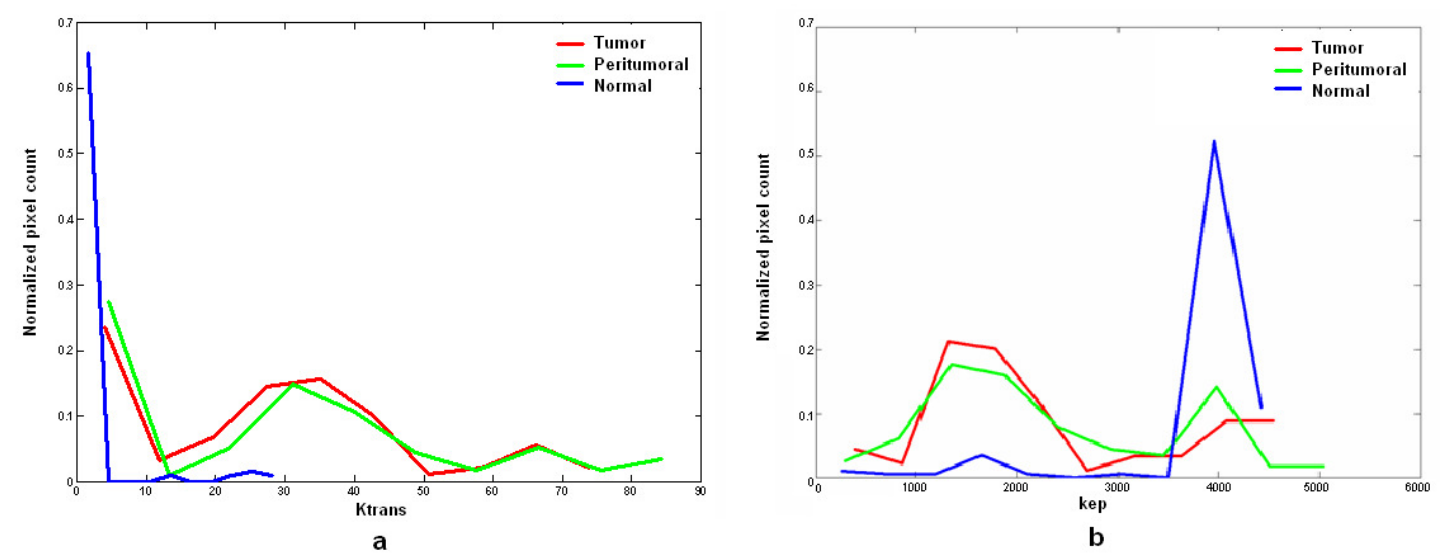

Figure 3.6. Example of normalized histograms for (a) $\mathrm{K}^{\text {trans }}$ and (b) $\mathrm{k}_{\mathrm{ep}}$. It can be seen that the kurtosis is much higher in the normal region than in the tumor and the peritumoral areas. 

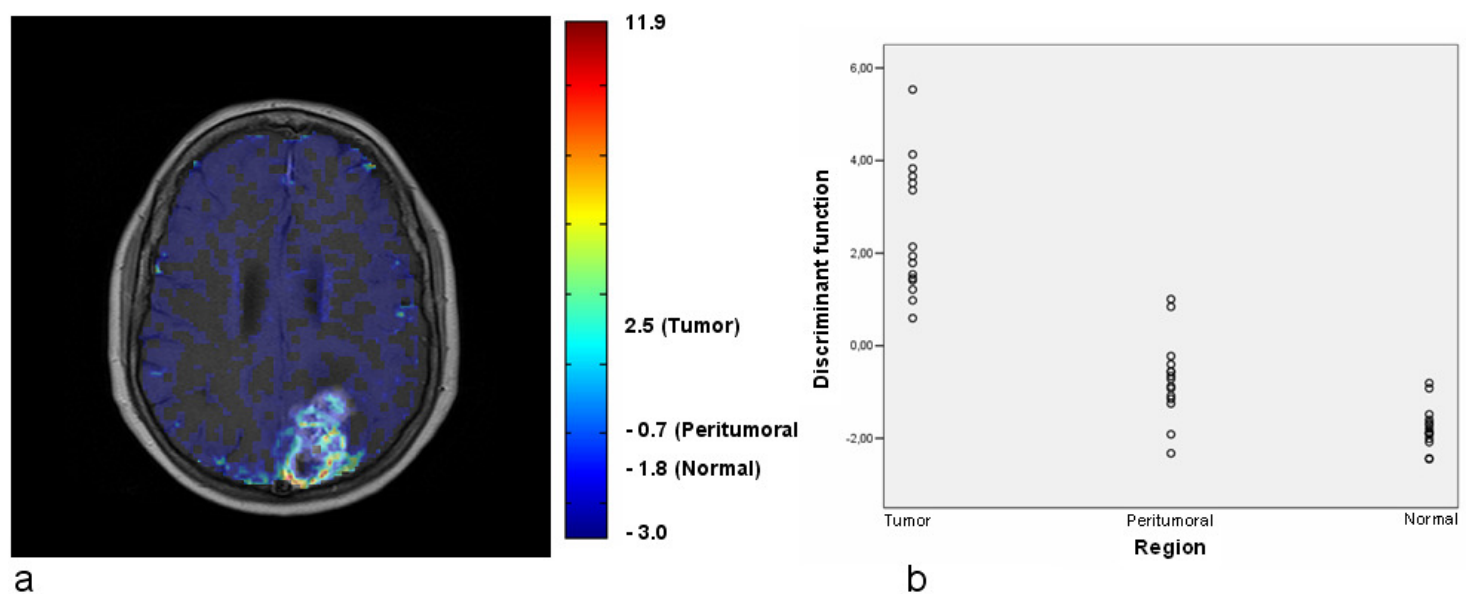

Figure 3.7. (a) Nosologic image obtained from the discriminant function. (b) Results of the discriminant function for each region. It can be seen that tumor and normal tissue can be perfectly separated. However, there are some peritumoral regions which are incorrectly classified. This may be due to mistakes in the manual selection of the ROIs, which are related to the infiltrating properties of these tumors.

The best discriminant function was obtained for a combination of $K^{\text {trans }}$ and $C B V$, with an $84.4 \%$ of the regions classified correctly: $\mathrm{F}=0.054 \cdot K^{\text {trans }}+3.933 \cdot C B V-3$ (with centroids in $2.482 /-0.716 /$-1.766, for tumor, peritumoral and normal areas, respectively). To obtain this function the whole distribution of values and the mean were used. This function classified correctly 14 tumor regions (1 was interpreted as peritumoral), 11 peritumoral ( 2 were interpreted as tumors and 2 as normal) and 13 normal (2 were interpreted as peritumoral). These classification results are slightly better than when $C B V$ was used alone to classify the regions (78\% classified correctly). Finally, a series of nosologic images were obtained from the discriminant function (figure 3.7).

\subsubsection{Discussion}

Most studies focusing on the differentiation of tumor grades have used the $C B V$ or the relative $C B V$ (normalized by the contralateral white matter). This method is widely used but also has some limitations, especially regarding the selection of ROIs. In an attempt to overcome this, the use of histograms has been proposed, 
with better results [111]. The heterogeneous spatial distribution of the results has been used to differentiate tumor grades, especially between grade-III and gradeIV. The aim of this study was not differentiating tumor grades, as all of them where proved grade-IV, but to improve the differentiation of the different regions. This was achieved by the use of discriminant functions, which combine several parameters in order to improve the statistical power of each individual variable.

When the parameters were studied separately, the best results were found for $C B V$. The relationship between tumor and healthy region for the $C B V$ was approximately 3.5, which is a little lower than other published results. This may be due to the use of the whole tumor region, instead of smaller ROIs which are focused only on the maximum values [121].

The standard MR protocol used to calculate the PK parameters used a T1weighted sequence. In this work, it has been assumed that the contrast media concentration could also be calculated from a dynamic susceptibility-contrast sequence [122]. Although it is not possible to visualize the same effects with both sequences, the obtained $K^{\text {trans }}$ values are in accordance with those published in the literature, and could be used to differentiate the three regions properly.

The kurtosis results presented in this study agree with those of previous studies [111], where the heterogeneity is directly related to tumor aggressiveness.

The main limitation of the study is the way regions are selected. It is often extremely difficult to separate regions clearly, due to the infiltrating characteristics of the glioblastomas. This causes margins to be located beyond the visual margins of the images, a fact that may be leading to some mistakes in the results. 


\subsection{Magnetic field strength influence}

\subsubsection{Results}

\section{$\underline{\text { PK analysis }}$}

Statistically significant differences were observed between the 1.5 and $3.0 \mathrm{~T}$ groups for Ktrans (mean $\pm \mathrm{SD} ; 5.44 \pm 2.27$ vs. $1.01 \pm 0.41$, respectively; $p=0.005$ ) and $v_{p}(2.93 \pm 1.30$ vs. $0.75 \pm 0.64 ; p=0.007)$ (figure 3.8$)$. There were no significant differences for $k_{e p}(658.21 \pm 275.61$ vs. $410.89 \pm 204.06 ; p=0.08)$ and $v_{e}(5.03 \pm 5.83$ vs. $0.81 \pm 0.80 ; p=0.14$ ) values, although a clear trend was observed.
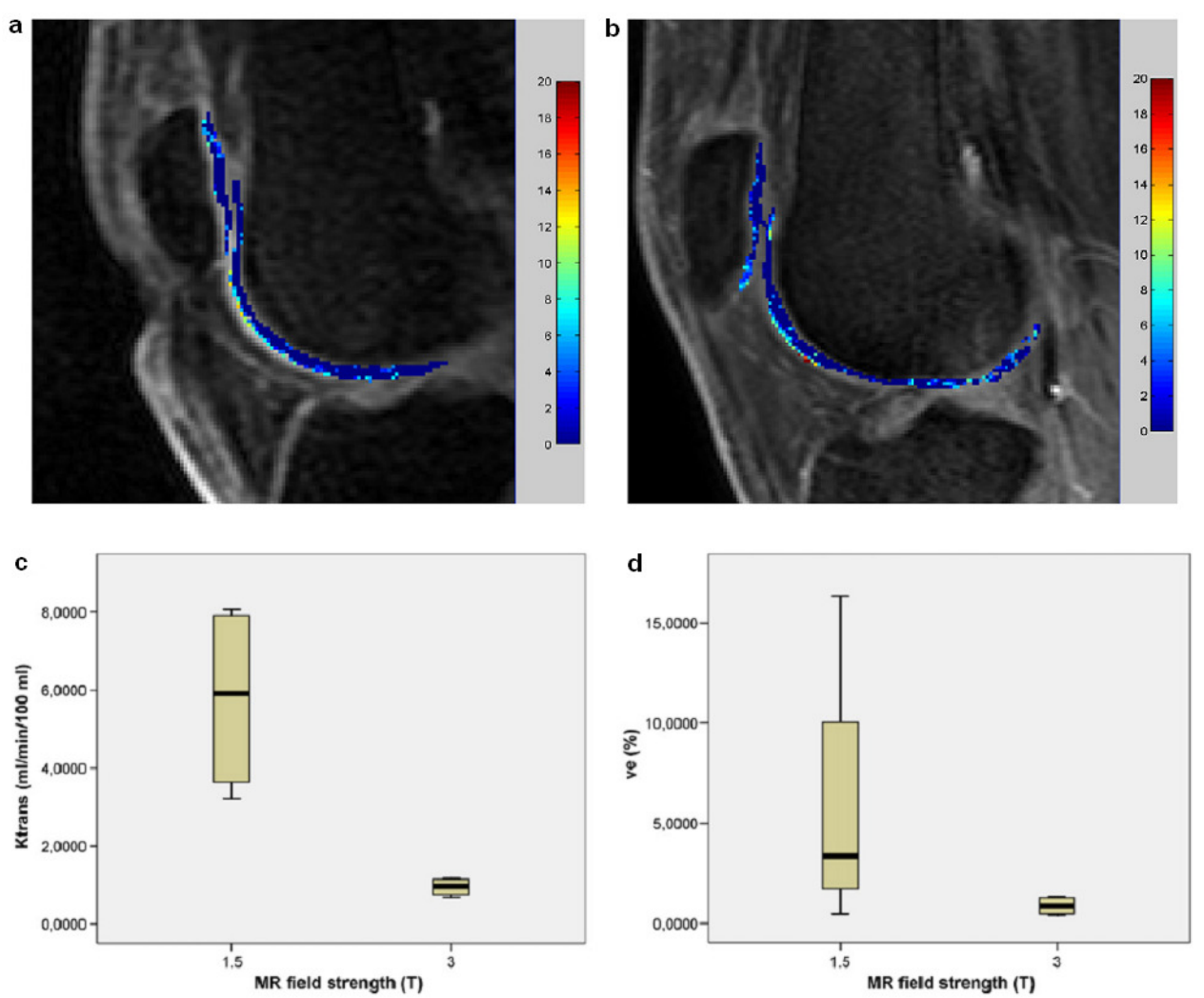

Figure 3.8. DCE-MRI sagittal slices showing the segmented cartilage with overlaid $K^{\text {trans }}$ values in (a) $1.5 \mathrm{~T}$ and (b) $3.0 \mathrm{~T}$. Box-plots for (c) $K^{\text {trans }}$ and (d) $v_{e}$ comparing the influence of the MR field strength on these parameters

When the same subject controls were evaluated in both 1.5 and 3.0 T equipments, a difference in $K^{\text {trans }}$ was also present $(5.77 \pm 2.49$ vs. $0.95 \pm 0.24 ; p=0.030)$. 
However, there were no significant differences for $k_{e p}(510.65 \pm 190.60$ vs. $595.54 \pm$ $55.61 ; p=0.42), v_{e}(5.89 \pm 7.11$ vs. $0.86 \pm 0.46 ; p=0.25)$ and $v_{p}(4.88 \pm 3.54$ vs. $1.27 \pm$ $0.32 ; p=0.09)$ values.

\section{Reproducibility analysis}

All the examinations were analyzed twice, the second time 7 days after the first analysis. The ROIs for the popliteal artery and the cartilage were manually drawn again by the same researcher. Reproducibility of the PK calculations, assessed in the 24 acquisitions, showed a test-retest root mean square coefficient of variation of $0.13,0.10,0.23$ and 0.18 for $K^{\text {trans }}, k_{e p}, v_{e}$ and $v_{p}$, respectively. Except for $v_{e}$, the values of reproducibility were good or very good [43], being $k_{e p}$ the most reproducible parameter with a $10 \%$ coefficient of variation.

Comparing field strength, reproducibility for $1.5 \mathrm{~T}$ showed values of $0.19,0.15$, 0.23 and 0.15 for $K^{\text {trans }}, k_{e p}, v_{e}$ and $v_{p}$, respectively, and for $3.0 \mathrm{~T}$ values of $0.10,0.06$, 0.24 and 0.20 for $K^{\text {trans }}, k_{e p}, v_{e}$ and $v_{p}$, respectively.

\subsubsection{Discussion}

At the time of this study, all previous PK studies had been obtained from $1.5 \mathrm{~T}$, so the influence of the main magnetic field strength in the PK parameters extracted from DCE-MRI of the patellar cartilage was unknown. If field strength modifies the normal range values, these should be given separately to be used clinically. Even more, the reproducibility of the intrasubject results needs to be assessed to fully define this biomarker.

In this study, it was demonstrated that the PK variables are in fact influenced by field strength, at least between $1.5 \mathrm{~T}$ and $3.0 \mathrm{~T}$. The reproducibility from this study is similar to previous results [46], at least regarding methodology. However, other factors such as the influence of the radiofrequency pulses in different equipments, the contrast media or the use of different AIFs, remain 
unclear. Future studies should focus on the analysis of not only the magnetic field strength, but also these other factors which may probably provide additional variability to the PK analysis of DCE-MR images.

If this PK analysis has to be used in longitudinal studies for drug trials, the effectiveness of the treatment has to be larger than the normal fluctuations in methodological reproducibility. PK parameters are reproducible as long as similar field strengths, sequences and image processing techniques are used.

In conclusion, we have demonstrated that although the PK analysis of the patellar cartilage may be used as a biomarker of the capillary permeability and interstitial component of the cartilage, it is influenced by the field strength. Overall, 3.0 $\mathrm{T}$ analysis shows more consistent and reproducible calculations. Therefore, the range of normal values of these parameters should be adjusted to the field strength.

\subsection{Assessment of the arterial contribution in liver carcinomas}

\subsubsection{Results}

All the parameters showed a clear differentiation between liver and tumors in the parametric colored maps (fig. 3.9). The $A$ I maps showed a wider value range inside the lesion in comparison with the $v_{p}$ maps, a fact probably related to the greater sensitivity of $A I$ to tumor heterogeneity (fig. 3.9a and 3.9f).

The mean \pm SD and the median for the liver and the tumor are shown in table 3.6. There were statistically significant differences between liver and tumor for all the parameters. The $A I$ showed much higher values than $v_{p}$ for both the tumor and the liver. 

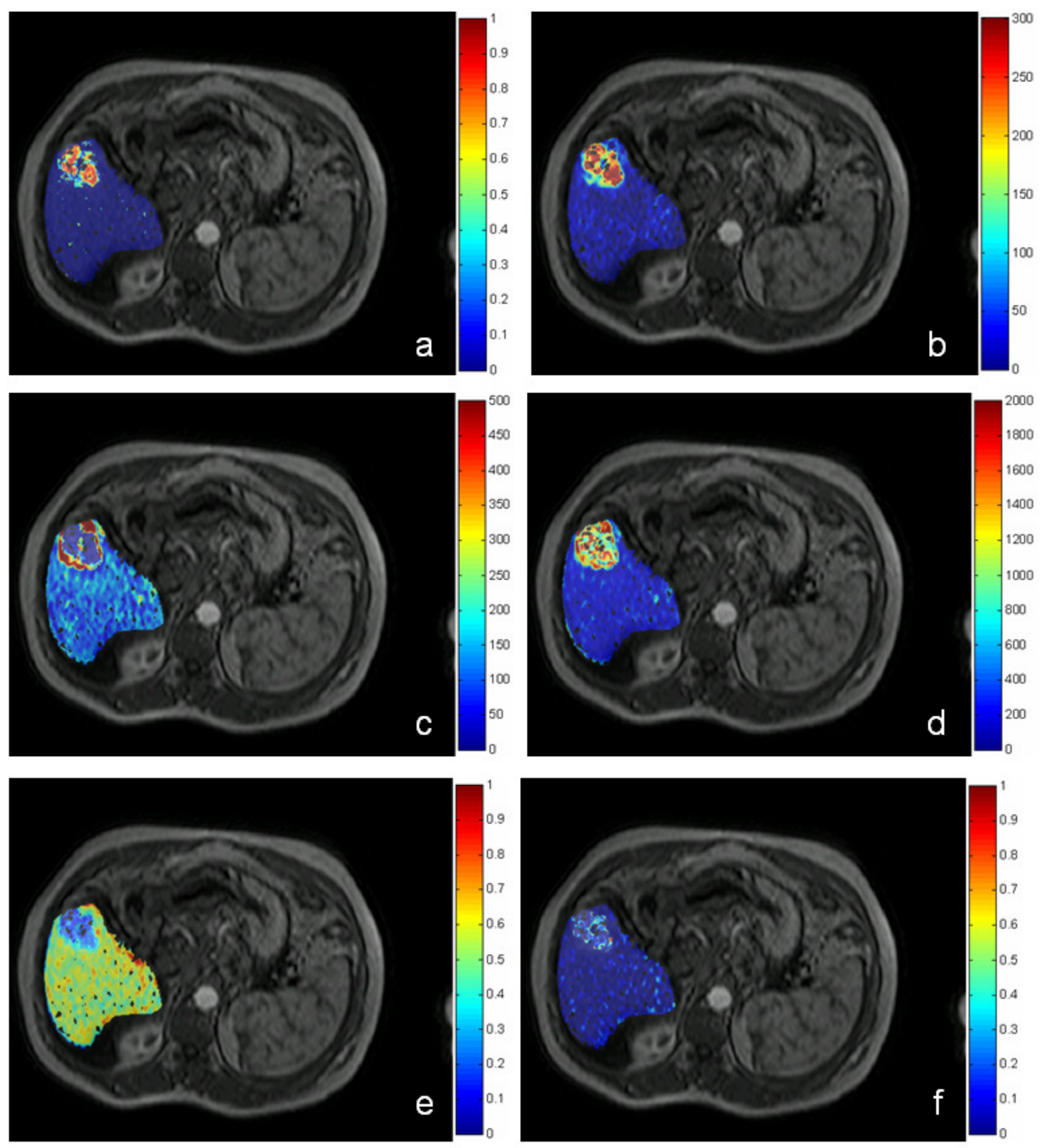

Figure 3.9. Parametric colored maps for the calculated parameters: (a) $A I$, (b) $K^{\text {trans1 } 1}$, (c) $K^{\text {trans2 }}$, (d) $k_{e p}$, (e) $v_{e}$ and (f) $v_{p}$. Units: $A I$ and $v_{p}$ (no units), $K^{\text {trans } 1}(\mathrm{ml} / \mathrm{min} / 100 \mathrm{ml})$. 


\begin{tabular}{cccccccc}
\hline Tissue & $\boldsymbol{N}$ & $\boldsymbol{K}_{\text {trans1 }}$ & $\boldsymbol{K}_{\text {trans } 2}$ & $\boldsymbol{k}_{e p}$ & $\boldsymbol{v}_{e}$ & $\boldsymbol{A I}$ & $\boldsymbol{v}_{p}$ \\
\hline \multirow{2}{*}{ Liver } & 15 & $13.7 \pm 11.3$ & $175.8 \pm 158.4$ & $348.0 \pm 209.8$ & $0.49 \pm 0.09$ & $0.12 \pm 0.26$ & $0.02 \pm 0.05$ \\
& & $(11.5 \pm 11.7)$ & $(181.3 \pm 169.9)$ & $(349.2 \pm 217.7)$ & $(0.50 \pm 0.09)$ & $(0.00 \pm 0.05)$ & $(0.01 \pm 0.00)$ \\
Tumor & 15 & $151.5 \pm 36.4$ & $232.8 \pm 176.5$ & $1869.2 \pm 1950.0$ & $0.37 \pm 0.06$ & $0.56 \pm 0.24$ & $0.04 \pm 0.05$ \\
& $(149.5 \pm 33.9)$ & $(17.9 \pm 34.5)$ & $(562.0 \pm 134.3)$ & $(0.37 \pm 0.07)$ & $(0.57 \pm 0.21)$ & $(0.01 \pm 0.00)$ \\
$\mathbf{p}_{\text {mean }}$ & 15 & $<0.001$ & 0.481 & 0.033 & 0.005 & $<0.001$ & 0.472 \\
$\mathbf{p}_{\text {median }}$ & 15 & $<0.001$ & 0.021 & 0.024 & 0.003 & $<0.001$ & 1.000 \\
\hline
\end{tabular}

Table 3.6. Mean values for tumor and liver. Values are mean \pm standard deviation, with the median between brackets. (Units: $K^{\text {trans } 1}, K^{\text {trans } 2}, k_{e p}: \mathrm{ml} / \mathrm{min} / 100 \mathrm{ml} ; v_{e}, A I$ and $v_{p}$ : no units).

Correlation coefficients were poor $(r<0.5)$ for $A I-v_{p}(r=0.062$ and $r=-0.083$, for liver and tumor, respectively) and $A I-K^{\text {trans } 1}(r=0.143$ and $r=0.440$, for liver an tumor, respectively). After plotting each pair of values it could be seen that there was not a patent linear relationship between $A I$ and $v_{p}$, and between $A I$ and $K^{\text {trans1, }}$ neither in the liver nor in the tumor (fig. 3.10).
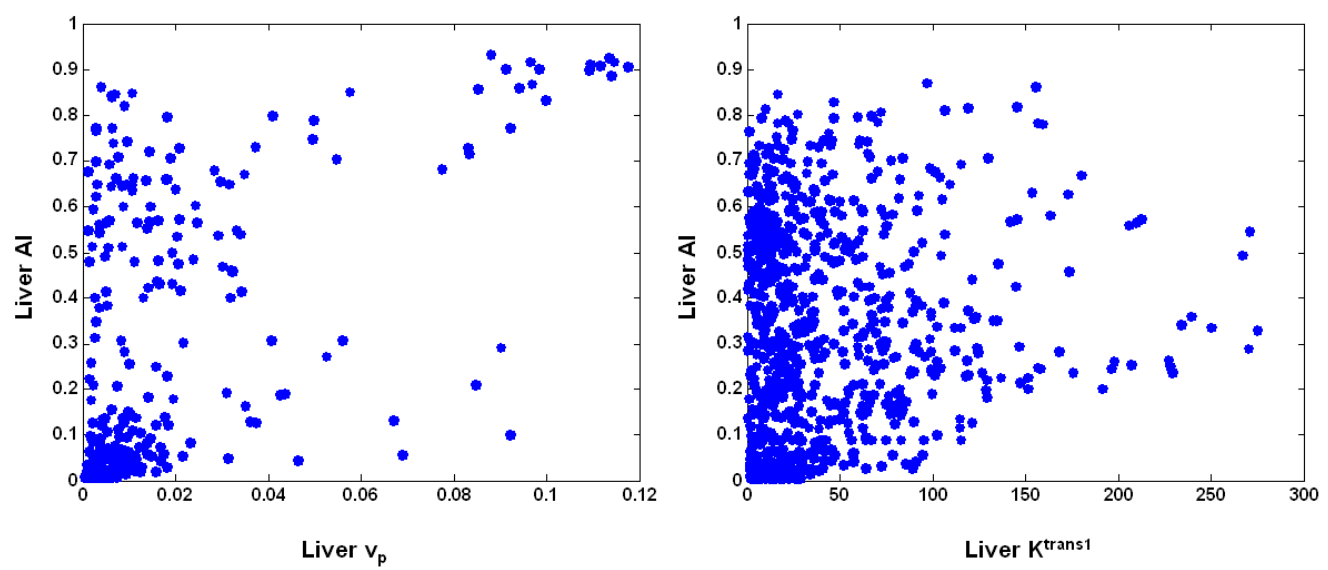

(a)
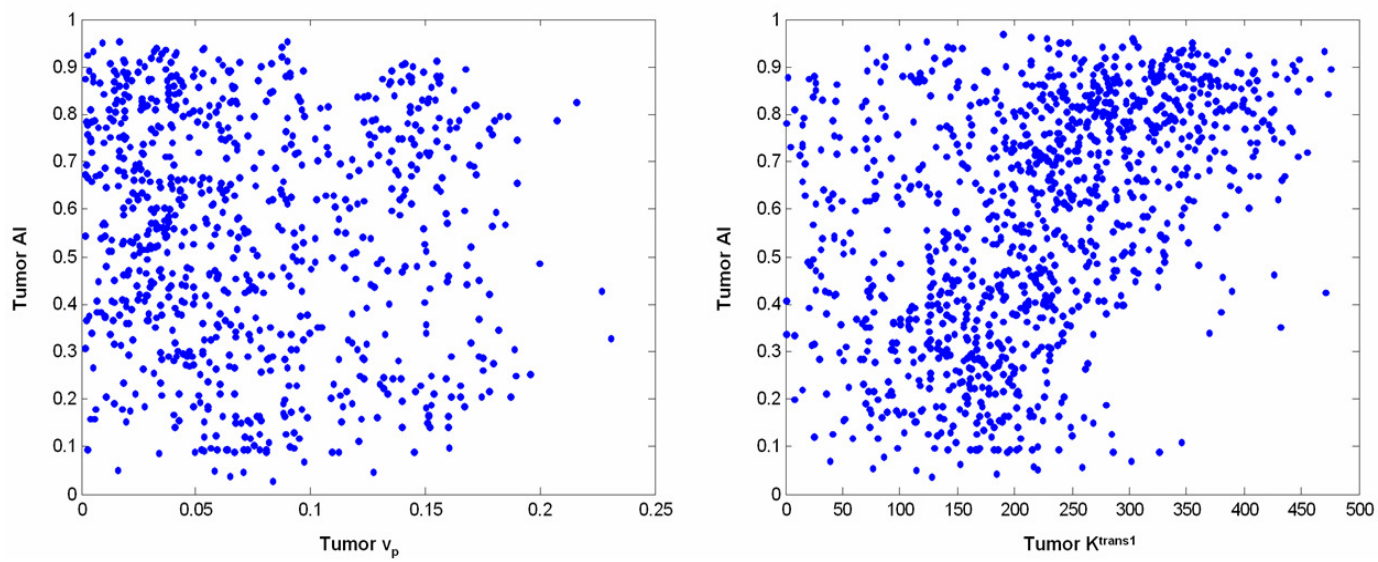

(b)

Figure 3.10. Dispersion plots comparing the values of the comparisons between $A I$ vs. $v_{p}$ and $A I$ vs. $K^{\text {trans1 } 1}$, for one representative case: (a) liver and (b) tumor. Units: $A I$ and $v_{p}$ (no units), $K^{\text {trans1 }}$ $(\mathrm{ml} / \mathrm{min} / 100 \mathrm{ml})$. 


\subsubsection{Discussion}

This work has focused on the quantification of neoangiogenesis and neovascularization in hepatocellular carcinoma lesions from DCE-MR images. One of the main characteristics of tumors development is the formation of new vascular structures with chaotic architectures [23]. In comparison to the normal tissue, these structures show highly heterogeneous microvascular properties, as it can be seen from the pharmacokinetic analysis of DCE-MR images, where permeability, washout rate, interstitial volume and vascular volume can be quantified. The MR capability to quantify these parameters with both high spatial and temporal resolutions allows using voxel-based analysis where, apart from mean regional values, heterogeneity can also be assessed at the tumor and the peritumoral regions with great accuracy [123]. In the widely used generalized pharmacokinetic model, the vascular term $\mathrm{v}_{\mathrm{p}}$ is introduced in order to quantify a significant arterial contribution to the enhancement curve of a tissue [69]. However, as it has been shown, a scaling factor such as $v_{p}$ may not be enough to characterize the dispersion of the AIF bolus. In fact, this is not caused by a limitation of the model, but by the need of using relatively distant arteries as input functions to the tissue of interest (such as the aorta in the study of liver perfusion). This choice introduces a bias which needs to be taken into account when analyzing any tissue. Ideally, several different smaller arteries should be used to study the whole liver, depending on their distance to the voxels under study. However, nowadays it is not possible to achieve such high spatial resolutions in DCE-MR clinical studies. Therefore, vascularization needs to be quantified in a different way, not as an absolute volume fraction, but as a relative measurement in comparison with the shape and the scale of the selected artery. Pharmacokinetic modeling of the liver is characterized by the use of a dual input, as the liver receives blood both from the hepatic artery and the portal vein. This property introduces further complexity in the model, as two input functions 
need to be introduced to fit the tissue response curves. However, if certain conditions are met, this dual input does not add any problem to characterize the arterial contribution. If the temporal resolution is high enough it is not difficult to separate aorta and portal peaks in time. In our study there were usually three or four dynamics between each peak, with enough time to detect any curve whose maximum was located during the arterial phase, i.e. after the aorta peak and before the portal peak. This criterion was always applied before any posterior operation on the curves, so that only voxels with significant arterial contribution were further processed to obtain the arterial index. At the same time, another criterion was applied to classify voxels into arterially and non-arterially significant. It consisted of comparing the maximum value of each voxel curve with the mean values plus three standard deviations of the second half of the curve. Although choosing the number of standard deviations is a relatively arbitrary decision, it proved to be robust enough to prevent any noisy curve to be interpreted as arterially significant.

The use of gradients to study changes in the curves suffered from some limitations, especially if the application handles noisy curves. Image registration can solve movement artifacts partially. In this study, non-rigid registration was used and the resulting images and the extracted curves were qualitatively better. Moreover, additional smoothing of the curves was considered necessary in order to make gradients more robust. However, it is known that smoothing tools reduce oscillations but also lower peaks which may be of interest (i.e. an arterial peak). To correct this undesired effect, when the curve peak was detected using the gradient, its smoothed values were substituted by the original ones, thus preserving the originally acquired arterial peak.

First pass phase curves were fitted with gamma probability density functions because of their particular shape. This gamma-based function is comprised of a bolus (similar to a Gaussian function) and a tail, which is appropriate to model 
the behavior of a fast rise and a fast (but slightly slower) decay of a first pass curve.

The comparison of the proposed $A I$ and the generalized kinetic model $\left(v_{p}\right.$ and with $\mathrm{K}^{\text {trans1) }}$ showed poor correlation values. As it has been stated, these parameters cannot be easily compared, as they represent different concepts, but some facts can be concluded: first, the AI showed a much wider range of values than $v_{p}$, a fact that suggests it can be used for a more specific assessment of lesion heterogeneity; and second, $A I$ provides a different information, as it can be seen from the lack of a clear linear relationship (fig. 3.10). In this study the Pearson correlation coefficient was used because it was expected to observe linear relationships between the variables. Other relationships should be studied in future studies including a larger cohort of patients.

Although $A I, v_{p}$ and $K^{\text {trans1 }}$ showed a bad correlation, it can be hypothesized that they are all modeling the first pass of the enhancement curve, especially when there is a significant arterial contribution. However, if the first pass phase is extracted from an enhancement curve (fig. 2.15), and the remaining washout curve is fitted to the generalized kinetic model, the resulting $K^{\text {trans1 }}$ is lower than that obtained using the whole enhancement curve. Therefore, it is difficult to assess how $K^{\text {trans } 1}$ models first pass, perfusion or permeability, three concepts which are different but clearly related.

In table 3.6 the median values of the distributions are also given. This statistic has the property of neglecting outliers, which can be easily obtained in complex fitting processes. Many studies related to pharmacokinetic modeling are now including the median values in their results because they seem to provide a more robust and coherent estimate of the behavior of a region. For instance, in table 3.6 it can be seen that if the mean is used, $K^{\text {trans } 2}$ increases in the tumor in comparison to the liver, while if the median is used it is much lower in the tumor. Another statistical approach which seems promising is the use of histograms to characterize regional heterogeneity, as histograms provide the possibility to 
analyze different data subgroups (such as maximum percentiles) and offer statistics directly related to the heterogeneity of the region (such as the kurtosis). The proposed $A I$ offers new information for the assessment of hepatocellular carcinoma, as it complements the parameters obtained from the traditional kinetic analysis (arterial permeability, venous permeability or arterial ratio) which have been used as imaging biomarkers for assessing tumor aggressiveness or treatment effectiveness. It has been demonstrated that this index can offer relevant information about the lesion heterogeneity, as it is especially sensitive to changes in the arterial phase. This could help to further characterize the vascularization structures of the tumor, though with obvious constraints due to spatial and temporal resolution limitations. Further studies with more patients and higher magnetic fields should be carried out. Also, the use of this new imaging biomarker for the assessment of antiangiogenic drugs should be validated.

In conclusion, a new method has been proposed to characterize hepatocellular carcinoma lesions from the arterial vascularization point of view. A new imaging biomarker has been calculated in order to quantify the degree of similarity of the tissue enhancement curves in comparison to the AIF, taking into account both the change of scale and the dispersion of the bolus.

\subsection{Assessment of region heterogeneities in hepatocarcinomas}

\subsubsection{Results}

Kurtosis showed significantly different values between the lesion and the normal parenchyma for $K^{\text {trans } 1}(2.4 \pm 0.7$ and $4.5 \pm 2.5$, respectively, $p=0.039)$. No significant differences were obtained for the rest of parameters though a tendency towards lower kurtosis values was observed for the lesion ROIs. 


\section{Results and discussion}

\subsubsection{Discussion}

Lesion heterogeneities in hepatocarcinoma lesions can be quantified using histogram analysis, particularly with the calculation of the kurtosis. Lower values of kurtosis were observed for every PK parameter when comparing lesion and normal parenchyma histograms. More cases and further histogram analysis techniques need to be assessed. 


\section{Future work}

\subsection{Arterial input function}

Several studies have shown the variability associated to the AIF selection $[80,124-$ 128]. Three different approaches are commonly taken:

- A reference AIF, modeled as a biexponential decay [72]

- An individual AIF, measured manually at some major vessel for each subject

- A population based AIF, obtained as a mean AIF from different individual AIFs

In [124] the individual AIF is proposed as the best solution to minimize variability and maximize the accuracy of patient-based results. Nowadays all MR equipments allow acquiring data at a high enough temporal resolution so that AIFs can be correctly sampled at almost every artery. A visual analysis of these AIFs will show that they present the usual pattern: baseline, fast rise, peak, fast decay, recirculation (especially in bigger arteries) and washout.

A much more interesting approach is that proposed in [81] where independent components analysis (ICA) is used to track the AIF from a major vessel into tissue smaller vessels, which will be irrigating the region of interest more directly. ICA is a signal processing technique developed to solve the problem blind source separation (BSS), which consists in extracting a series of signals (sources) which cannot be observed directly from another group of signals which are a mixture of the these sources. ICA assumes that any observed signal is a linear combination of independent sources [129].

Theoretically, a more accurate AIF can be obtained using ICA. However, the detection of smaller vessels depends on image spatial resolution and the presence of partial volume effects which may mix tissue and vessel into the same voxel, thus blurring the net vascular contribution of a small vessel. Moreover, the 
selection of big ROIs (such as in the study of the liver) may introduce difficulties when it comes to selecting which AIF is more appropriate for each part of the liver. Ideally, a different AIF should be selected for each voxel of the ROI under analysis, but the methodology proposed in [81] assumes a check point to select which AIF is likely to be used for the analysis from a series of possible curves, depending on the proximity to the ROI. So, if only the centroid of a ROI is used to measure AIF proximity, it may introduce important bias, underestimating or overestimating the AIF of each part of the ROI.

A possible solution is the division of the initial ROI into a series of subROIs so that the AIF of each subROI can be assessed separately. Again, this may be a slow process if the division demands many user interactions. But it can also be optimized if a multi-resolution strategy is followed. The proposed approach consists in estimating the initial AIF from the centroid of the ROI, then the ROI is split in two equal subROIs from which the centroid is also obtained. A new pair of AIFs is selected for each of the subROIs. If the AIFs are significantly different from the initial AIF then the subROIs are split again and the process is repeated until the selected AIF is no longer different. Finally, each ROI voxel is labeled according to its own AIF.

Although it may look like a long process, the AIF is not expected to vary so much, so the recursive process will most probably stop after a few subdivisions. Also, this modification should only be applied to those ROIs which are considerably big and which are expected to have several AIFs.

\subsection{PK modeling of kidneys}

DCE-MRI PK modeling of the kidneys presents an additional complexity to that presented in this thesis, as it is often modeled with a three-compartment model [130]: vessels, interstitial space and nephrons. This feature implies the necessity to include additional variables in the model, as well as further studies of the 
influence of each parameter. Up to now there are not many studies so, as a future application, it is proposed to study the nephrotoxicity of a radioactive drug (cisplanitum) in a longitudinal study with DCE-MRI.

\subsection{Multivariate image analysis}

Although a small application of MIA has been also presented in this thesis, more research on this field is required as it is a very promising approach for extracting further knowledge about the disease and how the combination of one-way analysis with multivariate techniques can improve the results.

\subsection{Analysis of curve fitting results}

The results presented in this thesis have been interpreted by experienced radiologists and the applications have demonstrated that they represented the physiological reality accurately. During the corresponding test phases for each application, all fittings were qualitatively assessed by the observation of the acquired and the fitted curves. Once it was seen that most curves were fitted correctly, the batch processes were released and all the cases were analyzed automatically. However, a more strict approach should focus on the study of the fitting residuals in order to study normality, possible local minima or lack of convergence.

\subsection{Methodological standardization}

As it has been already introduced in this thesis, one of the most important problems for a widespread application of DCE-MRI PK modeling is the lack of standardization both in acquisition and in post-processing methodologies. This 
prevents its use in multi-center trials or the publication of standard ranges of normality and disease.

There are currently some initiatives focused on the production of white papers for the use of PK parameters in the evaluation of antiangiogenic treatments [2]. Also, a new research institution, the European Institute for Biomedical Imaging Research (EIBIR) has recently been set up in order to lump together researchers, government and companies so that common efforts can be made.

\subsection{Comparison of DCE-MRI PK modeling with IVIM and ASL}

IVIM (intravoxel incoherent motion) and ASL (arterial spin labeling) are techniques which offer quantitative information about the perfusion without the need of an exogenous contrast agent. With the new hardware and software developments they are becoming more and more fashionable, as they provide a quantitative tool to assess perfusion without the risks associated to contrast media.

IVIM is based on MR diffusion weighted images (DWI) using several b-values sampled at a high rate in the lower range. In comparison to the traditional diffusion quantitation using the apparent diffusion coefficient (ADC), IVIM allows to separate the two different movements that water experiences in a voxel: a fast component, related to perfusion (low b-values), and a slow component, related to diffusion (high b-values). Therefore, the decay curve which relates signal intensity with the corresponding b-value can be separated into two parts using this equation [131]:

$$
\frac{S}{S_{0}}=(1-f) \cdot e^{-b \cdot D}+f \cdot e^{-b \cdot D^{*}}
$$

where $\mathrm{f}$ is the perfusion fraction, $\mathrm{D}$ is the extravascular diffusion component and $\mathrm{D}^{*}$ is the intravascular perfusion component. 
ASL is based on the magnetic marking of blood flow using radiofrequency pulses. Firstly, blood hydrogen nuclei are excited with radiofrequency pulses just before entering the region of interest, so that the longitudinal magnetization is inverted. Then, when excited blood enters the region of interest an image is acquired with the original magnetization of the tissue plus the blood magnetization. Finally, a second image is acquired without magnetic marking and a subtraction is performed, so only the magnetization corresponding to the initial magnetic marking remains and a perfusion image is provided. This technique is currently being implement in most new MR equipments.

These techniques are complex and have some limitations, but it is necessary to validate their performance in the clinical routine. A comparison of these techniques with the PK modeling of DCE-MR images is proposed in order to establish the reproducibility and accuracy of all those parameters which have similar physiological meaning.

\subsection{Development of an integrated post-processing clinical tool}

The software developed in this thesis has been implemented in Matlab as a tool designed for research purposes. Although it also has the possibility of transferring the results into a clinical report, its main aim was to be a benchwork for the study of the PK models.

Right now it has become a relatively mature software and many parts of it are very stable. Also, all the methodological developments presented in this thesis use a lot of algorithms which are shared among other post-processing tools, such as filtering or registration. This scenario suggests the necessity of integrating it along with other tools into a common post-processing platform. This platform should consist of a basic kernel, with all the common functions (DICOM support, visualization and image preparation) and several modules for each application that uses a particular set of algorithms. 



\section{Conclusions}

\section{Conclusions}

- The series of methodological developments and clinical applications presented in this thesis have demonstrated the feasibility of DCE-MRI PK analysis as a powerful medical tool for the study of angiogenesis in a noninvasive way.

- A pre-processing methodological development has focused on the data preparation. A temporal filter used to smooth enhancement curves without losing first-pass information was developed and implemented. It was based on the application of local regression with weighted linear least squares and first degree polynomials, corrected by the calculation of the curve gradients and the substitution of the first pass filtered values by the original data values. Although some image registration algorithms have been used through this thesis, these temporal filter has also been presented as a registration tool, as it has been shown that the main point of registration for PK analysis should be the extraction of accurate enhancement curves rather than accurate geometrical transforms.

- Regarding clinical applications, one main scope has been the study of different diseases. This work has focused on the study of cartilage degeneration, liver tumors (hepatocellular carcinoma) and grade-IV astrocytomas. In all the cases it has been demonstrated that PK parameters can effectively be used to characterize and separate diseased from normal tissues, even in relatively small numbers of patients. The obtaining of value ranges for normal and different stages of the disease is essential, in order to use these parameters in the clinical practice as early biomarkers of disease.

- On the evaluation of treatments, ovarian hyperstimulation syndrome (OHSS) treatment with cabergoline and osteoarthritic cartilage treatment 
with glucosamine sulfate have been studied. Both OHSS and osteoarthritis are diseases which have an important angiogenic component, so it was hypothesized and also demonstrated, that the PK parameters, especially $K^{\text {trans }}$, could be used to assess microvascular changes induced by these treatments.

- Two new developments have been carried out for data analysis and results presentation. First, the application of multivariate analysis techniques to the PK parametric images, which resulted in the generation of nosologic images. These images can directly offer information about the probability of disease for a certain voxel or region, which is the natural consequence of handling quantitative parametric maps. To obtain them, factorial and discriminant analysis were used. Second, the development of a new visualization tool for the improved assessment of regional cartilage damage, both of the knee and the ankle. This tool was based on the multiplanar projections on coronal (patelar cartilage), transversal (tibial and tibio-astragalin cartilage) and radial (femoral cartilage) planes. The histograms kurtosis and the skewness were introduced as statistical measures of region heterogeneities.

- Another main methodological development has focused on the analysis of the arterial vascularization in hepatocellular carcinomas. It has been demonstrated that, in comparison to other established PK parameters such as $K^{\text {trans }}$ and $v_{p}$, the newly developed arterial index (AI) offers a different type of information considering the enhancement curve shape and scale. The AI assesses the degree of similarity between tissues and a reference AIF, like the aorta. The calculation of the AI was based on the separation of the first pass and washout phases of the enhancement curves and the fitting of the first pass curve to a gamma probability density function. This new index complements the generalized PK analysis. 
- The reproducibility of both the image acquisition and the image analysis methodology has also been properly assessed in all the developments and clinical applications. Even a study comparing magnetic field intensity (1.5 $\mathrm{T}$ vs. 3.0 T) was also carried out in order to evaluate the variability of the results.

- A software tool for the application of PK models in clinical routine has been implemented in the Radiology Departments of Hospital Quirón Valencia and Hospital Universitari Dr. Peset of Valencia. 



\section{References}

[1] O'Connor JPB, Jackson A, Asselin MC et al. Quantitative imaging biomarkers in the clinical development of targeted therapeutics: current and future perspectives. Lancet Oncol 2008;9:766-776.

[2] Leach MO, Brindle KM, Evelhoch JL et al. The assessment of antiangiogenic and antivascular therapies in early-stage clinical trials using magnetic resonance imaging: issues and recommendations. Br J Cancer 2005;92:1599-1610.

[3] Collins DJ, Padhani AR. Dynamic magnetic resonance imaging of tumor perfusion. Approaches and biomedical challenges. IEEE Eng Med Biol Mag 2004;23:65-83.

[4] Tofts PS, Brix G, Buckley DL et al. Estimating kinetic parameters from dynamic contrast-enhanced T1-weighted MRI of a diffusable tracer: standardized quantities and symbols. J Magn Reson Imaging 1999;10:223-232.

[5] Hayes C, Padhani R, Leach MO. Assessing changes in tumour vascular function using dynamic contrast-enhanced magnetic resonance imaging. NMR Biomed 2002;15:154-163.

[6] Taylor JS, Reddick WE. Evolution from empirical dynamic contrast-enhanced magnetic resonance imaging to pharmacokinetic MRI. Adv Drug Deliv Rev 2000;41:91-110.

[7] Materne R, Smith AM, Peeters F et al. Assessment of hepatic perfusion parameters with dynamic MRI. Magn Reson Med 2002;47:135-142.

[8] D'Arcy JA, Collins DJ, Padhani AR et al. Magnetic resonance imaging workbench: analysis and visualization of dynamic contrast-enhanced MR imaging data. Radiographics 2006;26:621-632.

[9] Michoux N, Vallée JP, Pechère-Bertschi A et al. Analysis of contrast-enhanced MR images to assess renal function. Magn Reson Mater Phy 2006;19:167-179.

[10] Horsfield MA, Morgan B. Algorithms for calculation of kinetic parameters from T1-weighted dynamic contrast-enhanced magnetic resonante imaging. J Magn Reson Imaging 2004;20:723-729.

[11] Jackson A, Jayson GC, Li KL et al. Reproducibility of quantitative dynamic contrast-enhanced MRI in newly presenting glioma. Br J Radiol 2003;76:153-162. 
[12] Roberts C, Issa B, Stone A et al. Comparative study into the robustness of compartmental modeling and model-free analysis in DCE-MRI studies. J Magn Reson Imaging 2006; 23:554-563.

[13] Van Beers B, Leconte I, Materne R et al. Hepatic perfusion parameters in chronic liver disease: dynamic CT measurements correlated with disease severity. AJR Am J Roentgenol 2001;176:667-673.

[14] Padhani AR, Hayes C, Landau S et al. Reproducibility of quantitative dynamic MRI of normal human tissues. NMR Biomed 2002;15:143-153.

[15] Li KL, Zhu XP, Waterton J et al. Improved 3D quantitative mapping of blood volume and endothelial permeability in brain tumors. J Magn Reson Imaging 2000;12:347-357.

[16] Materne R, Van Beers BE, Smith AM et al. Non-invasive quantification of liver perfusion with dynamic computed tompography and a dual-input onecompartmental model. Clin Sci (Lond) 2000;99:517-525.

[17] Workie DW, Dardzinski BJ. Quantifying dynamic contrast-enhanced MRI of the knee in children with juvenile rheumatoid arthritis using an arterial input function (AIF) extracted from popliteal artery enhancement and the effect of the choice of the AIF on the kinetic parameters. Magn Reson Med 2005;54:560-568.

[18] Armitage P, Behrenbruch C, Brady $\mathrm{M}$ et al. Extracting and visualizing physiological parameters using dynamic contrast-enhanced magnetic resonance imaging of the breast. Med Image Anal 2005;9:315-329.

[19] Harrer JU, Parker GJM, Haroon HA et al. Comparative study of methods for determining vascular permeability and blood volume in human gliomas. J Magn Reson Imaging 2004;20:748-757.

[20] Haroon HA, Buckley DL, Patankar TA et al. A comparison of $\mathrm{K}^{\text {trans }}$ measurements obtained with conventional and first pass pharmacokinetic models in human gliomas. J Magn Reson Imaging 2004;19:527-536.

[21] Rijpkema M, Kaanders JH, Joosten FB et al. Method for quantitative mapping of dynamic MRI contrast agent uptake in human tumors. J Magn Reson Imaging 2001;14:457-463.

[22] Padhani AR. Dynamic contrast-enhanced MRI in clinical oncology: current status and futures directions. J Magn Reson Imaging 2002;16:407-422. 
[23] Hanahan D, Weinberg RA. The hallmarks of cancer. Cell 2000;100:57-70.

[24] Geiger TR, Peeper DS. Metastasis mechanisms. Biochim Biophys Acta 2009;1796:293:308.

[25] Gimbrone MA, Leapman SB, Cotran RS et al. Tumor dormancy in vivo by prevention of neovascularization. J Exp Med 1972;136:261-276.

[26] Folkman J. In: DeVita VT Jr, Hellman S, Rosenberg SA, eds. Cancer: Principles \& Practice of Oncology. Vol 2. $7^{\text {th }}$ ed. Philadelphia, PA: Lippincott Williams \& Wilkins; 2005:2865-2882.

[27] Ferrara N. Vascular endothelial growth factor: basic science and clinical progress. Endocr Rev 2004;25:581-611.

[28] Ellis LM, Hicklin DJ. VEGF-targeted therapy: mechanisms of anti-tumour activity. Nat Rev Cancer 2008;8:579-591.

[29] Furuya M, Yonemitsu Y, Aoki I. Angiogenesis: complexity of tumor vasculature and microenvironment. Curr Pharm Des 2009;15:1854-1867.

[30] Munn LL. Aberrant vascular architecture in tumors and its importance in drug-based therapies. Drug Discov Today 2003;8:396-403.

[31] Hoeben A, Landuyt B, Highley MS et al. Vascular endothelial growth factor and angiogenesis. Pharmacol Rev 2004;56:549-580.

[32] Delvingne A, Rozenberg S. Epidemiology and prevention of ovarian hyperstimulation syndrome (OHSS): a review. Hum Reprod Update 2002;8:559577.

[33] Cluroe AD, Synek BJ. A fatal case of ovarian hyperstimulation syndrome with cerebral infarction. Pathology 1995;27:344-346.

[34] Pellicer A, Albert C, Mercader A et al. The pathogenesis of ovarian hyperstimulation syndrome: in vivo studies investigating the role of interleukin$1 \beta$, interleukin-6, and vascular endothelial growth factor. Fertil Steril 1999;71:482489.

[35] Golan A, Rone-el R, Herman A et al. Ovarian hyperstimulation syndrome: an update review. Obstet Gynecol Surv 1989;44:430-440. 
[36] Gold GE, McCauley TR, Gray ML et al. What's new in cartilage? Radiographics 2003;23:1227-1242.

[37] Waldschmidt JG, Riling RJ, Kajdacsy-Balla AA et al. In vitro and in vivo MR imaging of hyaline cartilage: zonal anatomy, imaging pitfalls, and pathologic conditions. Radiographics 1997;17:1387-1402.

[38] Outerbridge RE. The etiology of chondromalacia patellae. J Bone Joint Surg Br 1961;43:752-757.

[39] Martí-Bonmatí L, Montaner D, Sanfeliu M. Análisis de la perfusión del cartílago rotuliano mediante resonancia magnética dinámica: aplicación en sujetos con dolor anterior de origen rotuliano. Rev Clin Esp 1999;199:641-646.

[40] Enomoto $\mathrm{H}$, Inoki I, Komiya $\mathrm{K}$ et al. Vascular endothelial growth factor isoforms and their receptors are expressed in human osteoarthritic cartilage. Am J Pathol 2003;162:171-181.

[41] Pfander D, Körtje D, Zimmermann R et al. Vascular endothelial growth factor in articular cartilage of healthy and osteoarthritic human knee joints. Ann Rheum Dis 2001;60:1070-1073.

[42] Tanaka E, Aoyama J, Miyauchi M et al. Vascular endothelial growth factor plays an important autocrine/paracrine role in the progression of osteoarthritis. Histochem Cell Biol 2005;123:275-281.

[43] Smith JO, Oreffo ROC, Clarke NMP et al. Changes in the antioangiogenic properties of articular cartilage in osteoarthritis. J Orthop Sci 2003;8:849-857.

[44] Fenwick SA, Gregg PJ, Rooney P. Osteoarthritic cartilage loses its ability to remain avascular. Osteoarthritis Cartilage 1999;7:441-452.

[45] Honorati MC, Cattini L, Facchini A. IL-17, IL-1b and TNF-a stimulate VEGF production by dedifferentiated chondrocytes. Osteoarthritis Cartilage 2004;12:683-691.

[46] Murata M, Yudoh K, Masuko K. The potential role of vascular endothelial growth factor (VEGF) in cartilage. How the angiogenic factor could be involved in the pathogenesis of osteoarthritis? Osteoarthritis Cartilage 2008;16:279-286.

[47] Bonnet CS, Walsh DA. Osteoarthritis, angiogenesis and inflammation. Rheumatology 2005;44:7-16. 
[48] Brat DJ, Parisi JE, Kleinschmidt-DeMasters BK et al. Surgical neuropathology update. A review of changes introduced by the WHO classification of tumours of the central nervous system, $4^{\text {th }}$ edition. Arch Pathol Lab Med 2007;132:993-1007.

[49] Stupp R, Mason WP, van den Bent MJ et al. Radiotherapy plus concomitant and adjuvant temozolomide for glioblastoma. N Engl J Med 2005; 352:987-996.

[50] Cha S. Update on brain tumor imaging: from anatomy to phisiology. Am J Neuroradiol 2006;27:475-487

[51] Burger P. Malignant astrocytic neoplasms: classification, pathology, anatomy, and response to therapy. Semin Oncol 1986; 13:16-20.

[52] World Health Organization

(www.who.int/mediacentre/factsheets/fs297/en/). Last access: November 13, 2009.

[53] Medline Plus (www.nlm.nih.gov/medlineplus/ency/article/000280.htm). Last access: November 13, 2009

[54] Jacobs MA, Ibrahim TS, Ouwerkerk R. AAPM/RSNA physics tutorial for residents: MR imaging: brief overview and emerging applications. Radiographics 2007;27:1213-1229.

[55] Vlaardingerbroek MT, De Boer JA. Magnetic resonance imaging: theory and practice. Springer, Germany, 2002.

[56] Moratal D, Martí-Bonmatí L, Gili J. European Directive 2004/40/EC on workers' exposure to electromagnetic fields from MRI. Radiología 2009;51:30-37.

[57] Erlemann R, Sciuk J, Bosse A et al. Response of osteosarcoma and Ewing sarcoma to preoperative chemotherapy: assessment with dynamic and static MR imaging and skeletal scintigraphy. Radiology 1990;175:791-796.

[58] Kaiser WA, Zeitler E. MR imaging of the breast: fast imaging sequences with and without Gd-DTPA. Radiology 1989;170:681-686.

[59] Elemann R, Reiser MF, Peters PE et al. Musculoskeletal neoplasms: static and dynamic Gd-DTPA-enhanced MR imaging. Radiology 1989;171:767-773.

[60] Buckley DL, Mussurakis S, Horsman A. Effect of temporal resolution on the diagnostic efficacy of contrast-enhanced MRI in the conservatively treated breast. J Comput Assist Tomogr 1998;22:47-51. 
[61] Roberts TPL. Physiologic measurements by contrast-enhanced MR imaging: expectations and limitations. J Magn Reson Imaging 1997;7:82-90.

[62] Hittmair K, Gomiscek G, Langenberger K et al. Method for quantitative assessment of contrast agent uptake in dynamic contrast-enhanced MRI. Magn Reson Med 1994;31:567-571.

[63] Brix G, Semmler G, Port R et al. Pharmacokinetic parameters in CNS GdDTPA enhanced MR imaging. J Comput Assist Tomogr 1991;15:621-628.

[64] Donahue KM, Burstein D, Manning WJ et al. Studies of Gd-DTPA relaxivity and proton exchange rates in tissue. Magn Reson Med 1994;32:66-76.

[65] Bloembergen N, Morgan LO. Proton relaxation times in paramagnetic solutions. Effects of electron spin relaxation. J Chem Phys 1961;34:842-850.

[66] Luz Z, Meiboom S. Proton relaxation in dilute solutions of cobalt (II) and nickel (II) ions in methanol and the rate of methanol exchange of the solvation sphere. J Chem Phys 1964;40:2686-2692.

[67] Tofts PS. Modeling tracer kinetics in dynamic Gd-DTPA MR imaging. J Magn Reson Imaging 1997;7:91-101.

[68] Kety SS. The theory and applications of the exchange of inert gas at the lungs and tissues. Pharmacol Rev 1951;3:1-41.

[69] St. Lawrence KS, Lee TY. An adiabatic approximation to the tissue homogeneity model for water exchange in the brain: I. Theoretical derivation. J Cereb Blood Flow Metab 1998;18:1365-1377.

[70] Li KL, Zhu XP, Waterton J et al. Improved 3D quantitative mapping of blood volume and endothelial permeability in brain tumors. J Magn Reson Imaging 2000;12:347-357.

[71] Patlak CS, Blasberg RG, Fenstermacher JD. Graphical evaluation of blood-tobrain barrier transfer constants from multiple time-uptake data. J Cereb Blood Flow Metab 1983;3:1-7.

[72] Weinmann HJ, Laniado M, Mützel W. Pharmacokinetics of GdDTPA/dimeglumine after intravenous injection into healthy volunteers. Physiol Chem Phys Med NMR 1984;16:167-172. 
[73] Oshida K, Nagashima T, Ueda T et al. Pharmacokinetic analysis of ductal carcinoma in situ of the breast using dynamic MR mammography. Eur Radiol 2005;15:1353-1360.

[74] Van Beers BE, Leconte I, Materne R et al. Hepatic perfusion parameters in chronic liver disease: dynamic CT measurements correlated with disease severity. AJR Am J Roentgenol 2001;176:667-673.

[75] Annet L, Hermoye L, Peeters F et al. Glomerular filtration rate: assessment with dynamic contrast-enhanced MRI and a cortical-compartment model in the rabbit kidney. J Magn Reson Imaging 2004;20:843-849.

[76] Hawighorst $\mathrm{H}$, Libicher M, Knopp MV et al. Evaluation of angiogenesis and perfusion of bone marrow lesions: role of semiquantitative and quantitative dynamic MRI. J Magn Reson Imaging 1999;10:286-294.

[77] Ocak I, Bernardo M, Metzger G et al. Dynamic contrast-enhanced MRI of prostate cancer at $3 \mathrm{~T}$ : a study of pharmacokinetic parameters. AJR Am J Roentgenol 2007;189:192-201.

[78] Singh A, Haris M, Rathore D et al. Quantification of physiological and hemodynamic indices using T1 dynamic contrast-enhanced MRI in intracranial mass lesions. J Magn Reson Imaging 2007;26:871-880.

[79] Álvarez C, Martí-Bonmatí L, Novella-Maestre E et al. Dopamine against cabergoline reduces hemoconcentration and ascites in hyperstimulated women undergoing assisted reproduction. J Clin Endocrinol Metab 2007;92:2931-2937.

[80] Orton MR, d'Arcy JA, Walker-Samuel S et al. Computationally efficient vascular input function models for quantitative kinetic modelling using DCEMRI. Phys Med Biol 2008;53:1225-1239.

[81] Ródenas J. Obtención automática de la función de entrada arterial en imágenes de resonancia magnética de perfusión de ovarios mediante análisis de componentes independientes. Tesis doctoral. Universitat Politècnica de València, 2008 .

[82] Wang L, Schweitzer ME, Padua A et al. Rapid 3D-T1 mapping of cartilage with variable flip angle and parallel imaging at 3.0 T. J Magn Reson Imaging 2008;27:154-161. 
[83] Ahearn TS, Staff RT, Redpath TW et al. The use of the Levenberg-Marquardt curve-fitting algorithm in pharmacokinetic modelling of DCE-MRI data. Phys Med Biol 2005;50:85-92.

[84] den Dekker AJ, Sijbers J. Estimation of signal and noise parameters from MR data. In: Advanced image processing in magnetic resonance imaging. Eds: Landini L, Positano V, Santarelli MF. CRC Press, Boca Raton, 2005.

[85] Ahn CB, Song YC, Park DJ. Adaptive template filtering for signal-to-noise ratio enhancement in magnetic resonance imaging. IEEE Trans Med Imaging 1999;18:549-556.

[86] $\mathrm{Xu} \mathrm{Y,} \mathrm{Weaver} \mathrm{JB,} \mathrm{Healy} \mathrm{DM} \mathrm{et} \mathrm{al.} \mathrm{Wavelet} \mathrm{transform} \mathrm{domain} \mathrm{filters:} \mathrm{a}$ spatially selective noise filtration technique. IEEE Trans Image Process 1994;3:747-757.

[87] Manjón JV, Carbonell-Caballero J, Lull JJ et al. MRI denoising using nonlocal means. Med Image Anal 2008;12:514-523.

[88] Perona P, Malik J. Scale space and edge detection using anisotropic diffusion. IEEE Trans Pattern Anal Machine Intell 1990;12:629-639.

[89] Sijbers J, den Dekker AJ, Verhoye AM et al. Adaptive anisotropic noise filtering for magnitude MR data. Magn Reson Imaging 1999;17:1533-1539.

[90] Styner M, Brechbuhler C, Szekely G et al. Parametric estimate of intensity inhomogeneities applied to MRI. IEEE Trans Med Imaging 2000;19:153-165.

[91] Pruessmann KP, Weiger M, Scheidegger MB et al. SENSE: sensitivity encoding for fast MRI. Magn Reson Med 1999;42:952-962.

[92] Cleveland WS. Robust locally weighted regression and smoothing scatterplots. J Am Stat Assoc 1979;74:829-836.

[93] Savitzky A, Golay MJE. Smoothing and differentiation of data by simplified least squares procedures. Anal Chem 1964;36:1627-1639.

[94] Ivancevic MK, Kwee TC, Takahara T et al. Diffusion-weighted MR imaging of the liver at 3.0 Tesla using tracking only navigator echo (TRON): a feasibility study. J Magn Reson Imaging 2009;30:1027-1033.

[95] Maintz JBA, Viergever MA. A survey of medical image registration. Med Image Anal 1998;2:1-36. 
[96] Crum WR, Hartkens T, Hill DLG. Non-rigid image registration: theory and practice. Br J Radiol 2004;77:140-153.

[97] Maes F, Collignon A, Vandermeulen D et al. Multimodality image registration by maximization of mutual information. IEEE Trans Med Imaging 1997;16:187-198.

[98] Zhang Y, Zou P, Mulhern RK et al. Brain structural abnormalities in survivors of pediatric posterior fossa brain tumors: a voxel-based morphometry study using free-form deformation. Neuroimage 2008;42:218-229.

[99] Ashburner J, Friston KJ. Nonlinear spatial normalization using basis functions. Hum Brain Mapp 1999;7:254-266.

[100] Sanz-Requena R, Martí-Bonmatí L, Alvarez C et al. Resonancia magnética en la respuesta al tratamiento de hiperestimulación ovárica: comparación de modelos farmacocinéticos. Radiología 2009;51:176-182.

[101] Sanz R, Martí-Bonmatí L, Rodrigo JL et al. MR pharmacokinetic modeling of the patellar cartilage differentiates normal from pathological conditions. J Magn Reson Imaging 2008;27:171-177.

[102] Martí-Bonmatí L, Sanz-Requena R, Rodrigo JL et al. Glucosamine sulfate effect on the degenerated patellar cartilage: preliminary findings by pharmacokinetic magnetic resonance modeling. Eur Radiol 2009;19:1512-1518.

[103] Martí-Bonmatí L, Sanz-Requena R, Alberich-Bayarri A. Pharmacokinetic MR analysis of the cartilage is influenced by field strength. Eur J Radiol 2008;67:448-452.

[104] Prieto L, Lamarca R, Casado A. La evaluación de la fiabilidad en las observaciones clínicas: el coeficiente de correlación intraclase. Med Clin (Barc) 1998;110:142-145.

[105] Gold GE, Han E, Stainsby J et al. Musculoskeletal MRI at 3.0T: relaxation times and image contrast. AJR Am J Roentgenol 2004;183:343-351.

[106] Buckley DL. Uncertainty in the analysis of tracer kinetics using dynamic contrast-enhanced T1-weighted MRI. Magn Reson Med 2002;47:601-606.

[107] Otsu N. A threshold selection method from gray-level histograms. IEEE Trans Syst Man Cybern 1979;9:62-66. 
[108] Liow RY, Walker K, Wajid MA et al. The reliability of the American Knee Society Score. Acta Orthop Scand 2000;71:603-608.

[109] Chiang IC, Kuo YT, Lu CY et al. Distinction between high-grade gliomas and solitary metastases using peritumoral 3T magnetic resonance spectroscopy, diffusion and perfusion imaging. Neuroradiology 2004;46:619-627.

[110] Ostergaard L, Weisskoff RM, Chesler DA et al. High resolution measurement of cerebral blood flow using intravascular tracer bolus passages. Part I: mathematical approach and statistical analysis. Magn Reson Med 1996;36:715-725.

[111] Emblem K, Nedregaard B, Nome T et al. Glioma grading using histogram analysis of blood volume heterogeneity from MR-derived cerebral blood volume maps. Radiology 2008;247:808-817.

[112] Zur Y, Stokar S, Bendel P. An analysis of fast imaging sequences with steady-state magnetization refocusing. Magn Reson Med 1988;6:175-193.

[113] Stammberger T, Eckstein F, Englmeier $\mathrm{KH}$ et al. Determination of 3D cartilage thickness data from MR imaging: computational method and reproducibility in the living. Magn Reson Med 1999;41:529-536.

[114] Lin PC, Reiter DA, Spencer RG. Classification of degraded cartilage through multiparametric MRI analysis. J Magn Reson 2009; epub ahead of print.

[115] Carlos RC, Kim HM, Hussain HK et al. Developing a prediction rule to assess hepatic malignancy in patients with cirrhosis. AJR Am J Roentgenol 2003;180:893-900.

[116] Geladi P. Sampling and local models for multivariate image analysis. Mikrochim Acta 1995;120:211-230.

[117] Martinez AM, Kak AC. PCA versus LDA. IEEE Trans Pattern Anal Mach Intell 2001;23:228-233.

[118] Imhof H, Sulzbacher I, Grampp S et al. Subchondral bone and cartilage disease: a rediscovered functional unit. Invest Radiol 2000;35:581-588.

[119] Slater RNS, Spencer JD, Churchill MA et al. Observations on the intrinsic blood supply to the human patella: disruption correlated with articular surface degeneration. J R Soc Med 1991;84:606-607. 
[120] Scharf J, Kemmling A, Hess T et al. Assessment of hepatic perfusion in transplanted livers by pharmacokinetic analysis of dynamic magnetic resonance measurements. Invest Radiol 2007;42:224-229.

[121] Bulakbasi N, Kocaoglu M, Farzaliyev A et al. Assessment of diagnostic accuracy of perfusion MR imaging in primary and metastatic solitary malignant brain tumors. AJNR Am J Neuroradiol 2005;26:2187-219.

[122] Law M, Young R, Babb J et al. Comparing perfusion metrics obtained from a single compartment versus pharmacokinetic modeling methods using dynamic susceptibility contrast-enhanced perfusion MR imaging with glioma grade. AJNR Am J Neuroradiol 2006;21:1975-1982.

[123] Rose CJ, Mills SJ, O'Connor JP et al. Quantifying spatial heterogeneity in dynamic contrast-enhanced MRI parameter maps. Magn Reson Med 2009;62:488499.

[124] Cheng HLM. Investigation and optimization of parameter accuracy in dynamic contrast-enhanced MRI. J Magn Reson Imaging 2008;28:736-743.

[125] Singh A, Rathore RKS, Haris $M$ et al. Improved bolus arrival time and arterial input function estimation for tracer kinetics analysis in DCE-MRI. J Magn Reson Imaging 2009;29:166-176.

[126] Yang C, Karczmar GS, Medved M et al. Estimating the arterial input function using two reference tissues in dynamic contrast-enhanced MRI studies: fundamental concepts and simulations. Magn Reson Med 2004;52:1110-1117.

[127] Parker GJM, Roberts C, Macdonald A et al. Experimentally-derived functional form for a population-averaged high-temporal-resolution arterial input function for dynamic contrast-enhanced MRI. Magn Reson Med 2006;56:993-1000.

[128] Yang X, Liang J, Heverhagen JT et al. Improving the pharmacokinetic parameter measurement in dynamic contrast-enhanced MRI by use of the arterial input function: theory and clinical application. Magn Reson Med 2008;59:14481456.

[129] Hyvärinen A, Oja E. Independent component analysis: algorithms and application. Neural Networks 2000;13:411-430. 
[130] Hackstein N, Heckrodt J, Rau WS. Measurement of single-kidney glomerular filtration rate using a contrast-enhanced dynamic gradient-echo sequence and the Rutland-Patlak plot technique. J Magn Reson Imaging 2003;18:714-725.

[131] Le Bihan D, Delannoy DJ, Levin RL. Temperature mapping with MR imaging of molecular diffusion: application to hyperthermia. Radiology $1988 ; 171: 853-857$. 


\section{Publications}

\section{International journals}

- Álvarez C, Marti-Bonmati L, Novella-Maestre E, Sanz R, Gomez R, Fernandez-Sanchez M, Simon C, Pellicer A. Dopamine agonist cabergoline reduces hemoconcentration and ascites in hyperstimulated women undergoing assisted reproduction. J Clin Endocrinol Metab 2007;92:2931-7.

- Sanz R, Marti-Bonmati L, Rodrigo JL, Moratal D. MR pharmacokinetic modeling of the patellar cartilage differentiates normal from pathological conditions. J Magn Reson Imaging 2008;27:171-7.

- Marti-Bonmati L, Sanz-Requena R, Alberich-Bayarri A. Pharmacokinetic MR analysis is influenced by field strength. Eur J Radiol 2008;67:448-452.

- Martí-Bonmatí L, Sanz-Requena R, Rodrigo JL, Alberich-Bayarri A, Carot JM. Glucosamine sulfate effect on the degenerated patellar cartilage: preliminary findings by pharmacokinetic magnetic resonance modeling. Eur Radiol 2009;19:1512-1518.

- Martí-Bonmatí L, Alberich-Bayarri A, Sanz-Requena R, Sánchez-González J. State of the art in liver imaging - MR diffusion/perfusion. Controversies and Consensus in Imaging and Intervention, 2010.

- Sanz-Requena R, Martí-Bonmatí L, Pellicer B, Alberich-Bayarri A, GarcíaMartí G, Moratal D. Arterial index: a new imaging biomarker to assess vascularization in DCE-MR images of hepatocellular carcinomas. [submitted to Magn Res Med].

\section{National journals}

- Sanz Requena R, Martí-Bonmatí L, Álvarez C, García G, Pellicer A, Alberich Bayarri A, Moratal D. Resonancia magnética en la respuesta al tratamiento del síndrome de hiperestimulación ovárica: comparación de modelos farmacocinéticos. Radiología 2009; 51:176-182.

- Martí-Bonmatí L, Sanz R, Alberich A, Belloch E. Nuevas técnicas de imagen en la valoración del cartílago articular. Reumatol Clin 2009; epub.

- Martí-Bonmatí L, Sanz-Requena R, Rodrigo JL. La neovascularización del cartílago articular es un excelente biomarcador de la degeneración y permite monitorizar el efecto del tratamiento. Salud Rural 2009;26:37-44.

- Sanz-Requena R, Martí-Bonmatí L, Hervás V, Vega M, Alberich-Bayarri A, García-Martí G, Carot JM. Modificación del tiempo de relajación longitudinal 
(T1) como biomarcador de la degeneración del cartílago patelar. Radiología 2010; in press.

- Revert Ventura AJ, Sanz-Requena R, Martí-Bonmatí L, Jornet J, Piquer J, Cremades A, Carot JM. Análisis nosológico con parámetros de perfusión tisular de RM obtenidos mediante los modelos monocompartimental y farmacocinético en glioblastomas cerebrales. Radiología 2010; in press.

\section{Book chapters}

- Martí-Bonmatí L, Sanz Requena R, Moratal Pérez D. Cuantificación de la captación en resonancia magnética. In: Monografía SERAM Medios de contraste en radiología. Editores: Martí-Bonmatí L, Pallardó Calatayud Y. Editorial Médica Panamericana S.A. Madrid, ISBN 978-84-9835-161-3, 2008.

- Martí-Bonmatí L, Sanz Requena R, Alberich Bayarri A. Nuevos horizontes en la imagen en oncología. In: Actualizaciones SERAM Imagen en oncología. Editores: Pallardó Calatayud Y, Revert Ventura AJ, Cervera Deval J. Editorial Médica Panamericana S.A. Madrid, ISBN 978-84-9835-162-0, 2008.

- Martí-Bonmatí L, Sanz Requena R, García Martí G, Flórez YN, Alberich Bayarri A. Modelado de sistemas dinámicos en imagen médica. In: Ingeniería Biomédica, Imágenes Médicas. Sistemas Dinámicos. Servicio de Publicaciones de la Universidad de Castilla-La Mancha, 2008.

- Sanz Requena R, Martí-Bonmatí L, Alberich Bayarri A, García Martí G, Flórez Ordóñez N. Cuantificación de estudios dinámicos y compartimentales. In: Ingeniería Biomédica, Nuevas técnicas de procesado de imágenes médicas. Servicio de Publicaciones de la Universidad de Castilla-La Mancha, 2009.

- Martí-Bonmatí L, Sanz Requena R, García Martí G, Alberich Bayarri A. Biomarcadores de imagen: su importancia en la imagen médica. In: Ingeniería Biomédica, Nuevas técnicas de procesado de imágenes médicas. Servicio de Publicaciones de la Universidad de Castilla-La Mancha, 2009.

\section{International congresses}

- Rieta JJ, Moratal D, Martí-Bonmatí L, Molina-Mínguez R, Valles-Lluch A, Sanz R. ICA for ovary tissue classification of perfusion magnetic resonance images. 29th IEEE EMBS Annual International Conference, Lyon, France, 2007.

- Martí-Bonmatí L, Sanz R. MRI pharmacokinetic modelling of treatment effects in patients with ovarian hyperestimulation syndrome. European Congress of Radiology, Vienna, Austria, 2008. 
- Martí-Bonmatí L, Sanz R. Pharmacokinetic analysis of the patellar cartilage in DCE-MRI: Comparison between 1.5 and 3 Tesla. European Congress of Radiology, Vienna, Austria, 2008.

- Sanz-Requena R, Rodenas J, Martí-Bonmatí L, Alberich-Bayarri A, Pellicer A. Influence of the arterial input function selection in MR pharmacokinetic modeling of ovarian hyperestimulation syndrome: a comparison of individual, population averaged and ICA-extracted functions. 25th Annual Meeting ESMRMB, Valencia, Spain, 2008.

- Ródenas-García J, Sanz-Requena R, Martí-Bonmatí L, Rieta-Ibáñez J. Accurate arterial input function extraction via independent component analysis for pharmacokinetic evaluation of ovaries. 25th Annual Meeting ESMRMB, Valencia, Spain, 2008.

- Ródenas-García J, Sanz-Requena R, Martí-Bonmatí L, García-Martí G, RietaIbáñez J. Independent component analysis of DCE-MR images of hepatocellular carcinoma for pharmacokinetic analysis. 25th Annual Meeting ESMRMB, Valencia, Spain, 2008.

- Sánchez-González J, Martí-Bonmatí L, Alberich-Bayarri A, García-Martí G, Sanz-Requena R. Variance of the cartilage T1 estimation by 3D spoiled gradient echo sequences with variable flip angle. 25th Annual Meeting ESMRMB, Valencia, Spain, 2008.

- Sanz-Requena R, Martí-Bonmatí L, Barroso D, García-Martí G. MR pharmacokinetic modeling of liver in cirrhotic and non-cirrhotic patients: analysis of pharmacokinetic parameters and liver heterogeneity. 25th Annual Meeting ESMRMB, Valencia, Spain, 2008.

- Sanz-Requena R, Martí-Bonmatí L, Barroso D, García-Martí G. MR pharmacokinetic modeling of liver in hepatocellular carcinoma and focal nodular hyperplasia patients: analysis of pharmacokinetic parameters and lesion heterogeneity. 25th Annual Meeting ESMRMB, Valencia, Spain, 2008.

- Sanz Requena R, Martí-Bonmatí L. Assessment of heterogeneities in hepatocarcinoma liver lesions by pharmacokinetic MR modeling and histogram analysis. 20th Annual Meeting of the ESGAR, Valencia, Spain, 2008.

- Sanz-Requena R, Revert A, Martí-Bonmatí L, Alberich-Bayarri A, GarcíaMartí G. Correlation between pharmacokinetic parameters, blood flow, blood volume and initial area under curve in grade-4 astrocytomas. 26th Annual Meeting ESMRMB, Antalya, Turkey, 2009.

- Sanz-Requena R, Martí-Bonmatí L, Hervás-Briz V, Alberich-Bayarri A, García-Martí G. Pharmacokinetic modeling of femoropatellar cartilage in 3T DCE-MR. 26th Annual Meeting ESMRMB, Antalya, Turkey, 2009. 
- Martí-Bonmatí L, Sanz-Requena R, Alberich-Bayarri A, García-Martí G. Multivariate analysis in medical imaging. International Workshop on Multivariate Image Analysis, Valencia, Spain, 2009.

- Revert A, Sanz-Requena R, Martí-Bonmatí L, Carot J, García-Martí G. Discriminant analysis of grade-IV astrocytomas from dynamic T2* contrastenhanced MR imaging. European Congress of Radiology, March 4-8, Vienna, Austria, 2010.

- Sanz-Requena R, Martí-Bonmatí L, Pellicer B, Moratal D, Alberich-Bayarri A. Arterial vascularization index assessment of hepatocellular carcinoma: a new DCE-MR imaging biomarker. European Congress of Radiology, March 4-8, Vienna, Austria, 2010.

- Sanz-Requena R, Martí-Bonmatí L, Hervás V, Llombart R, Carot J. A new pixel-based discriminant analysis classification of the osteoarthritic ankle cartilage from pharmacokinetic modeling of DCE-MR images. European Congress of Radiology, March 4-8, Vienna, Austria, 2010.

- Sanz-Requena R, Martí-Bonmatí L, Pellicer B, Alberich-Bayarri A, GarcíaMartí G. Heterogeneity of the arterial contribution in hepatocellular carcinomas evaluated from pharmacokinetic DCE-MR images. 21 ${ }^{\text {st }}$ Annual Meeting ESGAR, June 2-5, Dresden, Germany, 2010.

\section{National congresses}

- Sanz R, Martí-Bonmatí L, Alberich-Bayarri A, Moratal D. Modelado farmacocinético del cartílago rotuliano mediante estudio dinámico de resonancia magnética de 3T. XXIX Congreso Nacional de la SERAM, Sevilla, España, 2008.

- Sanz R, Martí-Bonmatí L, Alberich-Bayarri A, Moratal D. La intensidad de campo influye significativamente en los valores de los parámetros farmacocinéticos del cartílago rotuliano calculados de imágenes obtenidas en equipos de resonancia de 1,5T y 3T. XXIX Congreso Nacional de la SERAM, Sevilla, España, 2008.

- Sanz R, Martí-Bonmatí L, Alberich-Bayarri A, Moratal D. Modelado farmacocinético del efecto de la cabergolina en el tratamiento de ovarios con síndrome de hiperestimulación. XXIX Congreso Nacional de la SERAM, Sevilla, España, 2008.

- Ródenas García J, Sanz Requena R, Martí-Bonmatí L, García Martí G, Rieta JJ. Segmentación automática de imágenes de perfusión de resonancia magnética de hígado mediante separación ciega de fuentes: aplicación al análisis farmacocinético del carcinoma hepatocelular. XXVI Congreso Anual de la Sociedad Española de Ingeniería Biomédica, Valladolid, Spain, 2008. 
- Ródenas García J, Sanz Requena R, Martí-Bonmatí L, Alberich-Bayarri A, Pellicer Martínez A, Rieta JJ. Influencia de la selección de la función de entrada arterial en el modelado farmacocinético del síndrome de hiperestimulación ovárica a partir de imágenes de resonancia magnética. XXVI Congreso Anual de la Sociedad Española de Ingeniería Biomédica, Valladolid, Spain, 2008.

- Revert Ventura AJ, Sanz-Requena R, Martí-Bonmatí L, Pallardó Calatayud Y, Mollá Olmos E, Gaspar Martínez C. Aplicación de funciones discriminantes para la clasificación de regiones en estudios cuantitativos de perfusión por RM de glioblastomas. XXX Congreso Nacional de la SERAM, 28-31 Mayo, A Coruña, Spain, 2010.

- Revert Ventura AJ, Sanz-Requena R, Martí-Bonmatí L, Pallardó Calatayud Y, Jornet Fayos J, Gaspar Martínez C. Estudio de la curtosis de los histogramas como biomarcador de la heterogeneidad de las regiones en estudios cuantitativos de perfusión por RM de los glioblastomas. XXX Congreso Nacional de la SERAM, 28-31 Mayo, A Coruña, Spain, 2010.

- Sanz-Requena R, Martí-Bonmatí L, Hervás Briz V, Llombart Ais R, Carot Sierra JM, Alberich Bayarri A. Utilidad del análisis discriminante en la clasificación por grado de degeneración del cartílago patelar a partir del modelado farmacocinético de la perfusión por RM. XXX Congreso Nacional de la SERAM, 28-31 Mayo, A Coruña, Spain, 2010.

- Sanz-Requena R, Martí-Bonmatí L, Pellicer de Gracia B, García Martí G. Separación y análisis cuantitativo de las fases de primer paso y lavado en estudios de perfusión de RM de carcinoma hepatocelular. XXX Congreso Nacional de la SERAM, 28-31 Mayo, A Coruña, Spain, 2010. 



\section{Appendix}

\section{Developed software}

All the applications presented in this thesis have been developed in Matlab R2006b (The Mathworks Inc., Natick, MA, USA). A set of tools and graphical user interfaces (GUIs) were built in order to test and apply all the methodologies. The basic workflow followed in all the cases was (figure A.1):

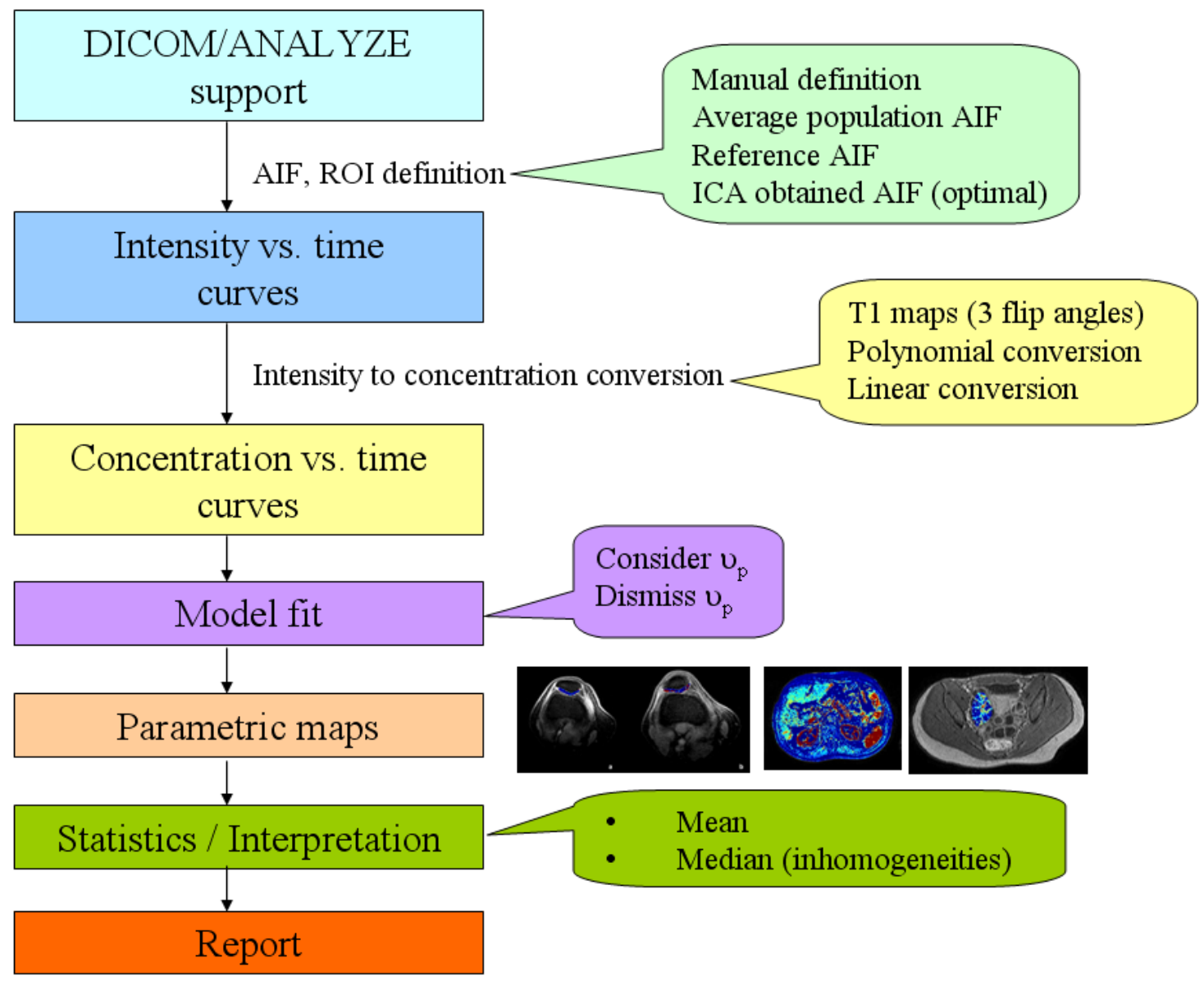

Figure A.1. PK analysis workflow. 


\section{Main GUI}

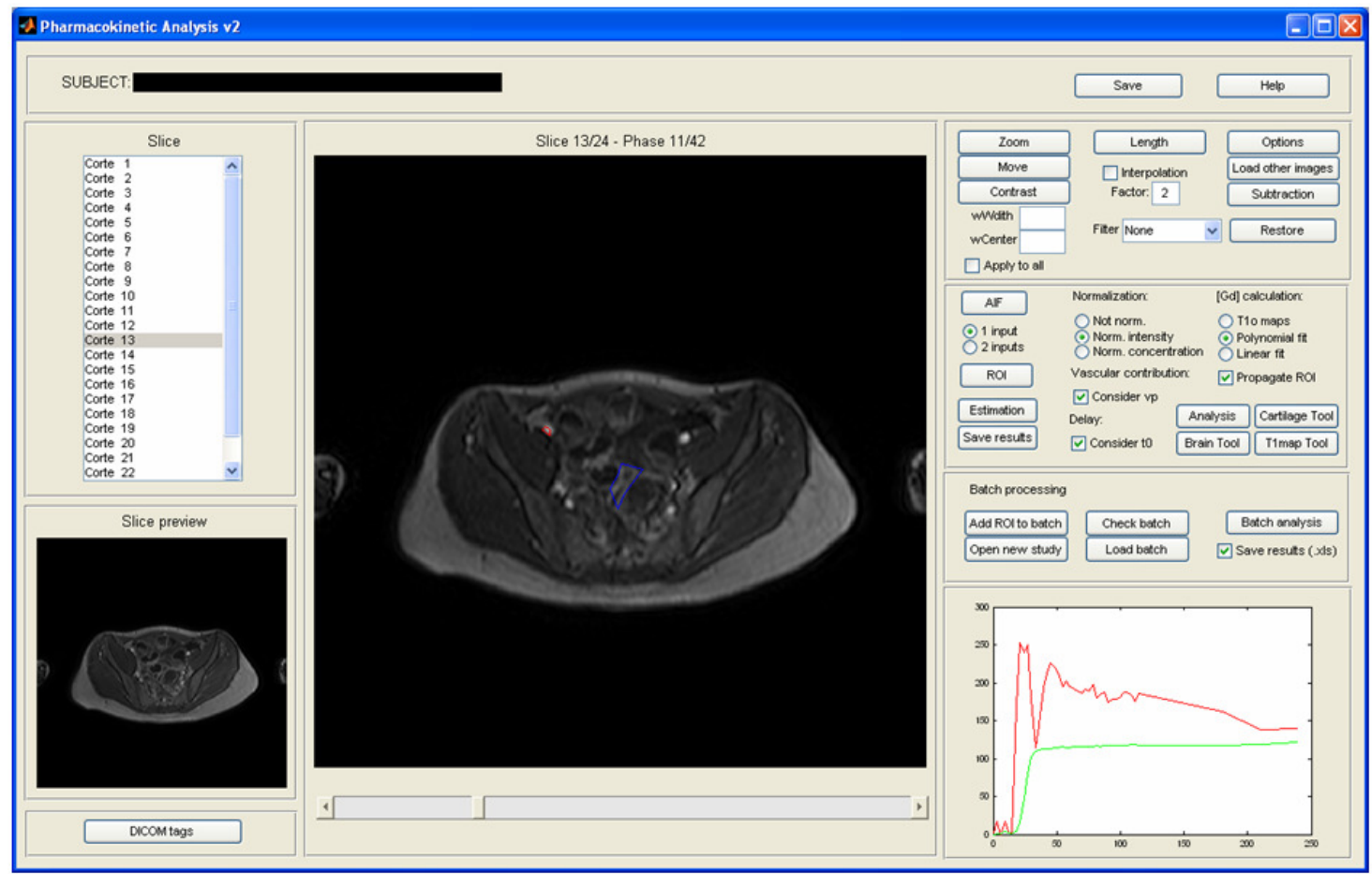

Figure A.2. Main GUI.

Characteristics:

- DICOM / Analyze support

- Image explorer for other images than DCE-MRI

- Visualization functions: zoom, move, contrast, length measures, subtraction, filters and plots

- PK modeling: 1 or 2 compartments, 1 or 2 inputs, normalization options and intensity to concentration conversion options

- Batch processing for multiple studies 


\section{Specific cartilage GUI}

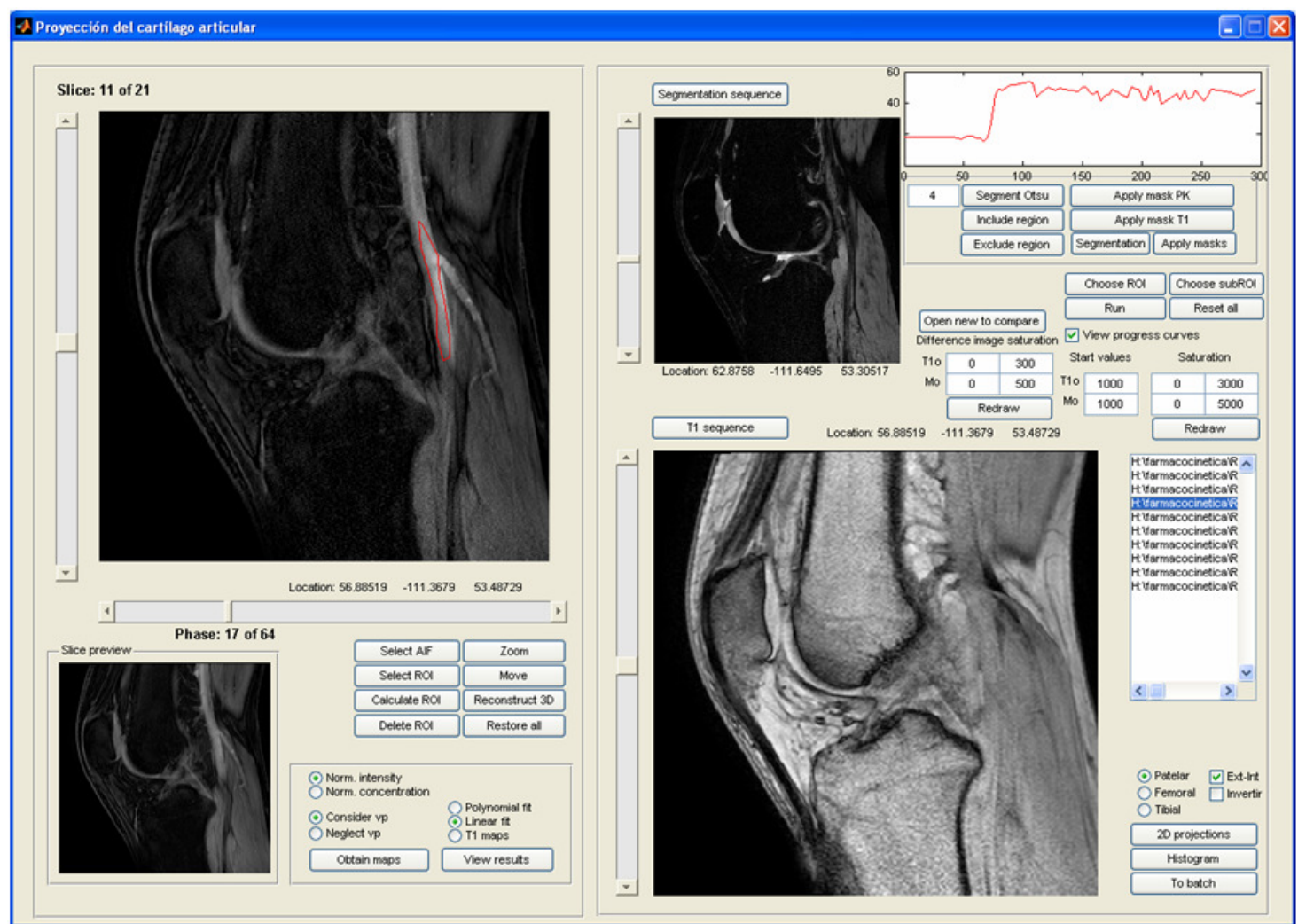

Figure A.3. Cartilage GUI.

Specific characteristics:

- Cartilage segmentation

- $\quad$ T1 mapping

- 2D projections 